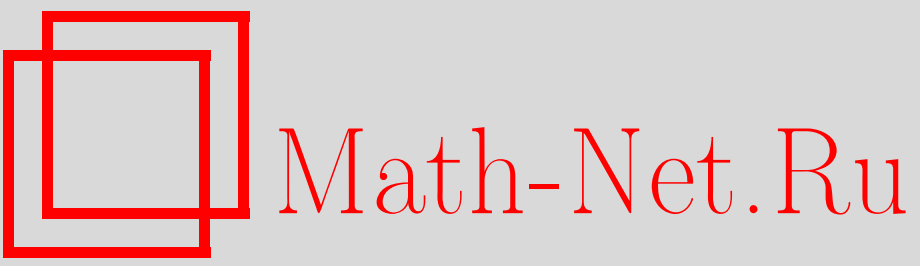

И. Я. Новиков, С. Б. Стечкин, Основы теории всплесков, УМН, 1998, том 53, выпуск 6, 53-128

DOI: https://doi.org/10.4213/rm89

Использование Общероссийского математического портала Math-Net.Ru подразумевает, что вы прочитали и согласны с пользовательским соглашением

http://www.mathnet.ru/rus/agreement

Параметры загрузки:

IP : 54.197 .217 .227

26 апреля 2023 г., 12:50:06 


\title{
ОСНОВЫ ТЕОРИИ ВСПЛЕСКОВ
}

\author{
И. Я. Новиков, С. Б. СтЕчкин
}

\section{СОДЕРЖАНИЕ}

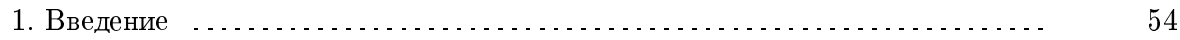

2. Обозначения и определения ................................. 54

3. Прототипы всплесков в работах Лузина и Калшдерона . . . . . . . . . . . . . . 57

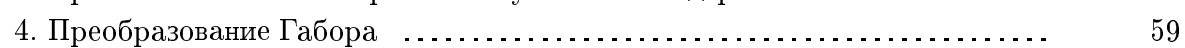

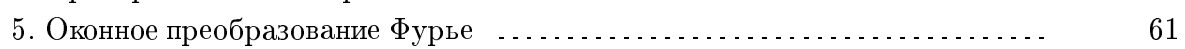

6. Интегральное всплесковое преобразование . . . . . . . . . . . . . . . . . . . . 62

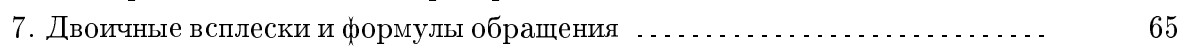

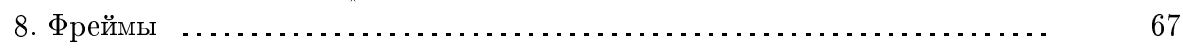

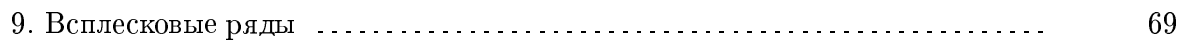



11. Кратномасштабньй анализ в $L^{2}(\mathbb{R})$. . . . . . . . . . . . . . . . . . . . . 74

12. Система Уиттакера-Шеннона-Котельникова ...................... . 80

13. Константы неопределенности ............................. 81

14. Всплески Мейера . . . . . . . . . . . . . . . . . . . . . . . . . . . . . . . 81

15. Всплески Лемари-Бэтла и Стремберга ... . . . . . . . . . . . . . . . . . . 83

16. Ортогональные всплески с компактньм носителем . . . . . . . . . . . . . . 86

17. Быстрые алгоритмы ... . . . . . . . . . . . . . . . . . . . . . . . . . . 89

18. Полуортогоналшные сплайн-всплески с компактньм носителем . . . . . . . 92

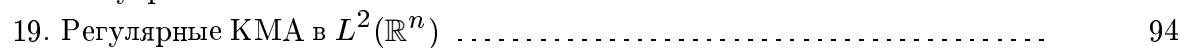

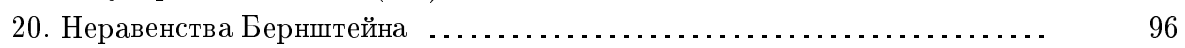

21. Регулярные КМА и полиномы ... . . . . . . . . . . . . . . . . . . . . . . . . 98

22. КМА в пространствах Соболева ................................ 104

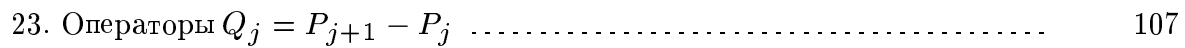

24. Пространства Бесова .................................... 111

25. Проекторы $P_{j}$ и псевдодифференциальные операторы $\ldots . . . . . . . . . .112$

26. Всплесковая характеризация пространств Гёльдера $\mathbb{C}^{s}$, Соболева $W_{2}^{s}$ и

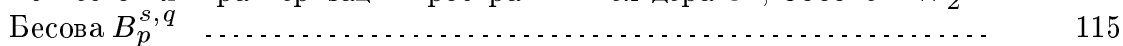

27. Всплесковая характеризация пространств $H^{1}\left(\mathbb{R}^{n}\right)$ и ВМО $\quad \ldots . . . . . .$.

28. Всплесковая характеризация пространств $L^{p}\left(\mathbb{R}^{n}\right)$ и $W_{p}^{s}\left(\mathbb{R}^{n}\right) \quad \ldots \ldots \ldots .$.

29. Периодические всплески . ................................ 123

Список литературы . . . . . . . . . . . . . . . . . . . . . . . . . . . . . . . 127

(С) и.я. Новиков, С. Б. Стечкин 1998 
1. Введение. Всплеском, в самом общем виде, назьвают определенную на числовой оси функцию $\psi$, имеюшую нулевое среднее и достаточно быстрое убывание на бесконечности. Термин "всплеск" предложен К.И. Осколковым в качестве эквивалента английского термина wavelet (фp. - ondelette), что буквально переводится как маленькая (имеется в виду продолжительность) волна, волночка. Термин "всплеск" лучше отражает суть дела, так как упомянутые выше свойства означают, что функция $\psi$ представляет собой затухающее колебание. Всплески используются или в качестве ядра интегрального преобразования

$$
\left(W_{\psi} f\right)(a, b)=\frac{1}{\sqrt{a}} \int_{\mathbb{R}} f(t) \psi\left(\frac{t-b}{a}\right) d t, \quad a, b \in \mathbb{R}, \quad a>0
$$

или в качестве генерирующей функции для построения базиса при помощи дилатаций, т.е. сжатий с сохранением нормы в $L^{2}(\mathbb{R}): \psi_{j}(t):=\psi_{j 0}(t):=2^{j / 2} \psi\left(2^{j} t\right), j \in \mathbb{Z}$, и сдвигов $\psi_{j k}(t):=\psi_{j}\left(t-k 2^{-j}\right)=2^{j / 2} \psi\left(2^{j} t-k\right), k \in \mathbb{Z}$.

Теория всплесков лежит на пересечении чистой математики, вычислительной математики, преобразования сигналов и изображений. Всплесковьй анализ находит все более широкое применение в различных областях науки, так как он дает более подробную информацию о сигнале, изображении или операторе, чем стандартньй анализ Фурье. Интегральное всплесковое преобразование дает одновременно локальную информацию о функции и о ее преобразовании Фурье, причем для анализа высокочастотных составляющих функции - локализация более сильная (для повышения точности), а для низкочастотных - локализация более слабая (для получения полной информации). Всплесковые ряды очень удобны для приближенных вычислений, поскольку количество операций, необходимых для вычисления коэффициентов разложения, так же как и количество операций для восстановления функции по ее всплесковым коэффициентам, пропорционально количеству отсчетов функции. Перечисленные особенности всплесков делают их очень популярными в самых различных приложениях: при анализе свойств сейсмических и акустических сигналов (именно здесь впервые возник термин wavelet [1]); при обработке и синтезе различных сигналов, например речевых; при анализе изображений; для изучения турбулентных полей; для сжатия больших объемов информации и т. д.

Основньми монографиями о всплесках являются [2]-[4].

На русском языке литературы по всплескам крайне мало. Отметим недавний обзор Н.М. Астафьевой [5] и предыдущую статью авторов [6].

\section{2. Обозначения и определения.}

$$
\begin{aligned}
& \|f\|_{p}=\left(\int_{\mathbb{R}^{n}}|f(x)|^{p} d x\right)^{1 / p}-\text { норма в пространстве } L^{p}\left(\mathbb{R}^{n}\right), 1 \leqslant p<\infty . \\
& \kappa_{e} \text {-характеристическая функция множества } e . \\
& |e| \text { - мера Лебега множества } e \subset \mathbb{R}^{n} \text {. } \\
& \text { Модули непрерывности: } \omega(h, f):=\sup _{|x-y|<h}|f(x)-f(y)| ;
\end{aligned}
$$
для $l \in \mathbb{N} l$-й модуль непрерьвности определяется формулой:

$$
\omega_{l}(h, f):=\sup _{|y|<h} \sup _{x}\left|\Delta_{y}^{l}(x)\right|,
$$

где $\Delta_{y}^{l}(x, f):=\sum_{j=0}^{l}(-1)^{l-j}\left(\begin{array}{l}l \\ j\end{array}\right) f(x+j y)$. 
$\langle f, g\rangle=\int_{\mathbb{R}^{n}} f(t) \overline{g(t)} d t-$ скалярное произведение.

$\mathbb{R}_{+}^{n+1}:=\left\{(x, t): x \in \mathbb{R}^{n}, t>0\right\}$ - верхнее полупространство.

$\left[x_{k}\right]_{k}$ - замыкание линейной оболочки $\left\{x_{k}\right\}_{k}$ в рассматриваемом пространстве.

ОНБ - ортонормированный базис.

$C(A)$ - пространство функций, непрерывных на $A$.

$C^{m}(A)$ - пространство функций, $m$ раз непрерьвно дифференцируемых на $A$.

$D\left(\mathbb{R}^{n}\right)$ - пространство бесконечно дифференцируемых функций с компактным носителем.

$S\left(\mathbb{R}^{n}\right)$ - пространство Шварца бесконечно дифференцируемых быстро убываюших на бесконечности функций.

Преобразование Фурье функции $f \in S\left(\mathbb{R}^{n}\right)$ определяется формулой

$$
\widehat{f}(\omega):=\int_{\mathbb{R}^{n}} e^{-i\langle x, \omega\rangle} f(x) d x, \quad \omega \in \mathbb{R}^{n},
$$

где $\langle x, \omega\rangle:=\sum_{1}^{n} x_{k} \omega_{k}$. Тогда

$$
f(x)=\frac{1}{(2 \pi)^{n}} \int_{\mathbb{R}^{n}} e^{i\langle x, \omega\rangle} \widehat{f}(\omega) d \omega, \quad x \in \mathbb{R}^{n},
$$

и

$$
(2 \pi)^{n} \int_{\mathbb{R}^{n}}|\widehat{f}(\omega)|^{2} d \omega=\int_{\mathbb{R}^{n}}|f(x)|^{2} d x \quad \text { (тождество Планшереля). }
$$

Для мультииндекса $\alpha=\left(\alpha_{1}, \ldots, \alpha_{n}\right) \partial^{\alpha}:=\left(\partial / \partial x_{1}\right)^{\alpha_{1}}+\cdots+\left(\partial / \partial x_{n}\right)^{\alpha_{n}}$ и $|\alpha|:=$ $\alpha_{1}+\cdots+\alpha_{n}$.

$$
\Delta=\frac{\partial^{2}}{\partial x_{1}^{2}}+\frac{\partial^{2}}{\partial x_{2}^{2}}+\cdots+\frac{\partial^{2}}{\partial x_{n}^{2}} .
$$

ОПРЕДЕлениЕ 2.1. Пусть $z:=x+i t$, где $(x, t) \in \mathbb{R}_{+}^{2}$. Функщия $f(z)$ принадлежит пространству Харди $\mathbb{H}^{p}\left(\mathbb{R}_{+}^{2}\right), 0<p<\infty$, если она голоморфна на $\mathbb{R}_{+}^{2}$ и

$$
\sup _{t>0}\left(\int_{\mathbb{R}}|f(x+i t)|^{p} d x\right)^{1 / p}<\infty .
$$

ОПРЕДЕлЕниЕ 2.2. Ядро Пуассона на $\mathbb{R}_{+}^{n+1}$ определяется формулой

$$
p_{a}(x):=\pi^{-(n+1) / 2} \Gamma\left(\frac{n+1}{2}\right) \frac{a}{\left(|x|^{2}+a^{2}\right)^{(n+1) / 2}}, \quad x \in \mathbb{R}^{n}, \quad a>0 .
$$

Интегралом Пуассона назьвают свертку функции $f$ с ядром $p_{a}$ :

$$
F(x, a):=\int_{\mathbb{R}^{n}} p_{a}(x-y) f(y) d y, \quad x \in \mathbb{R}^{n}, \quad a>0 .
$$

Функция $F$ является гармонической на $\mathbb{R}_{+}^{n+1}$ и совпадает на границе $\mathbb{R}_{+}^{n+1}$ с $f$.

Некасательная максимальная функция равна $F^{*}(x):=\sup _{\gamma(x)}|F(y, a)|$, где $\gamma(x):=\left\{(y, a) \in \mathbb{R}_{+}^{n}: a>|y-x|\right\}-$ конус Лузина.

Вешественные классы Харди $H^{p}\left(\mathbb{R}^{n}\right), p>0$, состоят из локально интегрируемых функций $f$, для которых $\int_{\mathbb{R}^{n}} \frac{|f(x)| d x}{1+|x|^{n+1}}<\infty$ и некасательная максимальная функция $F^{*}$ принадлежит $L^{p}\left(\mathbb{R}^{n}\right),\|f\|_{H^{p}\left(\mathbb{R}^{n}\right)}=\left\|F^{*}\right\|_{L^{p}\left(\mathbb{R}^{n}\right)}$. 
ОПРЕДЕЛЕНИЕ 2.3. Функция $f \in L_{\text {loc }}^{2}\left(\mathbb{R}^{n}\right)$ принадлежит пространству $\mathrm{BMO}\left(\mathbb{R}^{n}\right)$, если она имеет ограниченные средние осцилляции (Bounded Mean Oscillations)

$$
\sup _{B}\left(\frac{1}{|B|} \int_{B}\left|f(x)-f_{B}\right|^{2} d x\right)^{1 / 2}=\|f\|_{\mathrm{BMO}}<\infty
$$

где $\sup$ берется по всем шарам $B \subset \mathbb{R}^{n}$ и $f_{B}:=\frac{1}{|B|} \int_{B} f(x) d x$.

ОПРЕДЕЛЕНИЕ 2.4. Преобразованием Гильберта $H u$ действительной функщии $u$ называют действительную функцию $v$ такую, что функция $u(x)+i v(x)$ является следом на $\mathbb{R}$ голоморфной функции $f(x+i y)$. В терминах преобразования Фурье это эквивалентно тому, что $\widehat{v}(\xi)=-i \operatorname{sign}(\xi) \widehat{u}(\xi)$.

ОПРЕДЕЛЕНИЕ 2.5. Линейньй оператор, определенньй и ограниченньй в $L^{2}\left(\mathbb{R}^{n}\right)$, называется оператором Кальдерона-Зигмунда, если сушествует функция $K(x, y)$, определенная при $x, y \in \mathbb{R}^{n}, x \neq y$, такая, что для некоторого $\alpha>0$

$$
\begin{gathered}
|K(x, y)| \leqslant \frac{C}{|x-y|^{n}} ; \\
\left|K(x, y)-K\left(x, y^{\prime}\right)\right| \leqslant C \frac{\left|y-y^{\prime}\right|^{\alpha}}{|x-y|^{n+\alpha}}, \quad\left|y-y^{\prime}\right| \leqslant \frac{1}{2}|x-y| \\
\left|K(x, y)-K\left(x^{\prime}, y\right)\right| \leqslant C \frac{\left|x-x^{\prime}\right|^{\alpha}}{|x-y|^{n+\alpha}}, \quad\left|x-x^{\prime}\right| \leqslant \frac{1}{2}|x-y|
\end{gathered}
$$

и для любой функции $f \in D\left(\mathbb{R}^{n}\right)$ и для любого $x \notin \operatorname{supp} f T f(x)=\int_{\mathbb{R}^{n}} K(x, y) f(y) d y$.

ТеОрема 2.1. Пусть $T$ - оператор Кальдерона-Зигмунда. Тогда при $1<p<\infty$ $\|T f\|_{p} \leqslant C_{p}\|T\|\|f\|_{p}$, zдe $\|T\|:=\|T\|_{2}+\inf C$.

ОПРЕДЕЛЕНИЕ 2.6. Если $s=0$, то пространство Соболева $W_{2}^{s}\left(\mathbb{R}^{n}\right)$ совпадает с $L_{2}\left(\mathbb{R}^{n}\right)$. Для $s \in \mathbb{N}$ функция $f$ принадлежит $W_{2}^{s}\left(\mathbb{R}^{n}\right)$, если она принадлежит $L_{2}\left(\mathbb{R}^{n}\right)$ вместе со всеми своими производными $\partial^{\alpha} f$ (в смысле обобщенных функций) с $|\alpha| \leqslant s$.

Известно, что последнее эквивалентно $\int_{\mathbb{R}^{n}}\left(1+|\omega|^{2}\right)^{s}|\widehat{f}(\omega)|^{2} d \omega<\infty$, что принимается в качестве определения при произвольном $s \geqslant 0$.

Пространств $W_{2}^{s}\left(\mathbb{R}^{n}\right)$ для $s<0$ определяется как двойственное к $W_{2}^{-s}\left(\mathbb{R}^{n}\right)$. Применяя тождество Парсеваля, легко получить, что умеренное распределение $S$ принадлежит $W_{2}^{s}\left(\mathbb{R}^{n}\right)$ тогда и только тогда, когда его преобразование Фурье $\widehat{S}$ принадлежит $L_{\mathrm{loc}}^{2}\left(\mathbb{R}^{n}\right)$ и удовлетворяет условию $\int_{\mathbb{R}^{n}}|\widehat{S}(\omega)|^{2}\left(1+|\omega|^{2}\right)^{s} d \omega<\infty$.

ОПРЕДЕЛЕНИЕ 2.7. Пусть $1 \leqslant p \leqslant \infty$. Если $s \geqslant 0$ и $s$ - целое, то пространство $W_{p}^{s}$ состоит из функций $f$ таких, что $f$ и все ее производные $\partial^{\alpha} f$ с $|\alpha| \leqslant s$ принадлежат $L^{p}\left(\mathbb{R}^{n}\right)$. Это эквивалентно условию $(I-\Delta)^{s / 2} f \in L^{p}\left(\mathbb{R}^{n}\right)$. Последнее условие определяет $W_{p}^{s}$ для произвольного действительного $s$. 
ОПРЕДЕЛЕНИЕ 2.8. Пусть $1 \leqslant p, q \leqslant \infty, s>0$ и $s<m \in \mathbb{N}$. Функщия $f$ принадлежит пространству Бесова $B_{p}^{s, q}$, если $f \in L^{p}\left(\mathbb{R}^{n}\right)$ и сушествуют последовательность положительных чисел $\varepsilon_{j}$ из $l^{q}(\mathbb{N})$ и последовательность функций $f_{j}$ из $L^{p}\left(\mathbb{R}^{n}\right)$ такие, что

$$
\left\|f-f_{j}\right\|_{p} \leqslant \varepsilon_{j} 2^{-j s}, \quad j \in \mathbb{N},
$$

и

$$
\left\|\partial^{\alpha} f_{j}\right\|_{p} \leqslant \varepsilon_{j} 2^{(m-s) j}
$$

для любого мультииндекса $\alpha \in \mathbb{N}^{n} \mathrm{c}|\alpha|=m$.

ОПРеДЕЛЕниЕ 2.9. При $0<s<1$ пространство Гёльдера $\mathbb{C}^{s}\left(\mathbb{R}^{n}\right)$ состоит из функций $f \in C\left(\mathbb{R}^{n}\right)$ таких, что $\sup _{x \in \mathbb{R}^{n}}|f(x)|<\infty$ и $\sup _{h>0} \omega(h) / h^{s}<\infty$.

$\mathbb{C}^{1}\left(\mathbb{R}^{n}\right)$ - это класс Зигмунда:

$$
\mathbb{C}^{1}\left(\mathbb{R}^{n}\right):=\left\{f \in C\left(\mathbb{R}^{n}\right): \sup _{x \in \mathbb{R}^{n}}|f(x)|<\infty, \sup _{h>0} \frac{\omega_{2}(h, f)}{h}<\infty\right\} .
$$

Для $s \in(m, m+1], m \in \mathbb{N}$,

$$
\mathbb{C}^{s}\left(\mathbb{R}^{n}\right):=\left\{f \in C^{m}\left(\mathbb{R}^{n}\right): \forall|\alpha| \leqslant m \partial^{\alpha} f \in \mathbb{C}^{s-m}\right\} .
$$

3. Прототипы всплесков в работах Лузина и Кальдерона. Одно из первых появлений всплесков в математическом анализе относится к тридцатым годам. Хорошо известная характеризация пространств $\mathbb{H}^{p}$ в терминах интеграла площадей или функции Лузина [7] содержит прототипы всплесков (см. [8]).

Работа Н.Н. Лузина [7] посвящена анализу и синтезу функций из $\mathbb{H}^{p}\left(\mathbb{R}_{+}^{2}\right)$ при помощи интегрального представления

$$
f(x)=\frac{2 i}{\pi} \iint_{\mathbb{R}_{+}^{2}} t f^{\prime}(y+i t) \frac{d y d t}{(x-y+i t)^{2}}, \quad x \in \mathbb{R} .
$$

Представлению (3.1) можно придать всплесковый вид (1.1). Рассмотрим функцию $\psi(t):=(t+i)^{-2}, t \in \mathbb{R}$, которая осциллирует $\left(\right.$ т.е. $\left.\int_{\mathbb{R}} \psi(t) d t=0\right)$, регулярна и локализована. Благодаря этим свойствам родоначальники всплесков А. Гроссман (A. Grossman) и Ж. Морле (J. Morlet) [1] назвали $\psi$ - wavelet, буквально маленькая (имеется в виду продолжительность) волна.

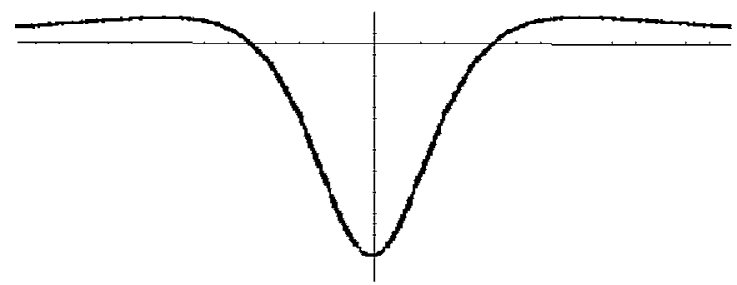

Рис. 1. График $\operatorname{Re}(\psi(t))$ 


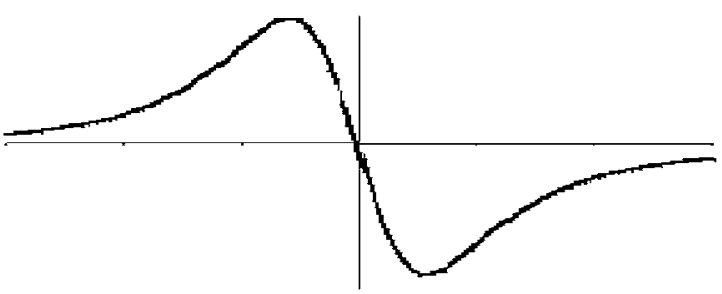

Рис. 2. График $\operatorname{Im}(\psi(t))$

Рассмотрим $\psi_{a, b}(t):=a^{-1 / 2} \psi\left(\frac{t-b}{a}\right)=a^{3 / 2}(t-b+i a)^{-2}$ для $a>0, b \in \mathbb{R}$. Определим

$$
\left(W_{\psi} f\right)(a, b):=\left\langle f, \psi_{a, b}\right\rangle=2 \pi i a^{3 / 2} f^{\prime}(b+i a) .
$$

В силу (3.1) функция $f$ представима в виде суперпозиции всплесков $\psi_{a, b}(t)$ с коэффициентами $\left(W_{\psi} f\right)(a, b)$. Для любой функции $f \in \mathbb{H}^{2}\left(\mathbb{R}_{+}^{2}\right)$ имеем

$$
\begin{aligned}
f(x) & =\frac{2 i}{\pi} \iint_{\mathbb{R}_{+}^{2}} a f^{\prime}(b+i a) \frac{d b d a}{(x-b+i a)^{2}} \\
& =\frac{1}{\pi^{2}} \int_{0}^{\infty}\left[\int_{\mathbb{R}}\left(W_{\psi} f\right)(a, b) \psi_{a, b}(x) d b\right] \frac{d a}{a^{2}} .
\end{aligned}
$$

Представление (3.1) позволяет охарактеризовать функции из $\mathbb{H}^{p}\left(\mathbb{R}_{+}^{2}\right)$.

ОПРЕДЕЛЕнИЕ 3.1. Интегралом площадей или функцией Лузина называется

$$
(S f)(x)=\left(\iint_{\gamma(x)}\left|f^{\prime}(y+i t)\right|^{2} d y d t\right)^{1 / 2}, \quad x \in \mathbb{R}
$$

где $\gamma(x)=\left\{(y, t) \in \mathbb{R}^{2}: t>|y-x|\right\}-$ конус Лузина.

Teорема 3.1. Пусть $0<p<\infty$. Тогда $f \in \mathbb{H}^{p}\left(\mathbb{R}_{+}^{2}\right)$, если и только если $S f \in L^{p}(\mathbb{R})$.

Для $1<p<\infty$ это классический результат (см. [9], [10]). Для $0<p \leqslant 1$ это результат А. Кальдерона [11].

Рассмотренные формулы обобщаются в тождестве Кальдерона [12].

Пусть $\psi \in L^{1}\left(\mathbb{R}^{n}\right), \int_{\mathbb{R}^{n}} \psi(x) d x=0$ и преобразование Фурье $\widehat{\psi}(\omega), \omega \in \mathbb{R}^{n}$, удовлетворяет условию

$$
\int_{0}^{\infty}|\widehat{\psi}(t \omega)|^{2} \frac{d t}{t}=1 \text { для любых } \omega \neq 0 .
$$

Например, если $\psi$ достаточно регулярна, локализована, имеет нулевое среднее и радиальна, то существует константа $c>0$ такая, что $c \psi(x)$ удовлетворяет (3.5).

Положим $\psi_{a}(x)=a^{-n / 2} \psi\left(\frac{x}{a}\right)$ и $\psi_{a, b}(x)=a^{-n / 2} \psi\left(\frac{x-b}{a}\right)$. Следуя [1], определим

$$
\left(W_{\psi} f\right)(a, b):=\left\langle f, \psi_{a, b}\right\rangle .
$$


Тогда имеет место следующая формула восстановления:

$$
f(x)=\int_{0}^{\infty}\left[\int_{\mathbb{R}^{n}}\left(W_{\psi} f\right)(a, b) \psi_{a, b}(x) d b\right] \frac{d a}{a^{n+1}} .
$$

Эквивалентная формулировка для (3.7) имеет вид

$$
I=\int_{0}^{\infty} Q_{a} Q_{a}^{*} \frac{d a}{a^{n+1}},
$$

где $I$ - тождественный оператор, $Q_{a}(f)=f * \psi_{a}$ - оператор свертки, а $Q_{a}^{*}$ - сопряженный к $Q_{a}$ оператор. Тождество Кальдерона (3.8) имеет важные приложения в анализе классических функциональных пространств посредством условий, используюших модуль градиента гармонического продолжения (см. [9]). Например, для полугрупшы Пуассона $P_{a} f(x):=F(x, a)$ (определение 2.2 ) оператор $Q_{a}$ имеет вид $Q_{a}=$ $-a(\partial / \partial a) P_{a}$.

В формулах (3.6) и (3.7) функщии $\psi_{a, b}, b \in \mathbb{R}^{n}, a>0$, используются так, как будто они образуют ортонормированньй базис в $L^{2}\left(\mathbb{R}^{n}\right)$ : коэффищиенты разложения вычисляются по формуле (3.6), а представление функции $f$ в терминах этих коэффициентов дается (3.7).

Можно заменить избыточное множество функций $\psi_{a, b}, b \in \mathbb{R}^{n}, a>0$, на ортогональньй базис $\psi_{\lambda}, \lambda \in \Lambda$, который конструируется по тем же алгебраическим правилам (путем сжатий и сдвигов). Ортонормированньй базис $\psi_{\lambda}, \lambda \in \Lambda$, оказывается универсальным безусловным базисом для классических пространств функций и распределений.

4. Преобразование Габора. Другим предшественником всплесковых преобразований является преобразование Габора. Пусть функция $f$ принадлежит $L^{2}(\mathbb{R})$ и $\widehat{f}$ - ее преобразование Фурье (2.1). Для вычисления $\widehat{f}(\omega)$ в любой точке $\omega$ в равной степени используются все значения $f$, поэтому $\widehat{f}$ не отражает изменений частотных характеристик $f$ по времени $t$. Кроме того, изменение функции $f$ в сколь угодно малой окрестности произвольной точки $t_{0}$ приводит к изменению всего спектра.

Для исправления этих недостатков Д. Габор рассмотрел в [13] следующее преобразование. Пусть

$$
g_{\alpha}(t):=\frac{1}{2 \sqrt{\pi \alpha}} e^{-\frac{t^{2}}{4 \alpha}},
$$

где $\alpha$ - фиксированный параметр.

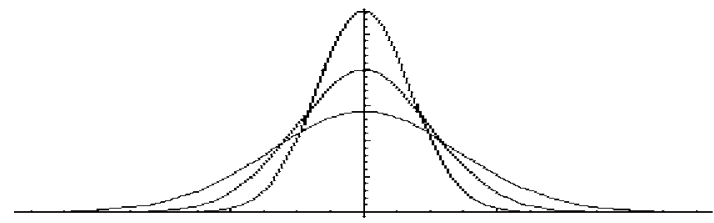

Рис. 3. График $g_{\alpha}, \alpha=1, \frac{1}{2}, \frac{1}{4}$ 
Функция $g_{\alpha}$ используется в качестве так называемого временного окна. Преобразование Габора функции $f \in L^{2}(\mathbb{R})$ определяется формулой

$$
\left(\Gamma_{b}^{\alpha} f\right)(\omega):=\int_{\mathbb{R}}\left(e^{-i \omega t} f(t)\right) g_{\alpha}(t-b) d t
$$

Ясно, что $\left(\Gamma_{b}^{\alpha}\right)(\omega)$ локализует преобразование Фурье вокруг точки $t=b$.

Так как $\int_{\mathbb{R}} g_{\alpha}(t-b) d b=\int_{\mathbb{R}} g_{\alpha}(x) d x=1$, то $\int_{\mathbb{R}}\left(\Gamma_{b}^{\alpha} f\right)(\omega) d b=\widehat{f}(\omega), \omega \in \mathbb{R}$. Таким образом, преобразование Габора разлагает преобразование Фурье на локальную спектральную информацию.

Приведем общее определение оконной функции. Функцию $w$ назьвают оконной, если $w \in L^{2}(\mathbb{R})$ и $x w(x) \in L^{2}(\mathbb{R})$. Для количественной характеристики локализованности функции $w$ используются следующие величины:

$$
\begin{aligned}
t^{*} & :=\frac{1}{\|w\|_{2}^{2}} \int_{\mathbb{R}} x|w(x)|^{2} d x-\text { центр; } \\
\Delta_{w} & :=\frac{1}{\|w\|_{2}^{2}}\left\{\int_{\mathbb{R}}\left(x-t^{*}\right)^{2}|w(x)|^{2} d x\right\}^{1 / 2}-\text { радиус. }
\end{aligned}
$$

Ширина функщии $w$ полагается равной двум радиусам. Легко вычислить, что $\Delta g_{\alpha}=\sqrt{\alpha}$.

Преобразование Габора можно интерпретировать следующим образом. Пусть $G_{b, \omega}^{\alpha}(t):=e^{i \omega t} g_{\alpha}(t-b)$.

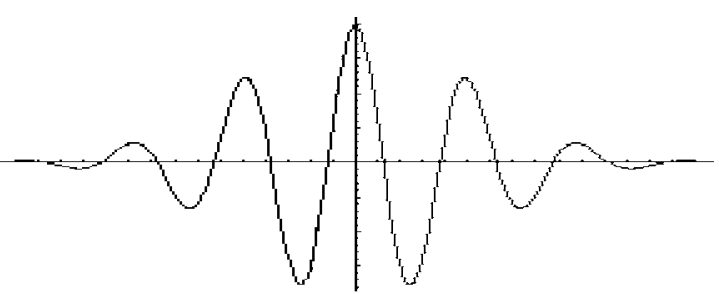

Рис. 4. График $\operatorname{Re}\left(G_{0,2 \pi}^{0.5}(t)\right)$

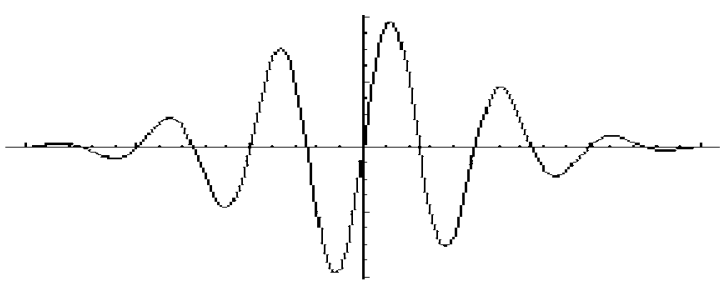

Рис. 5. График $\operatorname{Im}\left(G_{0,2 \pi}^{0.5}(t)\right)$ 
Тогда

$$
\left(\Gamma_{b}^{\alpha} f\right)(\omega)=\int_{\mathbb{R}} f(t) \overline{G_{b, \omega}^{\alpha}(t)} d t
$$

Преимушество (4.3) состоит в возможности применить тождество Планшереля. Действительно, $\widehat{G_{b, \omega}^{\alpha}}(\eta)=e^{-i b(\eta-\omega)} e^{-\alpha(\eta-\omega)^{2}}$. Поэтому

$$
\begin{aligned}
\left(\Gamma_{b}^{\alpha} f\right)(\omega) & =\frac{1}{2 \pi} \int_{\mathbb{R}} \widehat{f}(\eta) e^{i b(\eta-\omega)} e^{-\alpha(\eta-\omega)^{2}} d \eta \\
& =\frac{e^{-i b \omega}}{2 \sqrt{\pi \alpha}} \int_{\mathbb{R}}\left(e^{i b \eta} \widehat{f}(\eta)\right) g_{1 /(4 \alpha)}(\eta-\omega) d \eta=\frac{e^{-i b \omega}}{2 \sqrt{\pi \alpha}}\left(\Gamma_{\omega}^{1 /(4 \alpha)} \widehat{f}\right)(-b) .
\end{aligned}
$$

Таким образом, преобразование Габора функции $f$ с оконной функцией $g_{\alpha}$ в точке $t=b$ с точностью до множителя совпадает с преобразованием Габора функции $\widehat{f}$ с оконной функцией $g_{1 /(4 \alpha)}$ в точке $\eta=\omega$. Произведение ширины окна $g_{\alpha}$ на ширину окна $g_{1 /(4 \alpha)}$ равно 2. Декартово произведение $[b-\sqrt{\alpha}, b+\sqrt{\alpha}] \times\left[\omega-\frac{1}{2 \sqrt{\alpha}}, \omega+\frac{1}{2 \sqrt{\alpha}}\right]$ этих двух окон называют прямоугольным время-частотным окном. Ширина $2 \sqrt{\alpha}$ временного окна называется шириной время-частотного окна, а ширина $1 / \sqrt{\alpha}$ частотного окна называется высотой время-частотного окна. Отметим, что ширина время-частотного окна в преобразовании Габора не изменяется при наблюдении спектра на всех частотах.

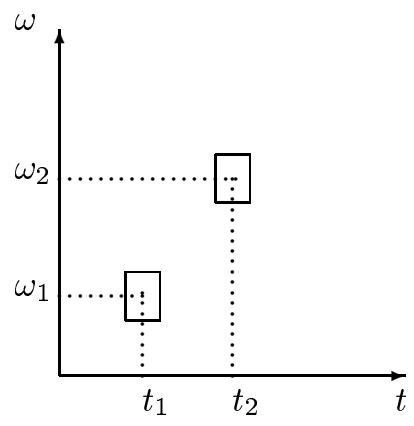

Рис. 6. Окна Габора

5. Оконное преобразование Фурье. Преобразование Габора можно обобшить. Пусть

$$
w \in L^{2}(\mathbb{R}) \text { и } t w(t) \in L^{2}(\mathbb{R}) .
$$

Оконным преобразованием Фурье называется

$$
\left(\widetilde{\Gamma_{b}}\right)(\omega):=\int_{\mathbb{R}}\left(e^{-i \omega t} f(t)\right) \overline{w(t-b)} d t .
$$

Полагая

$$
\begin{aligned}
W_{b, \omega}(t) & :=e^{i \omega t} w(t-b), \\
V_{b, \omega}(\eta) & :=\frac{1}{2 \pi} \widehat{W}_{b, \omega}(\eta)=\frac{e^{i b \omega}}{2 \pi} e^{-i b \eta} \widehat{w}(\eta-\omega),
\end{aligned}
$$


имеем

$$
\left(\widetilde{\Gamma_{b}}\right)(\omega):=\int_{\mathbb{R}} f(t) \overline{W_{b, \omega}(t)} d t=\int_{\mathbb{R}} \widehat{f}(\eta) \overline{V_{b, \omega}(\eta)} d \eta
$$

Таким образом, оконное преобразование дает локальную информацию о $f$ во временном окне $\left[t^{*}+b-\Delta_{w}, t^{*}+b+\Delta_{w}\right]$ и локальную информацию о $\widehat{f}$ в частотном окне $\left[\omega^{*}+\omega-\Delta_{\widehat{w}}, \omega^{*}+\omega+\Delta_{\widehat{w}}\right]$, где $\omega^{*}-$ центр $\widehat{w}$. Если и $w$, и $\widehat{w}$ удовлетворяют $(5.1)$, то время-частотное окно $\left[t^{*}+b-\Delta_{w}, t^{*}+b+\Delta_{w}\right] \times\left[\omega^{*}+\omega-\Delta_{\widehat{w}}, \omega^{*}+\omega+\Delta_{\widehat{w}}\right]$ имеет постоянную ширину $2 \Delta_{w}$ и постоянную плошадь $4 \Delta_{w} \Delta_{\widehat{w}}$.

Произведение $\Delta_{w} \Delta_{\widehat{w}}$ характеризует время-частотную локализацию $w$ и назьвается константой неопределенности $w$. Напомним принцип неопределенности (см., например, [14]).

Теорема 5.1. Пусть $w \in L^{2}(\mathbb{R})$ удовлетворяет (5.1) вместе $c \widehat{w}$. Тогда $\Delta_{w} \Delta_{\widehat{w}} \geqslant \frac{1}{2}$. Более того, равенство достигается тогда и только тогда, когда $w(t)=c e^{i a t} g_{\alpha}(t-b)$, где $c \neq 0, \alpha>0 ; a, b \in \mathbb{R}$.

Таким образом, преобразование Габора имеет наименьшее время-частотное окно. В некоторых приложениях приходится использовать большие окна для получения дополнительных свойств, например, легкости вычислений.

Приведем формулу обрашения для оконных преобразований Фурье.

ТеОРема 5.2. Пусть $w \in L^{2}(\mathbb{R}),\|w\|_{2}=1, w$ и $\widehat{w}$ удовлетворяют (5.1). Тогда

$$
\int_{\mathbb{R}} \int_{\mathbb{R}}\left\langle f, W_{b, \omega}\right\rangle \overline{\left\langle g, W_{b, \omega}\right\rangle} d b d \omega=2 \pi\langle f, g\rangle
$$

Если $x$ - точка непрерьвности $f$, то

$$
f(x)=\frac{1}{2 \pi} \int_{\mathbb{R}} \int_{\mathbb{R}}\left[e^{i \omega x}\left(\widetilde{\Gamma_{b}} f\right)(\omega)\right] w(x-b) d \omega d b .
$$

Как уже отмечалось выше, в оконном преобразовании Фурье ширина окна не изменяется при изучении любой частотной полосы. Однако, частота прямо пропорциональна числу периодов в единищу времени, поэтому для локализации высокочастотных изменений естественно брать более узкое окно для увеличения точности выгислений, а для низкочастотных - более широкое для получения полной информации. Таким образом, оконное преобразование Фурье не применимо к изучению сигналов, содержащих как очень высокие, так и очень низкие частоты.

Данньй недостаток исправляется в интегральном всплесковом преобразовании, время-частотное окно которого автоматически сужается при наблюдении высокочастотных изменений и расширяется для изучения низкочастотных.

\section{6. Интегральное всплесковое преобразование.}

ОПРЕДЕЛЕНИЕ 6.1. Функцию $\psi \in L^{2}(\mathbb{R})$ назьвают базовым всплеском, если она удовлетворяет условию:

$$
C_{\psi}:=\int_{\mathbb{R}} \frac{|\widehat{\psi}(\omega)|^{2}}{|\omega|} d \omega<\infty
$$


При помоши базового всплеска определяется интегральное всплесковое преобразование $\left(\right.$ ИВП) на $L^{2}(\mathbb{R})$ :

$$
\left(W_{\psi} f\right)(b, a):=|a|^{-1 / 2} \int_{\mathbb{R}} f(t) \overline{\psi\left(\frac{t-b}{a}\right)} d t, \quad f \in L^{2}(\mathbb{R}),
$$

где $a, b \in \mathbb{R}$ с $a \neq 0$.

Полагая

$$
\psi_{b, a}(t):=|a|^{-1 / 2} \psi\left(\frac{t-b}{a}\right)
$$

имеем

$$
\left(W_{\psi} f\right)(b, a)=\left\langle f, \psi_{b, a}\right\rangle
$$

В дальнейшем будем считать, что и $\psi$, и $\widehat{\psi}$ удовлетворяют (5.1). Тогда если центр и радиус $\psi$ равны $t^{*}$ и $\Delta_{\psi}$ соответственно, то функция $\psi_{b, a}$ является оконной с центром в $b+a t^{*}$ и радиусом $a \Delta_{\psi}$. Значит, ИВП дает локальную информацию о функщии $f$ с временным окном $\left[b+a t^{*}-a \Delta_{\psi}, b+a t^{*}+a \Delta_{\psi}\right]$. Это окно сужается при малых значениях $a$ и расширяется при больших.

Рассмотрим теперь

$$
\frac{1}{2 \pi} \widehat{\psi}_{b, a}(\omega)=\frac{|a|^{-1 / 2}}{2 \pi} \int_{\mathbb{R}} e^{-i \omega t} \psi\left(\frac{t-b}{a}\right) d t=\frac{a|a|^{-1 / 2}}{2 \pi} e^{-i b \omega} \widehat{\psi}(a \omega)
$$

и предположим, что центр и радиус функции $\widehat{\psi}$ равны $\omega^{*}$ и $\Delta_{\widehat{\psi}}$ соответственно. Полагая $\eta(\omega):=\widehat{\psi}\left(\omega+\omega^{*}\right)$, получаем оконную функцию $\eta$ с центром в нуле и радиусом $\Delta_{\widehat{\psi}}$. Применяя тождество Планшереля к (6.2), имеем

$$
\left(W_{\psi} f\right)(b, a)=\frac{a|a|^{-1 / 2}}{2 \pi} \int_{\mathbb{R}} \widehat{f}(\omega) e^{i b \omega} \overline{\eta\left(a\left(\omega-\frac{\omega^{*}}{a}\right)\right)} d \omega
$$

Ясно, что оконная функция $\eta\left(a\left(\omega-\frac{\omega^{*}}{a}\right)\right)=\eta\left(a \omega-\omega^{*}\right)=\widehat{\psi}(a \omega)$ имеет радиус $\frac{1}{a} \Delta_{\widehat{\psi}}$. Поэтому, с точностью до множителя $\frac{a|a|^{-1 / 2}}{2 \pi}$ и линейного фазового сдвига $e^{i b \omega}$, ИВП $W_{\psi} f$ дает локальную информацию о $\widehat{f}$ с частотным окном

$$
\left[\frac{\omega^{*}}{a}-\frac{1}{a} \Delta_{\widehat{\psi}}, \frac{\omega^{*}}{a}+\frac{1}{a} \Delta_{\widehat{\psi}}\right]
$$

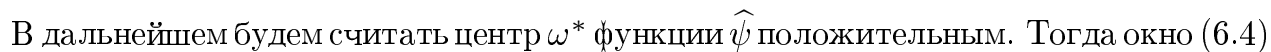
является частотной полосой (или октавой) с центральной частотой $\omega^{*} / a$ и шириной полосы $2 \Delta_{\widehat{\psi}} / a$. Важно, что отношение

$$
\frac{\text { центральная частота }}{\text { ширина }}=\frac{\omega^{*} / a}{2 \Delta_{\widehat{\psi}} / a}=\frac{\omega^{*}}{2 \Delta_{\widehat{\psi}}}
$$




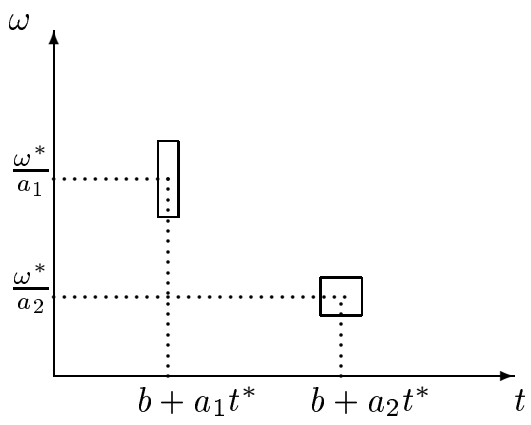

Рис. 7. Окна ИВП

не зависит от масштаба $a$.

Итак, для ИВП имеем прямоугольное время-частотное окно

$$
\left[b+a t^{*}-a \Delta_{\psi}, b+a t^{*}+a \Delta_{\psi}\right] \times\left[\frac{\omega^{*}}{a}-\frac{1}{a} \Delta_{\widehat{\psi}}, \frac{\omega^{*}}{a}+\frac{1}{a} \Delta_{\widehat{\psi}}\right] .
$$

Отметим еще раз, что окно сужается для выявления высокочастотных явлений и расширяется для исследования низкочастотных.

Следуюший результат показывает, как восстановить функцию по ее ИВП.

Tеорема 6.1. Пусть $\psi-$ базовый всплеск. Тогда для любых $f, g \in L^{2}(\mathbb{R})$

$$
\int_{\mathbb{R}} \int_{\mathbb{R}}\left[\left(W_{\psi} f\right)(b, a) \overline{\left(W_{\psi} g\right)(b, a)}\right] \frac{d a}{a^{2}} d b=C_{\psi}\langle f, g\rangle .
$$

Более того, для любой $f \in L^{2}(\mathbb{R})$ и любой точки $x$, в которой $f$ непрерывна,

$$
f(x)=\frac{1}{C_{\psi}} \int_{\mathbb{R}} \int_{\mathbb{R}}\left[\left(W_{\psi} f\right)(b, a)\right] \psi_{b, a}(x) \frac{d a}{a^{2}} d b,
$$

где $\psi_{b, a}$ определены в (6.1).

ДоКАЗАТЕльСТво. В силу (6.3) и тождества Планшереля

$$
\left(W_{\psi} f\right)(b, a)=\left\langle f, \psi_{b, a}\right\rangle=\frac{1}{2 \pi} \int_{\mathbb{R}} \widehat{f}(\omega) a|a|^{-1 / 2} e^{-i b \omega} \widehat{\psi}(a \omega) d \omega=\frac{1}{2 \pi} \widehat{F}_{a}(b),
$$

где $F_{a}(\omega):=a|a|^{-1 / 2} \widehat{f}(\omega) \widehat{\psi}(a \omega)$. Поэтому, опять используя тождество Планшереля, имеем

$$
\begin{aligned}
\int_{\mathbb{R}} \int_{\mathbb{R}}\left[\left(W_{\psi} f\right)(b, a) \overline{\left(W_{\psi} g\right)(b, a)}\right] \frac{d a}{a^{2}} d b & =\frac{1}{4 \pi^{2}} \int_{\mathbb{R}} \int_{\mathbb{R}} \widehat{F}_{a}(b) \overline{\widehat{G}_{a}(b)} d b \frac{d a}{a^{2}} \\
& =\frac{1}{2 \pi} \int_{\mathbb{R}}\left\{\widehat{f}(\omega) \overline{\widehat{g}(\omega)} \int_{\mathbb{R}} \frac{|\widehat{\psi}(a \omega)|^{2}}{|a|} d a\right\} d \omega \\
& =\frac{1}{2 \pi} C_{\psi}\langle\widehat{f}, \widehat{g}\rangle=C_{\psi}\langle f, g\rangle .
\end{aligned}
$$

Второе утверждение теоремы следует из первого, если взять в качестве функций $g$ гауссовские функции $g_{\alpha}(\cdot-x)($ см. (4.1)) и устремить $\alpha$ к нулю. 
ЗАмЕчАниЕ 6.1 . Во всплесковых преобразованиях основную роль играет коммутативная локально компактная группа отображений $\mathbb{R} \mapsto \mathbb{R}$ вида $g(x)=a x+b$, $a \in \mathbb{R} \backslash\{0\}, b \in \mathbb{R}, x \in \mathbb{R}$, с мерой Хаара $a^{-2} d a d b$. Т. П. Лукашенко [15] обобшил теорему 6.1 на произвольную коммутативную локально компактную групу.

При анализе физических сигналов рассматриваются только положительные частоты. Так как частота обратно пропоршиональна параметру сжатия $a: \omega=\omega^{*} / a$, то в этом случае необходимо рассматривать только положительные $a$ и восстанавливать сигнал по значениям $\left(W_{\psi} f\right)(b, a), a>0$. Для этого на базовьй всплеск надо наложить дополнительное требование:

$$
\int_{0}^{\infty} \frac{|\widehat{\psi}(\omega)|^{2}}{\omega} d \omega=\int_{0}^{\infty} \frac{|\widehat{\psi}(w)|^{2}}{\omega} d \omega=\frac{1}{2} C_{\psi}<\infty
$$

ТеОРема 6.2. Пусть базовый всплеск $\psi$ удовлетворяет (6.5). Тогда для любых $f, g \in L^{2}(\mathbb{R})$

$$
\int_{0}^{\infty}\left[\int_{-\infty}^{\infty}\left(W_{\psi} f\right)(b, a) \overline{\left(W_{\psi} g\right)(b, a)} d b\right] \frac{d a}{a^{2}}=\frac{1}{2} C_{\psi}\langle f, g\rangle .
$$

Более того, для любой $f \in L^{2}(\mathbb{R})$ и любой точки $x$, в которой $f$ непрерывна,

$$
f(x)=\frac{2}{C_{\psi}} \int_{0}^{\infty}\left[\int_{\mathbb{R}}\left(W_{\psi} f\right)(b, a) \psi_{b, a}(x) d b\right] \frac{d a}{a^{2}},
$$

где $\psi_{b, \text { a }}$ определены в $(6.1)$.

7. Двоичные всплески и формулы обращения. При анализе сигналов частотную ось часто разбивают на дизъюнктные частотные полосы (или октавы). Рассмотрим двоичное разбиение: $(0, \infty)=\bigcup_{j=-\infty}^{\infty}\left(2^{j} \Delta_{\widehat{\psi}}, 2^{j+1} \Delta_{\widehat{\psi}}\right]$, где $\Delta_{\widehat{\psi}}>0$ - радиус преобразования Фурье $\widehat{\psi}$ базового всплеска $\psi$. Мы, как обычно, предполагаем, что $\widehat{\psi}$ удовлетворяет (5.1). Заметим, что, не ограничивая обшности, можно считать $\omega^{*}=$ $3 \Delta_{\widehat{\psi}}$ (достаточно применить к $\psi$ соответствуюший фазовый сдвиг: $\psi^{0}(t):=e^{i \alpha t} \psi(t)$ ). Тогда при $a_{j}:=2^{-j}$ имеем

$$
\left(\frac{\omega^{*}}{a_{j}}-\frac{1}{a_{j}} \Delta_{\widehat{\psi}}, \frac{\omega^{*}}{a_{j}}+\frac{1}{a_{j}} \Delta_{\widehat{\psi}}\right]=\left(2^{j+1} \Delta_{\widehat{\psi}}, 2^{j+2} \Delta_{\widehat{\psi}}\right] .
$$

Центральная частота этой полосы равна $\omega_{j}:=\frac{\omega^{*}}{a_{j}}=3 \times 2^{j} \Delta_{\widehat{\psi}}$.

При дополнительных предположениях на базовьй всплеск $\psi$ оказьвается возможным восстановить функцию, используя значения ИВП $\left(W_{\psi} f\right)(b, a)$ только на дискретном множестве частот $\left\{\omega_{j}=3 \times 2^{j} \Delta_{\widehat{\psi}}\right\}_{j \in \mathbb{Z}}$ (т.е. $a=a_{j}, j \in \mathbb{Z}$ ). 
ОПРЕДЕлЕнИЕ 7.1. Функция $\psi \in L^{2}(\mathbb{R})$ назьвается двоичным всплеском, если сушествуют две положительные константы $A$ и $B, 0<A \leqslant B<\infty$, такие, что почти всюду (п.в.)

$$
A \leqslant \sum_{j \in \mathbb{Z}}\left|\widehat{\psi}\left(2^{-j} \omega\right)\right|^{2} \leqslant B
$$

Условие (7.1) назьвают условием устойчивости.

Пусть $f^{-}(x):=f(-x)$. Определим нормированное ИВП

$$
\left(W_{j}^{\psi} f\right)(b):=2^{j / 2}\left(W_{\psi} f\right)\left(b, 2^{-j}\right)=2^{j}\left(f * \overline{\psi^{-}\left(2^{j} \cdot\right)}\right)(b) .
$$

Используя тождество Планшереля, легко показать, что (7.1) эквивалентно

$$
A\|f\|_{2}^{2} \leqslant \sum_{j \in \mathbb{Z}}\left\|W_{j}^{\psi} f\right\|_{2}^{2} \leqslant B\|f\|_{2}^{2}, \quad f \in L^{2}(\mathbb{R}) .
$$

Следующий результат показьвает, что двоичньй всплеск всегда является базоBыM.

ТЕОРема 7.1. Пусть $\psi$ удовлетворяет (7.1). Тогда $\psi$ является базовым всплеском и

$$
A \ln 2 \leqslant \int_{0}^{\infty} \frac{|\widehat{\psi}(\omega)|^{2}}{\omega} d \omega, \quad \int_{0}^{\infty} \frac{|\widehat{\psi}(-\omega)|^{2}}{\omega} \leqslant B \ln 2 .
$$

Более того, если $A=B$, mo

$$
C_{\psi}:=\int_{\mathbb{R}} \frac{|\widehat{\psi}(\omega)|^{2}}{|\omega|} d \omega=2 A \ln 2 .
$$

Для восстановления $f \in L^{2}(\mathbb{R})$ по значениям ИВП $\left(W_{\psi} f\right)\left(b, 2^{-j}\right), j \in \mathbb{Z}$, легче всего использовать другой двоичный всплеск $\psi^{*}$, определяемьй в образах Фурье следуюшим образом:

$$
\widehat{\psi}^{*}(\omega):=\frac{\widehat{\psi}(\omega)}{\sum_{k \in \mathbb{Z}}\left|\widehat{\psi}\left(2^{-k} \omega\right)\right|^{2}} .
$$

Тогда

$$
\begin{aligned}
& \sum_{j \in \mathbb{Z}} \int_{\mathbb{R}}\left(W_{j}^{\psi} f\right)(b)\left\{2^{j} \psi^{*}\left(2^{j}(x-b)\right)\right\} d b \\
& \left.=\sum_{j \in \mathbb{Z}} \frac{1}{2 \pi} \int_{\mathbb{R}} \widehat{\left(W_{j}^{\psi} f\right.}\right)(\omega) \widehat{\psi}^{*}\left(2^{-j} \omega\right) e^{i x \omega} d \omega \\
& =\sum_{j \in \mathbb{Z}} \frac{1}{2 \pi} \int_{\mathbb{R}} \widehat{f}(\omega) \widehat{\widehat{\psi}}\left(2^{-j} \omega\right) \widehat{\psi}^{*}\left(2^{-j} \omega\right) e^{i x \omega} d \omega \\
& =\frac{1}{2 \pi} \int_{\mathbb{R}} \widehat{f}(\omega) e^{i x \omega} d \omega=f(x) .
\end{aligned}
$$


ОПРЕДЕЛЕНИЕ 7.2. Функция $\widetilde{\psi} \in L^{2}(\mathbb{R})$ называется двоично-двойственной к двоичному всплеску $\psi$, если каждая функция $f \in L^{2}(\mathbb{R})$ может быть представлена в виде

$$
\begin{aligned}
f(x) & =\sum_{j \in \mathbb{Z}} \int_{\mathbb{R}}\left(W_{j}^{\psi} f\right)(b)\left\{2^{j} \widetilde{\psi}\left(2^{j}(x-b)\right)\right\} d b \\
& =\sum_{j \in \mathbb{Z}} 2^{3 j / 2} \int_{\mathbb{R}}\left(W_{\psi} f\right)\left(b, \frac{1}{2^{j}}\right) \widetilde{\psi}\left(2^{j}(x-b)\right) d b .
\end{aligned}
$$

В силу (7.2) функция $\psi^{*}$ является двоично-двойственной к $\psi$. Кроме того, $\psi^{*}$ является двоичньм всплеском:

$$
\frac{1}{B} \leqslant \sum_{j \in \mathbb{Z}}\left|\widehat{\psi}^{*}\left(2^{-j} \omega\right)\right|^{2} \leqslant \frac{1}{A}
$$

Отметим, что двоично-двойственньй всплеск к данному всплеску $\psi$ не единственен.

ТЕОрема 7.2. Пусть $\psi-$ двоичный всплеск и $\widetilde{\psi}-$ произвольная функция из $L^{2}(\mathbb{R})$, удовлетворяющая условию

$$
\left.\underset{x \in \mathbb{R}}{\operatorname{ess} \sup _{j \in \mathbb{Z}}} \sum_{j \in \widetilde{\psi}}\left(2^{-j} x\right)\right|^{2}<\infty \text {. }
$$

Функиия $\widetilde{\psi}$ является двоично-двойственной $\kappa \psi$ тогда и только тогда, когда

$$
\sum_{j \in \mathbb{Z}} \overline{\widehat{\psi}\left(2^{-j \omega}\right)} \widehat{\widetilde{\psi}}\left(2^{-j} \omega\right)=1 \quad \text { n.e. }
$$

8. Фреймы. Для вьгислительной эффективности можно дискретизировать и параметр сдвига $b$, рассматривая только дискретное множество значений: $b_{j, k}:=\frac{k}{2 j} b_{0}$, $j, k \in \mathbb{Z}$, где $b_{0}>0$ - фиксированная константа, называемая темпом измерений. Обозначим

$$
\psi_{b_{0}, j, k}(t):=\psi_{b_{j, k}, a_{j}}(t)=2^{j / 2} \psi\left(2^{j} t-k b_{0}\right) .
$$

Будем рассматривать только следующие значения ИВП:

$$
\left(W_{\psi} f\right)\left(b_{j, k}, a_{j}\right)=\left\langle f, \psi_{b_{0}, j, k}\right\rangle, \quad j, k \in \mathbb{Z}
$$

Если сушествуют константы $A$ и $B, 0<A \leqslant B<\infty$, такие, что

$$
A\|f\|_{2}^{2} \leqslant \sum_{j, k \in \mathbb{Z}}\left|\left\langle f, \psi_{b_{0}, j, k}\right\rangle\right|^{2} \leqslant B\|f\|_{2}^{2}, \quad f \in L^{2}(\mathbb{R}),
$$

то функщию $f \in L^{2}(\mathbb{R})$ можно восстановить по значениям ИВП из (8.1). 
ОПРЕДЕЛЕНИЕ 8.1. Говорят, что функция $\psi \in L^{2}(\mathbb{R})$ порождает фрейм в $L^{2}(\mathbb{R})$ с темпом измерений $b_{0}>0$, если вьполняется (8.2) с константами $A$ и $B$, которые называются границами фрейма. Если $A=B$, то фрейм называют жестким (естественно называть жесткий фрейм обобшенной системой Парсеваля).

Рассмотрим линейньй оператор $T$ на $L^{2}(\mathbb{R})$ :

$$
T f:=\sum_{j, k \in \mathbb{Z}}\left\langle f, \psi_{b_{0}, j, k},\right\rangle \psi_{b_{0}, j, k}, \quad f \in L^{2}(\mathbb{R})
$$

Из (8.2) следует, что Т является взаимно-однозначным. Действительно,

$$
\langle T f, f\rangle=\sum_{j, k \in \mathbb{Z}}\left|\left\langle f, \psi_{b_{0}, j, k}\right\rangle\right|^{2}
$$

Поэтому для $g=T f$ имеем

$$
A\left\|T^{-1} g\right\|_{2}^{2}=A\|f\|_{2}^{2} \leqslant\langle T f, f\rangle=\left\langle g, T^{-1} g\right\rangle \leqslant\|g\|_{2}\left\|T^{-1} g\right\|_{2} .
$$

Откуда $\left\|T^{-1} g\right\|_{2} \leqslant \frac{1}{A}\|g\|_{2}$ или $\left\|T^{-1}\right\| \leqslant A^{-1}$.

Таким образом, любую функцию $f \in L^{2}(\mathbb{R})$ можно восстановить по значениям ИВП из (8.1), применяя формулу

$$
f=T^{-1} T f=\sum_{j, k \in \mathbb{Z}}\left\langle f, \psi_{b_{0}, j, k}\right\rangle T^{-1} \psi_{b_{0}, j, k} .
$$

Полагая $\psi_{b_{0}}^{j, k}:=T^{-1} \psi_{b_{0}, j, k}, j, k \in \mathbb{Z}$, можно переписать формулу (8.3) следующим образом: для любых $f, g \in L^{2}(\mathbb{R})$

$$
\begin{aligned}
\langle f, g\rangle & =\sum_{j, k \in \mathbb{Z}}\left\langle f, \psi_{b_{0}, j, k}\right\rangle\left\langle\psi_{b_{0}}^{j, k}, g\right\rangle ; \\
f & =\sum_{j, k \in \mathbb{Z}}\left\langle f, \psi_{b_{0}, j, k}\right\rangle \psi_{b_{0}}^{j, k} .
\end{aligned}
$$

Естественно называть $\left\{\psi_{b_{0}}^{j, k}\right\}$ двойственньм фреймом к фрейму $\left\{\psi_{b_{0}, j, k}\right\}$.

ОПРЕДЕЛЕНИЕ 8.2. Говорят, что функция $\psi \in L^{2}(\mathbb{R})$ порождает базис Рисса (или безусловный базис) $\left\{\psi_{b_{0}, j, k}\right\}$ с темпом измерений $b_{0}$, если вьполнены следующие два условия:

(i) линейная оболочка $\left\{\psi_{b_{0}, j, k}\right\}_{j, k \in \mathbb{Z}}$ плотна в $L^{2}(\mathbb{R})$;

(ii) существуют положительные константы $A$ и $B, 0<A \leqslant B<\infty$, такие, что для любых $\left\{c_{j, k}\right\} \in l^{2}\left(\mathbb{Z}^{2}\right)$

$$
A\left\|\left\{c_{j, k}\right\}\right\|_{l^{2}}^{2} \leqslant\left\|\sum_{j, k \in \mathbb{Z}} c_{j, k} \psi_{b_{0}, j, k}\right\|_{2}^{2} \leqslant B\left\|\left\{c_{j, k}\right\}\right\|_{l^{2}}^{2} .
$$


Константы $A$ и $B$ назьвают константами Рисса для $\left\{\psi_{b_{0}, j, k}\right\}$. Если $b_{0}=1$, то функция $\psi$ назьвается $R$-функцией.

В дальнейшем будем использовать обозначение

$$
\psi_{j, k}(x):=\psi_{1, j, k}(x)=2^{j / 2} \psi\left(2^{j} x-k\right),
$$

которое не надо путать с $\psi_{b, a}$ из (6.1).

Следующий результат показывает разнищу между фреймом и базисом Рисса.

Tеорема 8.1. Пусть $\psi \in L^{2}(\mathbb{R})$ и $b_{0}>0$. Тогда следующие утверждения әквивалентны:

(i) $\left\{\psi_{b_{0}, j, k}\right\}-$ базис Pисса в $L^{2}(\mathbb{R})$;

(ii) $\left\{\psi_{b_{0}, j, k}\right\}$ - фрейм в $L^{2}(\mathbb{R})$ и $l^{2}$-линейно независимое семейство, т.е. если $\sum c_{j, k} \psi_{b_{0}, j, k}=0 u\left\{c_{j, k}\right\} \in l^{2}$, mo $c_{j, k}=0$.

Более того, константы Рисса совпадают с границами фрейма.

Функция, порождаюшая фрейм, всегда является двоичным всплеском.

Теорема 8.2. Пусть $\psi \in L^{2}(\mathbb{R})$ порожљдает фрейм $\left\{\psi_{b_{0}, j, k}\right\}$ в $L^{2}(\mathbb{R})$ с гранииами $A, B$ и темпом измерений $b_{0}>0$. Тогда

$$
b_{0} A \leqslant \sum_{j \in \mathbb{Z}}\left|\widehat{\psi}\left(2^{-j} \omega\right)\right|^{2} \leqslant b_{0} B \quad \text { n. } \boldsymbol{B} .
$$

9. Всплесковые ряды. В дальнейшем будем предполагать темпизмерений $b_{0}=1$. Пусть $\psi-R$-функция, $\left\{\psi_{j, k}\right\}$ - базис Рисса, $\left\{\psi^{j, k}=T^{-1} \psi_{j, k}\right\}$ - двойственный фрейм (см. (8.3)).

В классе $R$-функций выделяют два важных подмножества.

ОПРеДЕЛЕниЕ 9.1. Пусть $\psi \in L^{2}(\mathbb{R})$ есть $R$-функция. Тогда

(i) $\psi$ назьвают ортогональным всплеском, если $\psi_{j, k}$ удовлетворяют условию ортогональности:

$$
\left\langle\psi_{j, k}, \psi_{l, m}\right\rangle=\delta_{j, l} \delta_{k, m}, \quad j, k, l, m \in \mathbb{Z} ;
$$

(ii) $\psi$ называют полуортогональньм всплеском, если $\psi_{j, k}$ удовлетворяют условию:

$$
\left\langle\psi_{j, k}, \psi_{l, m}\right\rangle=0, \quad j \neq l ; \quad j, k, l, m \in \mathbb{Z} .
$$

Очевидно, что ортогональные всплески являются самодвойственными: $\psi^{j, k}=\psi_{j, k}$, $j, k \in \mathbb{Z}$.

Для того чтобы указать двойственньй фрейм в полуортогональном случае, приведем следующий критерий ортогональности. 
ТЕОрема 9.1. Для любой функиии $\phi \in L^{2}(\mathbb{R})$ следующие утверэкдения әквивалентны.

(i) $\{\phi(x-k): k \in \mathbb{Z}\}$ - ортонормированное семейство:

$$
\langle\phi(\cdot-k), \phi(\cdot-l)\rangle=\delta_{k, l}, \quad k, l \in \mathbb{Z} .
$$

(ii) Преобразование Фурье $\widehat{\phi}$ удовлетворяет условию:

$$
\frac{1}{2 \pi} \int_{\mathbb{R}} e^{-i j x}|\widehat{\phi}(x)|^{2} d x=\delta_{j, 0}, \quad j \in \mathbb{Z}
$$

(iii) Для почти всех $x$

$$
\sum_{j \in \mathbb{Z}}|\widehat{\phi}(x+2 \pi k)|^{2}=1
$$

Более слабым, чем условие ортогональности, является условие Рисса, или условие безусловности.

ТеОРема 9.2. Для любой функиии ф и констант $0<A \leqslant B<\infty$ следующие утверждения әквивалентны.

(i) $\{\phi(\cdot-k): k \in \mathbb{Z}\}$ удовлетворяет условию Рисса с константами $A$ и $B$, m.e. для любых $\left\{c_{k}\right\} \in l^{2}$

$$
A\left\|\left\{c_{k}\right\}\right\|_{l^{2}}^{2} \leqslant\left\|\sum_{k \in \mathbb{Z}} c_{k} \phi(\cdot-k)\right\|_{2}^{2} \leqslant B\left\|\left\{c_{k}\right\}\right\|_{l^{2}}^{2} .
$$

(ii) Преобразование Фурье $\widehat{\phi}$ удовлетворяет п.в. условию

$$
A \leqslant \sum_{k \in \mathbb{Z}}|\widehat{\phi}(x+2 \pi k)|^{2} \leqslant B .
$$

ТеОРема 9.3. Пусть $\psi \in L^{2}(\mathbb{R})$ - полуортогональный всплеск. Определим $\widetilde{\psi}$ в образах Фурье

$$
\widehat{\widetilde{\psi}}(\omega):=\frac{\widehat{\psi}(\omega)}{\sum_{k \in \mathbb{Z}}|\widehat{\psi}(\omega+2 \pi k)|^{2}} .
$$

Тогда функиия $\widetilde{\psi}$ двойственна к $\psi$, т.е.

$$
\left\langle\psi_{j, k}, \widetilde{\psi}_{l, m}\right\rangle=\delta_{j, l} \delta_{k, m}, \quad j, k, l, m \in \mathbb{Z},
$$

где $\widetilde{\psi}_{l, m}(x):=2^{l / 2} \widetilde{\psi}\left(2^{l} x-m\right)$. Таким образом, двойственныи фрейм $\kappa\left\{\psi_{j, k}\right\}$ это $\left\{\psi^{j, k}=\widetilde{\psi}_{j, k}\right\}$.

Эта теорема указьвает, как исправить полуортогональный всплеск в ортогональньй. Действительно, полагая

$$
\widehat{\psi^{\perp}}(\omega):=\frac{\widehat{\psi}(\omega)}{\left(\sum_{k \in \mathbb{Z}}|\widehat{\psi}(\omega+2 \pi k)|^{2}\right)^{1 / 2}},
$$


получаем, что

$$
\widehat{\widehat{\psi^{\perp}}}(\omega)=\frac{\widehat{\psi^{\perp}}(\omega)}{\left.\sum_{k \in \mathbb{Z}} \widehat{\mid \psi^{\perp}}(\omega+2 \pi k)\right|^{2}}=\widehat{\psi^{\perp}}(\omega) \text {. }
$$

Таким образом, $\widetilde{\psi^{\perp}}=\psi^{\perp}$, т.е. $\psi^{\perp}-$ самодвойственен.

Существуют $R$-функции, у которых нет двойственных, т.е. двойственньй базис $\left\{\psi^{j, k}\right\}$ к базису Рисса $\left\{\psi_{j, k}\right\}$ не имеет вида $\left\{\widetilde{\psi}_{j, k}\right\}$ ни для какой функции $\widetilde{\psi} \in L^{2}(\mathbb{R})$.

ОПреДЕлЕниЕ 9.2. $R$-функшия $\psi \in L^{2}(\mathbb{R})$ назьвается всплеском, если существует двойственная функция $\widetilde{\psi} \in L^{2}(\mathbb{R})$ такая, что $\left\{\psi_{j, k}\right\}$ и $\left\{\widetilde{\psi}_{j, k}\right\}$ удовлетворяют (9.1).

Очевидно, что $\widetilde{\psi}$ - тоже всплеск с двойственным $\psi$.

Если $\psi$ - всплеск с двойственным $\widetilde{\psi}$, то любую функцию $f \in L^{2}(\mathbb{R})$ можно разложить в ряды:

$$
f(x)=\sum_{j, k \in \mathbb{Z}} c_{j, k} \psi_{j, k}=\sum_{j, k \in \mathbb{Z}} d_{j, k} \widetilde{\psi}_{j, k}
$$

Оба этих ряда называются всплесковыми. В силу (9.1)

$$
c_{j, k}=\left\langle f, \widetilde{\psi}_{j, k}\right\rangle ; \quad d_{j, k}=\left\langle f, \psi_{j, k}\right\rangle
$$

ТЕОРЕМа 9.4. Пусть $\psi-$ всплеск с двойственным $\widetilde{\psi}$. Для произвольной функиии $f \in L^{2}(\mathbb{R})$ вычислим ИВП с ни $\widetilde{\psi}$ в точках $(b, a)=\left(\frac{k}{2^{j}}, \frac{1}{2^{j}}\right), j, k \in \mathbb{Z}$ :

$$
d_{j, k}=\left\langle f, \psi_{j, k}\right\rangle=\left(W_{\psi} f\right)\left(\frac{k}{2^{j}}, \frac{1}{2^{j}}\right) ; \quad c_{j, k}=\left\langle f, \widetilde{\psi}_{j, k}\right\rangle=\left(W_{\widetilde{\psi}} f\right)\left(\frac{k}{2^{j}}, \frac{1}{2^{j}}\right) .
$$

Тогда $f$ можсно восстановить или по $\left\{d_{j, k}\right\}$, или по $\left\{c_{j, k}\right\}$, используя рядь (9.3). Более того, скалярное произведение любых двух функций из $L^{2}(\mathbb{R})$ моэмно также вычислить при помощи дискретных значений ИВП:

$$
\langle f, g\rangle=\sum_{j, k}\left\langle f, \psi_{j, k}\right\rangle\left\langle\widetilde{\psi}_{j, k}, g\right\rangle .
$$

10. Система Хаара на прямой. Система Хаара на всей прямой является самым простым, но вместе с тем и одним из самых модельных примеров ортогональных всплесков. Мы расскажем о ней с современных позиций теории всплесков, подготавливая читателей к пониманию общей схемы построения всплесков, так называемому кратномасштабному анализу (multiresolution analysis).

Пусть $\varphi^{H}(t)=\kappa_{[0,1]}(t)$ (в современной терминологии, это - масштабируюшая функция Хаара). Рассмотрим замыкание по норме $L^{2}(\mathbb{R})$ линейной оболочки целочисленных сдвигов функции $\varphi^{H}$ :

$$
V_{0}:=\left[\varphi_{0 k}^{H}(\cdot):=\varphi^{H}(\cdot-k)\right]_{k \in \mathbb{Z}}=\left\{\sum_{k \in \mathbb{Z}} c_{0 k} \varphi_{0 k}^{H}: \sum_{k \in \mathbb{Z}}\left|c_{0 k}\right|^{2}<\infty\right\} .
$$


Естественно назвать это подпространство подпространством функций масштаба 1. Для анализа функций из $L^{2}(\mathbb{R})$ нужны подпространства функций с различньми масштабами. Определим последовательность подпространств $\left\{V_{j}\right\}_{j \in \mathbb{Z}}: V_{j}:=$ $\left[\varphi_{j k}^{H}(t):=2^{j / 2} \varphi^{H}\left(2^{j} t-k\right)\right]_{k \in \mathbb{Z}}\left(V_{j}-\right.$ подпространство функций масштаба $\left.2^{-j}\right)$. Отметим, что последовательность $\left\{\varphi_{j k}^{H}\right\}_{k \in \mathbb{Z}}$ образует ортонормированный базис в $V_{j}$. Очевидно, что

$$
\bigcap_{j \in \mathbb{Z}} V_{j}=\{0\}
$$

и

$$
\overline{\bigcup_{j \in \mathbb{Z}} V_{j}}=L^{2}(\mathbb{R}) .
$$

Здесь $\bar{X}$ обозначает замыкание подпространства $X$ по норме $L^{2}(\mathbb{R})$.

Последнее свойство наталкивает на мысль получить ортонормированный базис в $L^{2}(\mathbb{R})$, используя совокупность ортонормированных базисов в $V_{j}$. На этом пути есть небольшое препятствие. Несмотря на вложение $V_{j} \subseteq V_{j+1}$, ортонормированньй базис $\left\{\varphi_{j k}^{H}\right\}_{k \in \mathbb{Z}}$ в $V_{j}$ не является частью ортонормированного базиса $\left\{\varphi_{j+1, k}^{H}\right\}_{k \in \mathbb{Z}}$ в $V_{j+1}$. Поэтому необходимо рассуждать следуюшим образом. Пусть $W_{0}-$ это ортогональное дополнение $V_{0}$ до $V_{1}: V_{0} \oplus W_{0}=V_{1}$. Базис пространства $V_{0}$ состоит из целочисленных сдвигов функции $\varphi_{00}^{H}$. Базис пространства $V_{1}$ состоит из сдвигов на $k / 2(k \in \mathbb{Z})$ функции $\varphi_{1}^{H}(t)=\sqrt{2} \varphi(2 t): \varphi_{1, k}^{H}(t)=\varphi_{1}^{H}(t-k / 2)$. В силу этих фактов естественно попытаться найти функцию $\psi$, целочисленные сдвиги которой образуют ортонормированный базис в $W_{0}$. Таким свойством обладает функция

$$
\psi^{H}(t)= \begin{cases}1, & t \in\left(0, \frac{1}{2}\right) \\ -1, & t \in\left(\frac{1}{2}, 1\right) \\ 0 & \text { в остальных случаях. }\end{cases}
$$

Это и есть всплеск Хаapa.

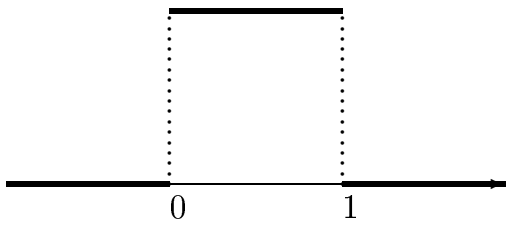

Рис. 8. Масштабирующая функция Хаара

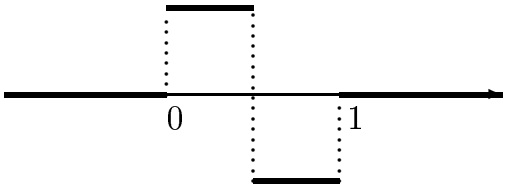

Рис. 9. Всплеск Хаара

Итак, $W_{0}=\left[\psi_{0 k}^{H}(\cdot):=\psi^{H}(\cdot-k)\right]_{k \in \mathbb{Z}} \cdot$ Если определить $W_{j}:=\left[\psi_{j k}^{H}(t):=\right.$ $\left.2^{j / 2} \psi^{H}\left(2^{j} t-k\right)\right]_{k \in \mathbb{Z}}$, то очевидно, что

$$
V_{j} \oplus W_{j}=V_{j+1}
$$


Из (10.1)-(10.3) следует, что

$$
L^{2}(\mathbb{R})=\bigoplus_{j=-\infty}^{\infty} W_{j}
$$

Поскольку пространства $W_{j}$ взаимно ортогональны, то, объединяя все ортонормиро-


Отметим сразу, что в приложениях чаще всего удобнее заменить в $(10.4) \bigoplus_{j=-\infty}^{-1} W_{j}$ на $V_{0}: V_{0} \oplus\left\{\bigoplus_{j=0}^{\infty} W_{j}\right\}=L^{2}(\mathbb{R})$. В этом случае ортонормированный базис в $L^{2}(\mathbb{R})$



Таким образом, любую функцию из $L^{2}(\mathbb{R})$ можно разложить в ряд

$$
f=\sum_{j \in \mathbb{Z}} \sum_{k \in \mathbb{Z}} d_{j k} \psi_{j k}^{H}=\sum_{k \in \mathbb{Z}} c_{0 k} \varphi_{0 k}^{H}+\sum_{j=0}^{\infty} \sum_{k \in \mathbb{Z}} d_{j k} \psi_{j k}^{H}
$$

Последний ряд удобен, в частности, потому, что легко переносится со всей прямой на отрезок $[0,1]$. Для $f \in L^{2}[0,1]$ имеем

$$
f=c_{0}+\sum_{j=0}^{\infty} \sum_{k=0}^{2^{j}-1} d_{j k} \psi_{j k}^{H} .
$$

Именно в таком виде систему определил А. Хаар [16].

Перечислим преимущества (10.5) по сравнению с классическим рядом Фурье по тригонометрической системе

$$
f(t)=\frac{1}{2} a_{0}+\sum_{l=1}^{\infty}\left(a_{l} \cos l t+b_{l} \sin l t\right)
$$

Первое преимушество состоит в том, что ряд Хаара является хорошо локализованньм. Если мы интересуемся поведением функции $f$ на подынтервале $[a, b]$, то в разложении (10.5) нам нужны только те индексы $j$ и $k$, для которых $\operatorname{supp} \psi_{j k}^{H}=\left[k 2^{-j},(k+1) 2^{-j}\right]$ пересекается с $[a, b]$, тогда как в разложении $(10.6)$ нам потребуются все коэффициенты. Второе отличие состоит в том, что частичная сумма ряда Хаара по $j=0,1,2, \ldots, N$ является приближением исходной функции с точностью до масштаба $2^{-N-1}$. Эти два свойства, локализованность и масштабирование, являются характерными для всех всплесковых разложений.

Прежде чем рассказать о других всплесках, изложим наиболее общий метод построения всплесков, так назьваемьй кратномасштабньй анализ. 
11. Кратномасштабный анализ в $L^{2}(\mathbb{R})$.

ОПРЕДЕЛЕнИЕ 11.1. Кратномасштабньй анализ (КМА) - это последовательность $\left\{V_{j}\right\}_{j \in \mathbb{Z}}$ замкнутых подпространств $L^{2}(\mathbb{R})$, удовлетворяюшая следуюшим свойствам:

$$
\begin{gathered}
V_{j} \subset V_{j+1} ; \\
\bigcap_{j \in \mathbb{Z}} V_{j}=L^{2}(\mathbb{R}) ; \\
f \in V_{j} \Leftrightarrow f\left(2^{-j} \cdot\right) \in V_{0} ; \\
f \in V_{0} \Leftrightarrow f(\cdot-k) \in V_{0} \text { для любого } k \in \mathbb{Z} ;
\end{gathered}
$$

существует функция $\varphi \in V_{0}$ такая, что последовательность

$$
\{\varphi(\cdot-k)\}_{k \in \mathbb{Z}} \text { образует базис Рисса в } V_{0} .
$$

Это понятие введено и исследовано в [17].

Если обозначить через $P_{j}$ ортогональный проектор на $V_{j}$, то из условия $(11.2)$ следует, что $\lim _{j \rightarrow \infty} P_{j} f=f$ для любой функции $f \in L^{2}(\mathbb{R})$. Условие (11.4) означает, что все подпространства $V_{j}$ однозначно определяются из центрального подпространства $V_{0}$ при помощи соответствующей замены переменных (соответствующего изменения масштаба). Из (11.4) и (11.5) следует, что для любой функции $f \in V_{j}$ функция $f\left(\cdot-2^{-j} k\right)$ также принадлежит $V_{j}$ при любом $k \in \mathbb{Z}$. Пусть $\varphi_{j k}(t):=2^{j / 2} \varphi\left(2^{j} t-k\right)$; $j, k \in \mathbb{Z}$. Из (11.4) и (11.6) следует, что последовательность $\left\{\varphi_{j k}\right\}_{k \in \mathbb{Z}}$ является базисом Рисса в $V_{j}$ для любого $j \in \mathbb{Z}$. Не ограничивая обшности, можно считать, что $\{\varphi(\cdot-k)\}_{\mathbb{Z}}-$ ОНБ в $V_{0}$ (этого всегда можно достичь за счет ортогонализации (9.2)).

Основньм свойством КМА является возможность построения ортонормированного всплескового базиса $\left\{\psi_{j k}\right\}_{j, k \in \mathbb{Z}}, \psi_{j k}(t)=2^{j / 2} \psi\left(2^{j} t-k\right)$, такого, что для любой функции $f$ из $L^{2}(\mathbb{R})$

$$
P_{j+1} f=P_{j} f+\sum_{k \in \mathbb{Z}}\left\langle f, \psi_{j k}\right\rangle \psi_{j k}
$$

Опишем процесс построения такого базиса. Пусть $W_{j}$ - это ортогональное дополнение $V_{j}$ до $V_{j+1}: W_{j} \oplus V_{j}=V_{j+1}$. В силу (11.1)

$$
W_{j} \perp W_{j_{1}} \text { при } j \neq j_{1},
$$

и при любых $j_{0}<j$

$$
V_{j}=V_{j_{0}} \oplus\left(\bigoplus_{l=j_{0}}^{j-1} W_{l}\right) .
$$

Из (11.2) и (11.3) следует, что

$$
L^{2}(\mathbb{R})=\bigoplus_{j \in \mathbb{Z}} W_{j}
$$




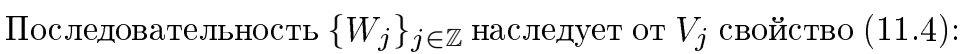

$$
f \in W_{j} \Longleftrightarrow f\left(2^{-j} \cdot\right) \in W_{0} .
$$

Формула (11.7) эквивалентна тому, что при фиксированном $j$ последовательность $\left\{\psi_{j k}\right\}_{k \in \mathbb{Z}}$ образует ортонормированньй базис в $W_{j}$. В силу (11.10) последнее означает, что $\left\{\psi_{j k}\right\}_{j, k \in \mathbb{Z}}$ - ортонормированный базис в $L^{2}(\mathbb{R})$. Заметим теперь, что свойство (11.11) гарантирует, что $\left\{\psi_{j k}\right\}_{k \in \mathbb{Z}}$ будет базисом в $W_{j}$, если $\left\{\psi_{0 k}\right\}_{k \in \mathbb{Z}}$ является базисом в $W_{0}$. Таким образом, задача построения всплескового базиса со свойством (11.7) сводится к нахождению функции $\psi$ такой, что последовательность $\{\psi(\cdot-k)\}_{k \in \mathbb{Z}}$ образует ортонормированньй базис в $W_{0}$.

Для построения функции $\psi$ нам потребуются некоторые свойства $\varphi$ и $W_{0}$. Так как $\varphi \in V_{0} \subset V_{1}$ и $\left\{\varphi_{1 k}\right\}_{k \in \mathbb{Z}}-$ ортонормированный базис в $V_{1}$, то

$$
\varphi=\sum_{k \in \mathbb{Z}} h_{k} \varphi_{1 k}
$$

где

$$
h_{k}:=\left\langle\varphi, \varphi_{1 k}\right\rangle, \quad \sum_{k \in \mathbb{Z}}\left|h_{k}\right|^{2}=1
$$

Переходя к образам Фурье, имеем

$$
\widehat{\varphi}(\omega)=m(\omega / 2) \widehat{\varphi}(\omega / 2),
$$

где $m(\omega)=\frac{1}{\sqrt{2}} \sum_{k \in \mathbb{Z}} h_{k} e^{-i k \omega}$. Функцию $\varphi$ называют масштабируюшей (scaling), равенство (11.12) - масштабньм, равенство (11.14) - уточняюшим (refinement), функцию $m$ - уточняюшей маской (refinement mask) или масштабируюшим фильтром (scaling filter).

В силу теоремы 9.1

$$
\sum_{l \in \mathbb{Z}}|\widehat{\varphi}(\omega+2 \pi l)|^{2}=1
$$

для почти всех $\omega$. Если подставить (11.14) в (11.15), то получим, что

$$
\sum_{l \in \mathbb{Z}}|m(\omega / 2+\pi l) \widehat{\varphi}(\omega / 2+\pi l)|^{2}=1 .
$$

Разбивая сумму на две (по четньм и по нечетным $l$ ) и учитьвая $2 \pi$-периодичность $m$, имеем

$$
\begin{gathered}
\sum_{l \in \mathbb{Z}}|m(\omega / 2+2 \pi l) \widehat{\varphi}(\omega / 2+2 \pi l)|^{2}+\sum_{l \in \mathbb{Z}}|m(\omega / 2+2 \pi l+\pi) \widehat{\varphi}(\omega / 2+2 \pi l+\pi)|^{2} \\
=|m(\omega / 2)|^{2}+|m(\omega / 2+\pi)|^{2}=1 .
\end{gathered}
$$


Охарактеризуем подпространство $W_{0}$ в терминах образов Фурье. Любая функция $f$ из $W_{0}$ принадлежит $V_{1}$ и ортогональна $V_{0}$. Первое свойство означает, что $f=$ $\sum_{k \in \mathbb{Z}} f_{k} \varphi_{1 k}$, где $f_{k}=\left\langle f, \varphi_{1 k}\right\rangle$. В образах Фурье имеем

$$
\widehat{f}(\omega)=m_{f}(\omega / 2) \widehat{\varphi}(\omega / 2),
$$

где $m_{f}=\frac{1}{\sqrt{2}} \sum_{k \in \mathbb{Z}} f_{k} e^{-i k \omega}-2 \pi$-периодическая функция из $L^{2}[0,2 \pi]$. Условие ортогональности $f$ к $V_{0}$ эквивалентно тому, что $f \perp \varphi_{0 k}$ для любого $k \in \mathbb{Z}$, т.е.

$$
\int_{\mathbb{R}} \widehat{f}(\omega) \overline{\hat{\varphi}(\omega)} e^{i k \omega} d \omega=0
$$

Заметим, что

$$
\int_{\mathbb{R}} \widehat{f}(\omega) \overline{\hat{\varphi}(\omega)} e^{i k \omega} d \omega=\int_{0}^{2 \pi} e^{i k \omega} \sum_{l \in \mathbb{Z}} \widehat{f}(\omega+2 \pi l) \widehat{\hat{\varphi}}(\omega+2 \pi l) d \omega=0 .
$$

Так как равенство (11.18) имеет место для любого $k \in \mathbb{Z}$, то

$$
\sum_{l \in \mathbb{Z}} \widehat{f}(\omega+2 \pi l) \bar{\varphi}(\omega+2 \pi l)=0 .
$$

Ряд в (11.19) сходится абсолютно в $L^{1}([0,2 \pi])$. Подставляя $(11.14)$ и $(11.17)$ в (11.19) и группируя суммы с четными и нечетными $l$, получим, учитывая $(11.15)$, что

$$
\sum_{l \in \mathbb{Z}} \widehat{f}(\omega+2 \pi l) \bar{\varphi}(\omega+2 \pi l)=m_{f}(\omega / 2) \bar{m}(\omega / 2)+m_{f}(\omega / 2+\pi) \bar{m}(\omega / 2+\pi)=0 .
$$

В силу $(11.16) \bar{m}(\omega)$ и $\bar{m}(\omega+\pi)$ не могут обратиться в нуль одновременно, поэтому сушествует $2 \pi$-периодическая функция $\lambda(\omega)$ такая, что

$$
m_{f}(\omega)=\lambda(\omega) \bar{m}(\omega+\pi) \text { п.в. }
$$

и $\lambda(\omega)+\lambda(\omega+\pi)=0$. Последнее равенство можно переписать как $\lambda(\omega)=e^{-i \omega} \nu(2 \omega)$, где $\nu$ - некоторая $2 \pi$-периодическая функция. Таким образом, преобразование Фурье произвольной функции из $W_{0}$ имеет вид

$$
\widehat{f}(\omega)=e^{-i \omega / 2} \bar{m}(\omega / 2+\pi) \nu(\omega) \widehat{\varphi}(\omega / 2),
$$

где $\nu$ - некоторая $2 \pi$-периодическая функция. Кроме того,

$$
\begin{aligned}
\|f\|_{L^{2}(\mathbb{R})} & =\frac{1}{2 \pi}\|\widehat{f}\|_{L^{2}(\mathbb{R})} \\
& =\frac{1}{2 \pi} \int_{0}^{2 \pi}|\nu|^{2} \sum_{l \in \mathbb{Z}}|m(\omega / 2+\pi l+\pi) \widehat{\varphi}(\omega / 2+\pi l)|^{2} d \omega \\
& =\|\nu\|_{L^{2}([0,2 \pi])} .
\end{aligned}
$$

Таким образом, $2 \pi$-периодическая функция $\nu$ является квадратично суммируемой. 
Имея описание (11.21) пространства $W_{0}$, легко найти функцию $\psi \in W_{0}$, целые сдвиги которой $\{\psi(\cdot-k)\}_{k \in \mathbb{Z}}$ образуют ортонормированный базис в $W_{0}$. Пусть $\psi$ - искомая функция. Тогда $\widehat{\psi}(\omega)=e^{-i \omega / 2} \bar{m}(\omega / 2+\pi) \nu_{\psi}(\omega) \widehat{\varphi}(\omega / 2)$. Подставляя это выражение в (11.15), получаем, используя (11.16), что

$$
\left|\nu_{\psi}(\omega)\right|^{2} \equiv 1 \text { п.в. }
$$

Проще всего положить $\nu_{\psi}(\omega) \equiv 1$. В силу (11.16) целые сдвиги функции $\psi$, определяемой равенством

$$
\widehat{\psi}(\omega)=e^{-i \omega / 2} \bar{m}(\omega / 2+\pi) \widehat{\varphi}(\omega / 2),
$$

будут базисом в $W_{0}$.

Действительно, если $\nu(\omega)=\sum_{k \in \mathbb{Z}} \nu_{k} e^{-i k \omega}$, то $\widehat{f}(\omega)=\left(\sum_{k \in \mathbb{Z}} \nu_{k} e^{-i k \omega}\right) \widehat{\psi}(\omega)$ или $f(\cdot)=\sum_{k \in \mathbb{Z}} \nu_{k} \psi(\cdot-k)$.

Заметим, что в силу (11.22) образ Фурье любой функции, целые сдвиги которой образуют ортонормированньй базис в $W_{0}$, отличается от образа Фурье функции $\psi$ из (11.23) лишь некоторым $2 \pi$-периодическим множителем, по модулю равным 1.

Таким образом, имея кратномасштабный анализ $\left\{V_{j}\right\}_{j \in \mathbb{Z}}$, порождаемый масштабируюшей функцией $\varphi$, всегда можно построить ортонормированный всплесковый базис $\left\{\psi_{j k}\right\}_{j, k \in \mathbb{Z}}$ в $L^{2}(\mathbb{R})$, обладающий свойством (11.7).

Из формулы (11.23) следует, что

$$
\psi(t)=\sum_{k \in \mathbb{Z}}(-1)^{k-1} h_{-k+1} \varphi_{1 k}(t) .
$$

В литературе для сокращения записей чаще всего используют

$$
\psi(t)=\sum_{k \in \mathbb{Z}}(-1)^{k} h_{-k+1} \varphi_{1 k}(t) .
$$

В заключение этого раздела проанализируем, более подробно свойства (11.2) и (11.3). Приводимые ниже результаты доказаны в [18].

Пусть $\widehat{V}:=\{\widehat{f}: f \in V\}$. Из тождества Планшереля и свойств (11.4)-(11.6) следует, что $f \in V_{j}$ тогда и только тогда, когда

$$
\widehat{f}(\omega)=m\left(\xi 2^{-j}\right) \widehat{\varphi}\left(\omega 2^{-j}\right), \quad \widehat{f}(\omega) \in L_{2}(\mathbb{R}),
$$

где $m$ - некоторая $2 \pi$-периодическая функция.

Лемма 11.1. Из свойств (11.4) и (11.5) следует, что пространство $\overline{\bigcup_{j \in \mathbb{Z}} V_{j}}$ инвариантно относительно сдвигов. 
ДокаЗАтельство. В силу (11.4), (11.5) из $f \in \bigcup_{j \in \mathbb{Z}} V_{j}$ следует, что $f(\cdot+t) \in$ $\bigcup_{j \in \mathbb{Z}} V_{j}$ для любого двоично-рационального $t=2^{-j} l, l, k \in \mathbb{Z}$. Так как сдвиг является непрерывной операцией в $L_{2}(\mathbb{R})$, то $f(\cdot+t) \in \overline{\bigcup_{j \in \mathbb{Z}} V_{j}}$ для любого $t \in \mathbb{R}$. Если теперь $g \in \overline{\bigcup_{j \in \mathbb{Z}} V_{j}}$, то, приближая $g$ функциями $f \in \bigcup_{j \in \mathbb{Z}} V_{j}$ и замечая, что $\|f(\cdot+t)-g(\cdot+t)\|_{L_{2}(\mathbb{R})}=\|g-f\|_{L_{2}(\mathbb{R})}$, получаем утверждение леммы.

Хорошо известно, что замкнутое подпространство $X$ в $L_{2}(\mathbb{R})$ является инвариантным относительно сдвигов тогда и только тогда, когда $\widehat{X}=L_{2}(\Omega)$ для некоторого измеримого множества $\Omega$.

В дальнейшем равенства между множествами понимаются с точностью до множеств нулевой меры.

Теорема 11.1. Пусть последовательность $\left\{V_{j}\right\}_{j \in \mathbb{Z}}$ удовлетворяет свойствам (11.4)-(11.6). Тогда $\overline{\bigcup_{j \in \mathbb{Z}} V_{j}}=L_{2}(\mathbb{R})$ тогда и только тогда, когда $\Omega_{0}:=$ $\bigcup_{j \in \mathbb{Z}} \operatorname{supp} \hat{\varphi}\left(2^{-j} \cdot\right)=\mathbb{R}$.

Доказательство. Пусть $X:=\overline{\bigcup_{j \in \mathbb{Z}} V_{j}}$. Тогда $\widehat{X}=L_{2}(\Omega)$. Таким образом, $X=L_{2}(\mathbb{R})$ тогда и только тогда, когда $\Omega=\mathbb{R}$. Так как $\varphi\left(2^{j} \cdot\right) \in V_{j}, j \in \mathbb{Z}$, то $\operatorname{supp} \hat{\varphi}\left(2^{-j}.\right) \subset \Omega$. Поэтому $\Omega_{0} \subset \Omega$. Предположим теперь, что $\Omega \backslash \Omega_{0}$ содержит множество положительной меры $\Omega_{1}$. В силу (11.26) преобразование Фурье любого элемента из $V_{j}$ обнуляется на $\Omega_{1}$. Следовательно, то же самое верно для любого элемента из $\bigcup_{j \in \mathbb{Z}} V_{j}$. Переходя к пределу, получаем, что преобразование Фурье любого элемента из $X$ обнуляется на $\Omega_{1}$, что противоречит тому, что $\widehat{X} \supset L_{2}\left(\Omega_{1}\right)$.

СлЕДСТвИЕ 11.1. Пусть последовательность $\left\{V_{j}\right\}_{j \in \mathbb{Z}}$ удовлетворяет свойствам (11.4)-(11.6) и $\widehat{\varphi}$ не равно нулю почти всюду в некоторой окрестности нуля. Тогда $\overline{\bigcup_{j \in \mathbb{Z}} V_{j}}=L_{2}(\mathbb{R})$.

Докажем теперь, что условие (11.3) следует из свойств (11.4)-(11.6) кратномасштабного анализа. Для доказательства нам потребуются две леммы, первая из которых хорошо известна.

ЛЕмма 11.2. Пусть $\Omega$ - измеримое подмножество $\mathbb{R}$, причем $\Omega+\alpha t=\Omega$ для некоторого действительного числа $\alpha \neq 0$ и для любого двоично-рачионального числа $t \in \mathbb{R}$. Тогда $\Omega=\mathbb{R}$ или $\Omega=\varnothing$.

Кроме того, если $f$ - некоторая измеримая функиия на $\mathbb{R}$, удовлетворяющая условию $f(\cdot+\alpha t)=f(\cdot)$ для любого двоично-рачионального $t$, mо $f=$ const почти всюду.

Доказательство этой леммы основано на свойствах точек Лебега измеримой функшии.

Лемма 11.3. Пусть последовательность $\left\{V_{j}\right\}_{j \in \mathbb{Z}}$ замкнутых подпространств $L_{2}(\mathbb{R})$ удовлетворяет свойствам (11.4)-(11.6) кратномасштабного анализа. Тогда $Y=\bigcap_{j \in \mathbb{Z}} V_{j}$ имеет размерность $\leqslant 1$.

ДокАЗАТЕльство. Предположим, что $Y \neq\{0\}$. Докажем, что в этом случае $\operatorname{dim} Y=1$. Пусть $f, g$ - две произвольные функции из $Y$. Рассмотрим отображение 
$F: \mathbb{R} \rightarrow \mathbb{C}^{2}:$

$$
F(\omega)= \begin{cases}0, & \text { если } \widehat{f}(\omega)=\widehat{g}(\omega)=0, \\ \frac{(\widehat{f}(\omega), \widehat{g}(\omega))}{\widehat{f}(\omega)}, & \text { если } \widehat{f}(\omega) \neq 0, \widehat{g}(\omega)=0, \\ \frac{(\widehat{f}(\omega), \widehat{g}(\omega))}{\widehat{g}(\omega)}, & \text { если } \widehat{g}(\omega) \neq 0 .\end{cases}
$$

Докажем, что отображение $F$ постоянно на своем носителе. Для этого рассмотрим произвольное измеримое подмножество $K \subset \mathbb{C}^{2} \backslash\{0\}$. Предположим, что $A:=F^{-1}(K)$ имеет положительную меру. Пусть $D$ - это множество точек вида $2^{j} k \pi, k \in \mathbb{Z}, j \in \mathbb{Z}$. Рассмотрим $\omega \in A$ и $t=2^{j+1} k \pi \in D$. В силу (11.26) сушествуют $2 \pi$-периодические функции $\tau$ и $\nu$ такие, что $\widehat{f}(\omega)=\tau\left(\omega 2^{-j}\right) \widehat{\varphi}\left(\omega 2^{-j}\right), \widehat{g}(\omega)=\nu\left(\omega 2^{-j}\right) \widehat{\varphi}\left(\omega 2^{-j}\right)$ п.в. Так как $0 \notin K$, то $F(\omega) \neq 0$ и, значит, $\widehat{\varphi}\left(\omega 2^{-j}\right) \neq 0$. Поэтому

$$
\begin{aligned}
(\widehat{f}(\omega+t), \widehat{g}(\omega+t)) & =\widehat{\varphi}\left(2^{-j}(\omega+t)\right)\left(\tau\left(2^{-j} \omega+2 k \pi\right), \nu\left(2^{-j} \omega+2 k \pi\right)\right) \\
& =\widehat{\varphi}\left(2^{-j}(\omega+t)\right)\left(\tau\left(2^{-j} \omega\right), \nu\left(2^{-j} \omega\right)\right) \\
& =\frac{\widehat{\varphi}\left(2^{-j}(\omega+t)\right)}{\widehat{\varphi}\left(2^{-j} \omega\right)}(\widehat{f}(\omega), \widehat{g}(\omega)) .
\end{aligned}
$$

Полученное равенство означает, что либо $F(\omega+t)=0$, либо $F(\omega+t)=F(\omega)$. Поэтому $F(A+D) \subset K \cup\{0\}$. В силу леммы $11.2 A+D=\mathbb{R}$, так как $A$ имеет положительную меру. Таким образом, все ненулевые значения $F$ принадлежат $K$. Поскольку $K$ можно выбрать сколь угодно малым, отображение $F$ постоянно на своем носителе. Последнее означает, что функции $f$ и $g$ линейно зависимы.

Теорема 11.2. Пусть последовательность $\left\{V_{j}\right\}_{j \in \mathbb{Z}}$ замкнутых подпространств $L_{2}(\mathbb{R})$ удовлетворяет свойствам (11.4)-(11.6) кратномасштабного анализа. Тогда $Y:=\bigcap_{j \in \mathbb{Z}} V_{j}=\{0\}$.

ДокАЗАТЕльство. Предположим противное: пусть $f \in Y$ и $f \neq 0$. В силу (11.4) $V_{j}$ является сжатием в два раза $V_{j-1}$, поэтому подпространство $Y$ инварианто относительно сжатия в 2 раза. С другой стороны, в силу леммы $11.3 \operatorname{dim} Y \leqslant 1$, поэтому сушествует константа $\lambda$ такая, что

$$
f(2 \cdot)=\lambda f(\cdot) \text { п.в. на } \mathbb{R} \text {. }
$$

Докажем, что (11.27) противоречит $f \in L_{2}(\mathbb{R}) \backslash\{0\}$. Действительно, для любого $C>0$ множества $F_{j}:=\left\{t: 2^{j} \leqslant|t|<2^{j+1}\right.$ и $\left.|f(t)|>C|\lambda|^{j}\right\}$ имеют следуюшие свойства: $F_{j}=2 F_{j-1},\left|F_{j}\right|=2\left|F_{j-1}\right|, j \in \mathbb{Z}$. Если $f \neq 0$, то существует $C>0$ такое, что $\left|F_{0}\right| \neq 0$. Из (11.27) следует, что $|f(t)|>C|\lambda|^{k}$ для $t \in 2^{k} F_{0}$. Поэтому

$$
\int_{\mathbb{R}}|f(t)|^{2} d t \geqslant\left|F_{0}\right| \sum_{k \in \mathbb{Z}}\left(2|\lambda|^{2}\right)^{k},
$$

что противоречит $f \in L_{2}(\mathbb{R})$. 
12. Система Уиттакера-Шеннона-Котельникова. Другим простейшим примером ортогональных всплесков является система Уиттакера-Шеннона-Котельникова (см., например, [19]). Рассмотрим функцию $\varphi^{S}$, имеющую образ Фурье

$$
\widehat{\varphi}^{S}(\omega):=\kappa_{[-\pi, \pi]}(\omega)= \begin{cases}1, & \text { если }|\omega| \leqslant \pi ; \\ 0 & \text { в остальных случаях. }\end{cases}
$$

Ясно, что $\varphi^{S}(t)=\frac{\sin \pi t}{\pi t}$. Очевидно, что $\widehat{\varphi}^{S}$ удовлетворяет условию (11.15), значит, функции $\varphi_{0 k}^{S}(\cdot):=\varphi^{S}(\cdot-k)$ образуют ортонормированный базис в $V_{0}:=\left[\varphi_{0 k}^{S}\right]_{k \in \mathbb{Z}}$. Легко видеть, что $\widehat{V_{0}}:=\left\{\widehat{f}: f \in V_{0}\right\}=L^{2}([-\pi, \pi])$. Кроме того, если $f \in V_{0}$, то

$$
f(t)=\sum_{k \in \mathbb{Z}} f(k) \varphi^{S}(t-k)
$$

Дело в том, что

$$
\left\langle f, \varphi_{0 k}^{S}\right\rangle=\frac{1}{2 \pi}\left\langle\widehat{f}, \widehat{\varphi}_{0 k}^{S}\right\rangle=\frac{1}{2 \pi} \int_{-\pi}^{\pi} \widehat{f}(\omega) e^{i k \omega} d t=f(k)
$$

Если теперь определить для любого целого $j V_{j}:=\left[\varphi_{j k}^{S}\right]_{k \in \mathbb{Z}}$, то легко видеть, что $\left\{V_{j}\right\}_{j \in \mathbb{Z}}$ образуют KМА. Построим соответствуюший всплесковьй базис. Заметим, что $\widehat{\varphi}^{S}(\omega)=m^{S}(\omega / 2) \widehat{\varphi}^{S}(\omega / 2)$, где $2 \pi$-периодическая уточняющая маска $m^{S}$ равна

$$
m^{S}(\omega)=\left\{\begin{array}{l}
1, \text { если }|\omega|<\frac{\pi}{2} ; \\
0, \text { если } \frac{\pi}{2} \leqslant|\omega| \leqslant \pi .
\end{array}\right.
$$

Коэффициенты $\left\{h_{k}\right\}_{k \in \mathbb{Z}}$ из (11.12) можно найти, пользуясь формулой (12.1) (точнее, ее аналогом для функций из $\left.V_{1}\right)$ :

$$
\varphi^{S}(t)=\sum_{k \in \mathbb{Z}} \varphi^{S}(k / 2) \varphi^{S}(2 t-k) .
$$

Значит,

$$
h_{k}^{S}= \begin{cases}1, & \text { если } k=0 ; \\ \frac{\sqrt{2}}{k \pi}(-1)^{(k-1) / 2}, & \text { если } k-\text { нечетное; } \\ 0, & \text { если } k-\text { четное. }\end{cases}
$$

В соответствии с общей схемой всплеск Уиттакера-Шеннона-Котельникова имеет образ Фурье, равный

$$
\begin{aligned}
\widehat{\psi}^{S} & =e^{-i \omega / 2} \overline{m^{S}}(\omega / 2+\pi) \widehat{\varphi}^{S}(\omega / 2) \\
& =e^{-i \omega / 2} \kappa_{[-2 \pi, \pi] \cup[\pi, 2 \pi]}(\omega) \\
& =e^{-i \omega / 2}\left(\widehat{\varphi}^{S}(\omega / 2)-\widehat{\varphi}^{S}(\omega)\right) .
\end{aligned}
$$




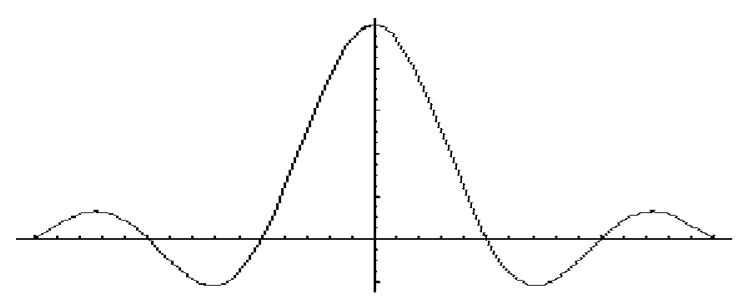

Рис. 10. График $\varphi^{S}(t)$

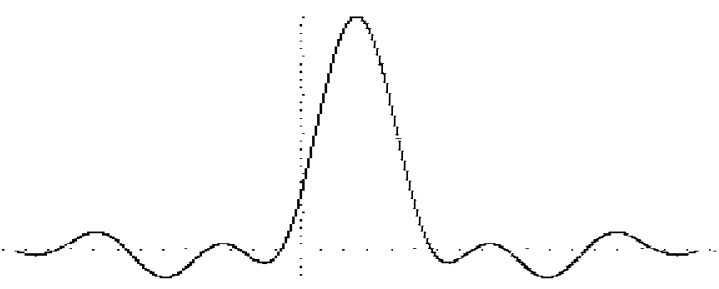

Рис. 11. График $\psi^{S}(t)$

Откуда $\psi^{S}(t)=2 \varphi^{S}(2 t-1)-\varphi^{S}(t-1 / 2)$.

13. Константы неопределенности. Всплески Хаара и Уиттакера-ШеннонаКотельникова представляют собой, условно говоря, два полюса в шкале всплесков. Всплески Хаара имеют прекрасную временную локализованность (компактный носитель), однако плохо локализованы по частоте (преобразование Фурье всплеска Хаара убьвает на бесконечности как $\left.|\omega|^{-1}\right)$. Всплески же Уиттакера-Шеннона-Котельникова, наоборот, имеют компактный спектр (носитель преобразования Фурье), но убывают на бесконечности как $|t|^{-1}$.

Заметим, что $\Delta_{\psi_{j k}}=2^{-j} \Delta_{\psi}, \Delta_{\widehat{\psi}_{j k}}=2^{j} \Delta_{\widehat{\psi}}, j, k \in \mathbb{Z}$. Таким образом, константа неопределенности (см. раздел 5) для всех элементов всплескового базиса одна и та же. Для всплесков Хаара и Уиттакера-Шеннона-Котельникова константа неопределенности равна бесконечности: $\Delta_{\widehat{\psi}^{H}}=\infty, \Delta_{\psi^{S}}=\infty$. Примерами всплесковых базисов с конечной константой неопределенности являются всплески Мейера, Стремберга, Лемари-Бэтла и Добеши.

14. Всплески Мейера. Всплески Мейера [20] являются сглаженным вариантом всплесков Уиттакера-Шеннона-Котельникова. Масштабируюшая функция Мейера $\varphi^{M}$ определяется следуюшим образом. Пусть $\theta(\omega)$ - нечетная бесконечно дифференцируемая функция, равная $\pi / 4$ при $\omega>\pi / 3$ и $-\pi / 4$ при $\omega<-\pi / 3$. Определим четную функцию $\lambda(\omega)$ формулой

$$
\lambda(\omega)= \begin{cases}\frac{\pi}{4}+\theta(\omega-\pi), & \text { если } \omega \in\left[\frac{2 \pi}{3}, \frac{4 \pi}{3}\right] ; \\ \frac{\pi}{4}-\theta\left(\frac{\omega}{2}-\pi\right), & \text { если } \omega \in\left[\frac{4 \pi}{3}, \frac{8 \pi}{3}\right] ; \\ 0, & \text { если } \omega \in\left[0, \frac{2 \pi}{3}\right) \cup\left(\frac{8 \pi}{3}, \infty\right] .\end{cases}
$$


Преобразование Фурье масштабируюшей функции Мейера равно

$$
\widehat{\varphi}^{M}(\omega)= \begin{cases}\cos (\lambda(\omega)), & \text { если }|\omega| \leqslant \frac{4 \pi}{3} \\ 0, & \text { если }|\omega|>\frac{4 \pi}{3} .\end{cases}
$$

Откуда

$$
\varphi^{M}(t)=\frac{1}{2 \pi} \int_{\mathbb{R}} \cos (t \omega) \cos (\lambda(\omega)) d \omega
$$

Легко проверить, что $\widehat{\varphi}^{M}$ удовлетворяет (iii) из теоремы 9.1. Кроме того, $\widehat{\varphi}^{M}(\omega)=$ $m^{M}(\omega / 2) \widehat{\varphi}^{M}(\omega / 2)$, где $2 \pi$-периодическая уточняющая маска $m^{M}(\omega)$ равна

$$
\sum_{l \in \mathbb{Z}} \widehat{\varphi}^{M}(2 \omega+4 l \pi)
$$

Поэтому масштабируюшая функция $\varphi^{M}$ порождает кратномасштабный анализ, и, значит, существует соответствующий всплесковьй базис $\left\{\psi_{j k}^{M}\right\}_{j, k \in \mathbb{Z}}$, где

$$
\widehat{\psi}^{M}(\omega):=e^{-i \omega / 2} \overline{m(\omega / 2+\pi)} \widehat{\varphi}^{M}(\omega / 2)=e^{-i \omega / 2} \sin (\lambda(\omega))
$$

или

$$
\psi^{M}(t)=\frac{1}{2 \pi} \int_{\mathbb{R}} \cos ((t-1 / 2) \omega) \sin (\lambda(\omega)) d \omega
$$

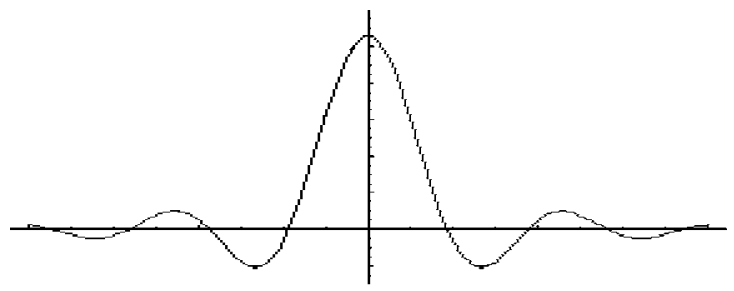

Рис. 12. График $\varphi^{M}(t)$

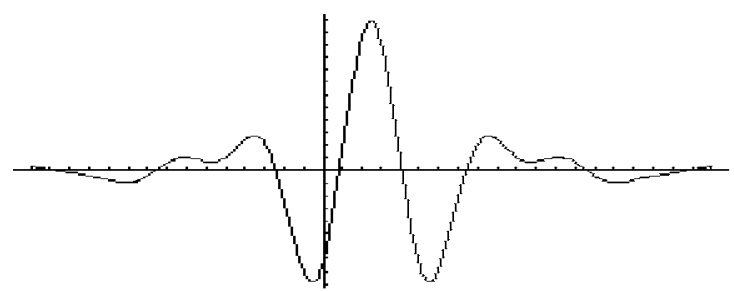

Рис. 13. График $\psi^{M}(t)$ 
Отметим, что масштабирующая функция Мейера, так же как и масштабирующая функция Шеннона (см. (12.2)), удовлетворяет равенству

$$
\varphi^{M}(t)=\sum_{k \in \mathbb{Z}} \varphi^{M}(k / 2) \varphi^{M}(2 t-k)
$$

Действительно,

$$
\begin{aligned}
\left\langle\varphi^{M}, \varphi^{M}(2 \cdot-k)\right\rangle & =\frac{1}{4 \pi} \int_{\mathbb{R}} \widehat{\varphi}^{M}(\omega) \widehat{\varphi}^{M}(\omega / 2) e^{-i k \omega / 2} d \omega \\
& =\frac{1}{4 \pi} \int_{\mathbb{R}} \widehat{\varphi}^{M}(\omega) e^{-i k \omega / 2} d \omega=\frac{1}{2} \varphi^{M}(k / 2) .
\end{aligned}
$$

15. Всплески Лемари-Бэтла и Стремберга. Зафиксируем произвольное натуральное число $m$. Рассмотрим для любого целого $j$ подпространство $V_{j}^{m}$, состоящее из $(m-2)$ раза непрерьвно дифференщируемых функций из $L^{2}(\mathbb{R})$, которые на любом интервале вида $\left[k 2^{-j},(k+1) 2^{-j}\right], k \in \mathbb{Z}$, совпадают с некоторым полиномом степени не вьше $m-1$. Совершенно очевидно, что последовательность $\left\{V_{j}^{m}\right\}_{j \in \mathbb{Z}}$ удовлетворяет свойствам кратномасштабного анализа.

В качестве масштабирующей функции можно взять $B$-сплайн

$$
N^{m}(x):=\left(N^{m-1} * N^{1}\right)(x)=\int_{0}^{1} N^{m-1}(x-t) d t, \quad m \geqslant 2,
$$

где $N^{1}=\kappa_{[0,1)}$. Ясно, что целые сдвиги $N^{m}$ не ортогональны друг другу при $m>1$. Например, при $m=2$

$$
N^{2}(t)= \begin{cases}t, & \text { если } t \in[0,1] ; \\ 2-t, & \text { если } t \in(1,2] ; \\ 0, & \text { если } t \notin[0,2] ;\end{cases}
$$

и функции $N^{2}(\cdot)$ и $N^{2}(\cdot \pm 1)$ не ортогональны друг другу.

Из (15.1) следует, что

$$
\begin{gathered}
\widehat{N^{m}}(\omega)=\left(\frac{1-e^{-i \omega}}{i \omega}\right)^{m}, \quad\left|\widehat{N^{m}}(\omega)\right|=\left|\frac{\sin (\omega / 2)}{\omega / 2}\right|^{m}, \\
\left.\sum_{l \in \mathbb{Z}} \widehat{\mid N^{m}}(\omega+2 l \pi)\right|^{2}=\sum_{l \in \mathbb{Z}}\left|\frac{\sin (\omega / 2+l \pi)}{\omega / 2+l \pi}\right|^{2 m} .
\end{gathered}
$$

Поэтому в силу теоремы $9.2\left\{N^{m}(\cdot-k)\right\}_{k \in \mathbb{Z}}-$ базис Рисса в $V_{0}^{m}$.

Для построения ортогональных сплайн-всплесков определим сначала ортогональную масштабирующую функцию (см. теорему 9.1)

$$
\widehat{\varphi}^{B, m}(\omega)=\widehat{N^{m}}(\omega)\left(\sum_{l \in \mathbb{Z}}\left|\widehat{N}^{m}(\omega+2 l \pi)\right|^{2}\right)^{-1 / 2} .
$$

(Верхний индекс $B$ - первая буква фамилии Ж. Бэтла, всплески которого получатся при таком выборе ортогональной масштабирующей функции.) 
Рассмотрим два случая. Пусть $m$ - четное, $m=2 s, s \in \mathbb{N}$. Тогда

$$
\widehat{N^{m}}(\omega)=\left(\frac{\sin \omega / 2}{\omega / 2}\right)^{2 s} e^{-i \omega s}
$$

Для упрощения выкладок заменим $N^{m}(t)$ на $N^{m}(t+s)$. Тогда

$$
\widehat{N^{m}}(\omega)=\left(\frac{\sin \omega / 2}{\omega / 2}\right)^{2 s}
$$

Для ортогонализации надо посчитать

$$
\left(\left.\sum_{l \in \mathbb{Z}} \widehat{\mid N^{m}}(\omega+2 l \pi)\right|^{2}\right)^{1 / 2}=(\sin \omega / 2)^{2 s}\left(\sum_{l \in \mathbb{Z}}(\omega / 2+l \pi)^{-4 s}\right)^{1 / 2}
$$

Это можно сделать, дифференцируя тождество $\sum_{l \in \mathbb{Z}}(\omega+l \pi)^{-1}=\operatorname{ctg} \omega$. Продифференщировав $(q-1)$ раз, получим

$$
\sum_{l \in \mathbb{Z}} \frac{1}{(\omega+l \pi)^{q}}=\frac{P_{q}(\cos \omega)}{(\sin \omega)^{q}}
$$

где $P_{q}$ - полином степени $q-2$. Для четных $q$ очевидно, что $P_{q}(t)$ строго положителен на $[0,1]$. Окончательно имеем

$$
\widehat{\varphi}^{B, m}(\omega)=\left(\frac{\sin \omega / 2}{\omega / 2}\right)^{m}\left(P_{2 m}(\cos \omega / 2)\right)^{-1 / 2} .
$$

Функция $\varphi^{B, m}$ действительнозначна и симметрична относительно $t=0$.

Если $m$ - нечетно, то аналогичные выкладки показьвают, что

$$
\widehat{\varphi}^{B, m}(\omega)=e^{-i \omega / 2}\left(\frac{\sin \omega / 2}{\omega / 2}\right)^{m}\left(P_{2 m}(\cos \omega / 2)\right)^{-1 / 2}
$$

В этом случае функция $\varphi^{B, m}$ действительнозначна и симметрична относительно $t=1 / 2$.

В соответствии с общей схемой КМА с масштабирующей функцией $\varphi^{B, m}$ позволяет построить всплесковьй базис $\left\{\psi_{j k}^{B, m}\right\}_{j, k \in \mathbb{Z}}$. Этот базис был построен (без использования KMА) в работах: [21], [22]. 


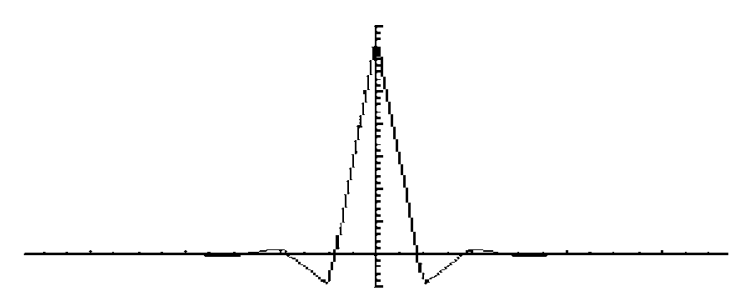

Рис. 14. График $\varphi^{B, 2}(t)$

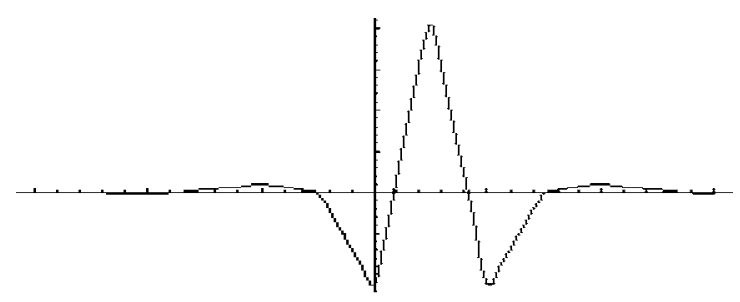

Рис. 15. График $\psi^{B, 2}(t)$

Ортогонализовать $B$-сплайн $N^{m}$ можно чуть-чуть по-другому. Из (15.3) следует, что

$$
\left.\sum_{l \in \mathbb{Z}} \widehat{\mid N^{m}}(\omega+2 l \pi)\right|^{2}=P_{2 m}(\cos \omega / 2) .
$$

Коэффициенты полинома $P_{2 m}(\cos \omega / 2)$ нетрудно найти из следуюших соображений. Очевидно, что

$$
\operatorname{supp} N^{m}=[0, m] .
$$

Остается заметить, что по формуле Планшереля

$$
\begin{aligned}
\left.\frac{1}{2 \pi} \int_{0}^{2 \pi} e^{i k \omega} \sum_{l \in \mathbb{Z}} \widehat{\mid N^{m}}(\omega+2 l \pi)\right|^{2} d \omega & =\frac{1}{2 \pi} \int_{\mathbb{R}} e^{i k \omega}\left|\widehat{N^{m}}(\omega)\right|^{2} d \omega \\
& =\int_{\mathbb{R}} N^{m}(t) N^{m}(t+k) d t .
\end{aligned}
$$

Кроме того, $\left\langle N_{0 k}^{m}, N_{00}^{m}\right\rangle=\left\langle N_{0,-k}^{m}, N_{00}^{m}\right\rangle$.

Напомним хорошо известную лемму Рисса [23, задача 40$]$.

ЛЕмма 15.1. Пусть $A(\xi)=\sum_{-T}^{T} \gamma_{k} e^{i k \xi}-$ тригонометрический полином, положительный или равный нулю на действительной оси. Тогда существует тригонометрический полином $h(\xi)=\sum_{0}^{T} \alpha_{k} e^{i k \xi}$ такой, что $|h(\xi)|^{2}=A(\xi)$. Более того, если коэффициенты $\gamma_{k}$ действительны, то $h(\xi)$ можно тоже выбрать с действительными $\alpha_{k}$.

Из этой леммы следует, что

$$
P_{2 m}(\cos \omega / 2)=a_{m}\left|\left(1+z_{1} e^{i \omega}\right) \cdots\left(1+z_{m-1} e^{i \omega}\right)\right|^{2},
$$


где $\left\{z_{l}\right\}_{l=1}^{m-1}$ всегда можно выбрать внутри единичного круга: $\left|z_{l}\right|<1$. Более того, из результатов И. Шонберга (I. J. Shoenberg) [24] следует, что $\left\{z_{l}\right\}_{l=1}^{m-1}$-положительные числа. Обозначим их $s_{m-1}<\cdots<s_{1}$. Пусть

$$
A_{m}(w):=\sqrt{a_{m}}\left(1+s_{1} e^{i w}\right) \cdots\left(1+s_{m-1} e^{i w}\right)
$$

Тогда функция $\varphi^{S t, m}$, определяемая

$$
\widehat{\varphi^{S t, m}}(\omega)=\frac{\widehat{N}^{m}(\omega)}{A_{m}(\omega)},
$$

так же как и $\varphi^{B, m}$, удовлетворяет условию (iii) из теоремы 9.1 и, следовательно, является ортогональной масштабируюшей для $\left\{V_{j}^{m}\right\}_{j \in \mathbb{Z}}$. Так как $\left(1+s e^{i w}\right)^{-1}=$ $\sum_{l=0}^{\infty}\left(-s e^{i w}\right)^{l}$, равенство $(15.7)$ означает, что $\varphi^{S t, m}$ разлагается в ряд по целым сдвигам влево $B$-сплайна $N^{m}$, причем коэффициенты разложения убывают как геометрическая прогрессия. Значит, $\operatorname{supp} \varphi^{S t, m}=(-\infty, m]$ и $\varphi^{S t, m}(t)$ убьвает экспоненциально при $t \rightarrow-\infty$. КМА с такой масштабирующей функцией порождает всплесковьй базис $\left\{\psi_{j k}^{S t, m}\right\}_{j, k \in \mathbb{Z}}$, построенньй (без использования KMA) в [25].

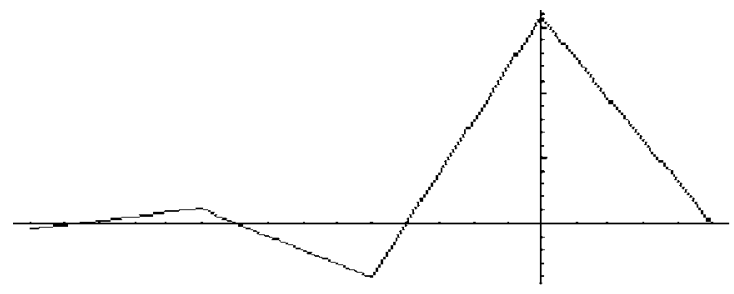

Рис. 16. График $\varphi^{S t, 2}(t)$

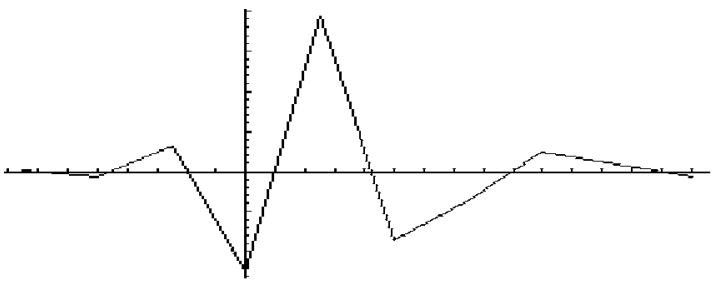

Рис. 17. Графиик $\psi^{S t, 2}(t)$

16. Ортогональные всплески с компактным носителем. Простейший способ построения ортогональных всплесков с компактным носителем основан на использовании ортогональных масштабируюших функций с компактным носителем. В этом случае в последовательности $h_{n}:=\sqrt{2} \int_{\mathbb{R}} \varphi(x) \overline{\varphi(2 x-n)} d x, n \in \mathbb{N}$, из (11.12) только конечное число $h_{n}$ отлично от нуля, и поэтому соответствующий всплеск $\psi($ см. (11.25)) является конечной линейной комбинацией функций с компактньм 
носителем, т.е. сам имеет компактный носитель. Уточняющий фильтр $m_{0}$ будет тригонометрическим полиномом $m_{0}(\omega)=\frac{1}{\sqrt{2}} \sum_{n \in \mathbb{Z}} h_{n} e^{-i n \omega}$. В силу (11.16) $\left|m_{0}(\omega / 2)\right|^{2}+\left|m_{0}(\omega / 2+\pi)\right|^{2} \equiv 1$.

Естественно стараться построить $\varphi$ и $\psi$ достаточно регулярными. Отметим здесь следующее необходимое условие [3].

Теорема 16.1. Пусть $f \in L^{2}(\mathbb{R})$ удовлетворяет условиям

$$
\left\langle f_{j, k}, f_{l, m}\right\rangle=\delta_{j, l} \delta_{k, m}
$$

где $f_{j, k}(x)=2^{j / 2} f\left(2^{j} x-k\right)$. Предположим, что $f$ имеет компактный носитель, $f \in C^{m}(\mathbb{R})$ и $f^{(l)}$ ограничень при $l \leqslant m$. Тогда

$$
\int_{\mathbb{R}} x^{l} f(x) d x=0 \quad \text { для } \quad l=0,1, \ldots, m .
$$

Свойство (16.1) эквивалентно тому, что $\widehat{\psi}^{(l)}(0)=0$ при $l=0,1, \ldots, m$. Так как $\widehat{\psi}(\omega)=e^{-i \omega / 2} \overline{m_{0}(\omega / 2+\pi)} \widehat{\varphi}(\omega / 2)$ и $\widehat{\varphi}(0)=1$, то из $\psi \in C^{m}(\mathbb{R})$ следует, что $m_{0}$ имеет нуль кратности $m+1$ в $\pi$ или $m_{0}(\omega)=\left(\frac{1+e^{i \omega}}{2}\right)^{m+1} L(\omega)$, где $L$ - некоторый тригонометрический полином.

Итак, будем искать решения (11.16) в виде $m_{0}(\omega)=\left(\frac{1+e^{i \omega}}{2}\right)^{N} L(\omega)$. Заметим, что

$$
\left|m_{0}(\omega)\right|^{2}=\left(\cos ^{2} \frac{\omega}{2}\right)^{N}|L(\omega)|^{2}=\left(\cos ^{2} \frac{\omega}{2}\right)^{N} P\left(\sin ^{2} \frac{\omega}{2}\right)
$$

где $P\left(\sin ^{2} \frac{\omega}{2}\right):=|L(\omega)|^{2}$. Подставляя это выражение в (11.16), получаем уравнение на $P$ :

$$
x^{N} P(1-x)+(1-x)^{N} P(x)=1 .
$$

Так как $x^{N}$ и $(1-x)^{N}$ - взаимно-простые полиномы степени $N$, то по теореме Безу сушествует единственньй полином $P_{N-1}$ степени $N-1$, удовлетворяюший (16.2):

$$
P_{N-1}(x)=\sum_{k=0}^{N-1}\left(\begin{array}{c}
N-1+k \\
k
\end{array}\right) x^{k}
$$

Очевидно, что $P_{N-1}(x)>0$. Сушествуют решения (16.2) более высокой степени $P(x)=P_{N-1}(x)+x^{N} R\left(x-\frac{1}{2}\right)$, где $R$ - произвольньй нечетньй полином. Для простоты рассмотрим здесь только случай $R=0$.

Зная $P$, полином $m_{0}$ находится при помоши леммы 15.1 .

Итак, пусть $N \in \mathbb{N}$. Фильтрами Добеши называют тригонометрические полиномы

$$
d_{N}(\omega)=2^{-1 / 2} \sum_{l=0}^{2 N-1} h_{N}(l) e^{i l \omega}, \quad h_{N}(l) \in \mathbb{R}
$$

удовлетворяющие равенствам

$$
\left|d_{N}(\omega)\right|^{2}=\left(\cos ^{2} \frac{\omega}{2}\right)^{N} P_{N-1}\left(\sin ^{2} \frac{\omega}{2}\right) .
$$

В [26] доказана следующая 
ТЕОрема 16.2. Функиия $\varphi^{D, N}$, определенная в образах Фурье как

$$
\widehat{\varphi^{D, N}}(\omega):=\prod_{l=1}^{\infty} d_{N}\left(\omega 2^{-l}\right),
$$

является ортогональной масштабирующей функцией. Соответствующий всплеск $\psi^{D, N}$, определяемый формулой

$$
\widehat{\psi^{D, N}}(\omega)=e^{-i \omega / 2} \overline{d_{N}(\omega / 2+\pi)} \widehat{\varphi^{D, N}}(\omega / 2),
$$

порождает ортонормированный базис в $L^{2}(\mathbb{R})$ :

$$
\left\{\psi_{j k}^{D, N}(\cdot):=2^{j / 2} \psi^{D, N}\left(2^{j} \cdot-k\right)\right\}_{j \in \mathbb{Z}, k \in \mathbb{Z}}
$$

Более того, $\operatorname{supp} \psi^{D, N}=[-(N-1), N]$, и существует $\lambda>0$ такая, что $\psi^{D, N} \in$ $C^{\lambda N}$, где $C^{\alpha}:=\left\{f: \int_{\mathbb{R}} \hat{f}(\omega)(1+|\omega|)^{\alpha} d \omega<\infty\right\}, \alpha>0$.

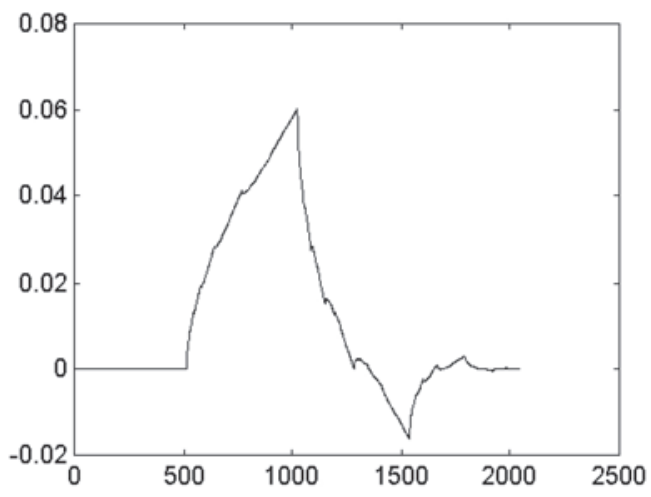

Рис. 18. Графики $\varphi^{D, 2}(t)$

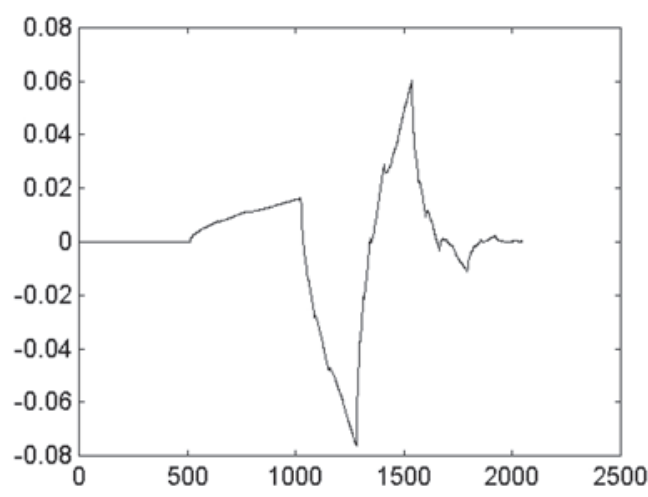

Рис. 19. График $\psi^{D, 2}(t)$ 


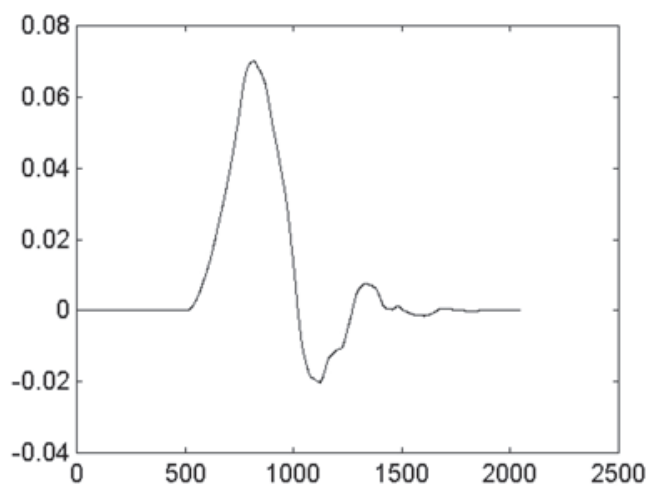

Рис. 20. График $\varphi^{D, 4}(t)$

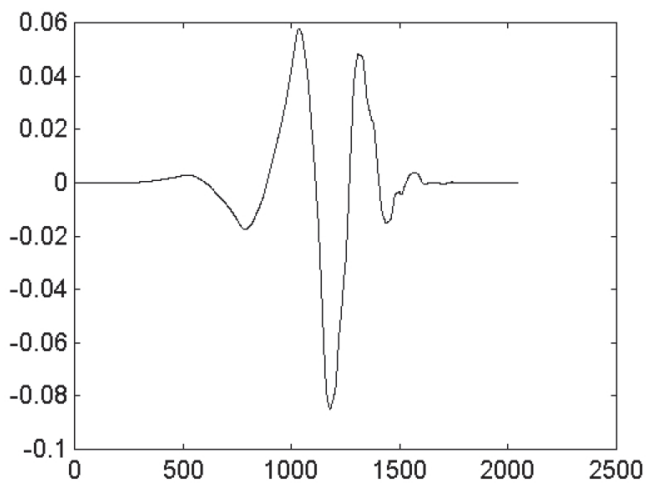

Рис. 21. График $\psi^{D, 4}(t)$

17. Быстрые алгоритмы. Кратномасштабньй анализ позволяет быстро вычислять всплесковые коэффициенты заданной функции. Предположим, что нам известны скалярные произведения $f$ с $\left\{\varphi_{j k}\right\}_{k \in \mathbb{Z}}$ для некоторого $j$. Не ограничивая обшности, можно считать $j=0$ (к этому случаю всегда можно перейти соответствуюшей заменой переменных). Зная $\left\langle f, \varphi_{0 k}\right\rangle, k \in \mathbb{Z}$, легко подсчитать $\left\langle f, \psi_{j k}\right\rangle$ для $j<0$. Действительно, $\psi=\sum_{k \in \mathbb{Z}} g_{k} \varphi_{1 k}$, где $g_{k}=(-1)^{k} h_{1-k}$ (см. (11.25)). Следовательно,

$$
\begin{aligned}
\psi_{j k}(t) & =2^{j / 2} \psi\left(2^{j} t-k\right)=2^{j / 2} \sum_{l \in \mathbb{Z}} \varphi\left(2^{j} t-2 k-l\right) \\
& =\sum_{l \in \mathbb{Z}} g_{l} \varphi_{j+1,2 k+l}(t)=\sum_{l \in \mathbb{Z}} g_{l-2 k} \varphi_{j+1, l}(t) .
\end{aligned}
$$

Значит,

$$
\left\langle f, \psi_{-1, k}\right\rangle=\sum_{l \in \mathbb{Z}} \overline{g_{l-2 k}}\left\langle f, \varphi_{0 l}\right\rangle,
$$

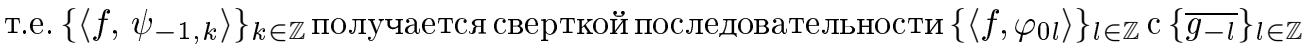
с последующим выбором только четных элементов. Аналогично вьполняется переход 
от слоя $j$ к $j-1$ :

$$
\left\langle f, \psi_{j-1, k}\right\rangle=\sum_{l \in \mathbb{Z}} \overline{g_{l-2 k}}\left\langle f, \varphi_{j, l}\right\rangle
$$

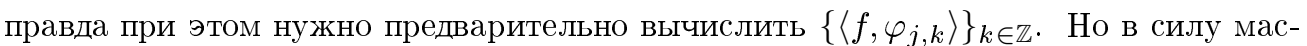
штабного равенства

$$
\varphi_{j, k}=\sum_{l \in \mathbb{Z}} h_{l-2 k} \varphi_{j+1, l}
$$

Поэтому

$$
\left\langle f, \varphi_{j, k}\right\rangle=\sum_{l \in \mathbb{Z}} \overline{h_{l-2 k}}\left\langle f, \varphi_{j+1, l}\right\rangle
$$

Итак, начиная с $\left\{\left\langle f, \varphi_{0, k}\right\rangle\right\}_{k \in \mathbb{Z}}$, можно вычислить $\left\{\left\langle f, \psi_{-1, k}\right\rangle\right\}_{k \in \mathbb{Z}}$ по (17.2) и $\{\langle f, \varphi-1, k\rangle\}_{k \in \mathbb{Z}}$ по (17.4). Затем мы можем применить (17.2) и (17.4) снова и

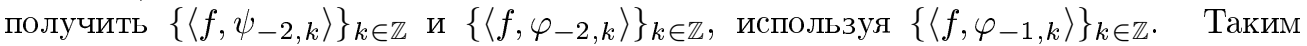
образом, на каждом шаге вычисляются не только всплесковые коэффищиенты $j$-го

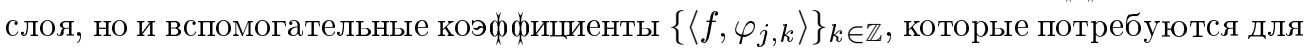
нахождения всплесковых коэффициентов в $(j-1)$-м слое.

В целом весь процесс можно рассматривать как последовательное вычисление более грубых приближений функции $f$ вместе с фиксацией деталей, необходимых для получения более точного приближения из более грубого. С этой точки зрения мы начинаем с приближения $f^{0}=P_{0} f$ (напоминаем, что $P_{j}$ - это ортогональньй проектор на $V_{j}$; через $Q_{j}$ мы будем обозначать проектор на $W_{j}$ ). Далее мы разлагаем $f^{0} \in V_{0}=V_{-1} \oplus W_{-1}$ на $f^{-1}$ и $\delta^{-1}: f^{0}=f^{-1}+\delta^{-1}$, где $f^{-1}=P_{-1} f^{0}=P_{-1} f-$ более грубое приближение $f$ в шкале кратномасштабного анализа, а $\delta^{-1}=f^{0}-f^{-1}=$ $Q_{-1} f^{0}=Q_{-1} f-$ это "потеря" в информации при отображении $f^{0} \rightarrow f^{-1}$. В каждом из пространств $V_{j}$ и $W_{j}$ есть ортонормированньй базис $\left\{\varphi_{j, k}\right\}_{k \in \mathbb{Z}}$ и $\left\{\psi_{j, k}\right\}_{k \in \mathbb{Z}}$, поэтому

$$
f^{0}=\sum_{k \in \mathbb{Z}} s_{k}^{0} \varphi_{0 k}, \quad f^{-1}=\sum_{k \in \mathbb{Z}} s_{k}^{-1} \varphi_{-1, k}, \quad \delta^{-1}=\sum_{k \in \mathbb{Z}} d_{k}^{-1} \psi_{-1, k} .
$$

Таким образом, формулы $(17.2),(17.4)$ задают преобразование коэффициентов при переходе от ортонормированного базиса $\left\{\varphi_{0 k}\right\}_{k \in \mathbb{Z}}$ в $V_{0}$ к ортонормированному базису $\left\{\varphi_{-1, k}\right\}_{k \in \mathbb{Z}} \cup\left\{\psi_{-1, k}\right\}_{k \in \mathbb{Z}}$ :

$$
s_{k}^{-1}=\sum_{l \in \mathbb{Z}} \overline{h_{l-2 k}} s_{k}^{0}, \quad d_{k}^{-1}=\sum_{l \in \mathbb{Z}} \overline{g_{l-2 k}} s_{k}^{0} .
$$

Если обозначить $a:=\left\{a_{l}\right\}_{l \in \mathbb{Z}}, A:=\left\{a_{-l}\right\}_{l \in \mathbb{Z}}$ и $(A b)_{k}=\sum_{l \in \mathbb{Z}} A_{2 k-l} b_{l}$, то (17.5) можно записать в виде $s^{-1}=\bar{H} s^{0}, d^{-1}=\bar{G} s^{0}$.

Приближение $f^{-1} \in V_{-1}=V_{-2} \oplus W_{-2}$ может быть снова разложено:

$$
\begin{aligned}
& f^{-1}=f^{-2}+\delta^{-2}, \quad f^{-2} \in V_{-2}, \quad \delta^{-2} \in W_{-2}, \\
& f^{-2}=\sum_{k \in \mathbb{Z}} s_{k}^{-2} \varphi_{-2, k}, \quad \delta^{-2}=\sum_{k \in \mathbb{Z}} d_{k}^{-2} \psi_{-2, k} .
\end{aligned}
$$


Опять $s^{-2}=\bar{H} s^{-1}, d^{-2}=\bar{G} s^{-1}$. Схематично весь процесс можно изобразить так:

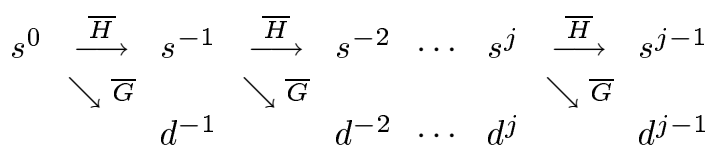

На практике, после выполнения конечного числа шагов процесс прекращается, что означает, что исходная информация $\left\{\left\langle f, \varphi_{0 k}\right\rangle\right\}_{k \in \mathbb{Z}}=s^{0}$ преобразована в

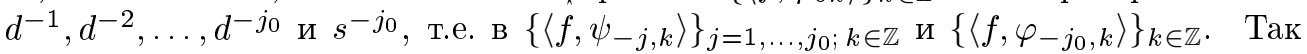
как преобразования вьполнялись при помощи изменения ортогональных базисов, обратная операция задается сопряженной матрицей. Точнее:

$$
f^{j+1}=f^{j}+\delta^{j}=\sum_{k \in \mathbb{Z}} s_{k}^{j} \varphi_{j k}+\sum_{k \in \mathbb{Z}} d_{k}^{j} \psi_{j k} .
$$

Следовательно (см. (17.1), (17.3)),

$$
\begin{aligned}
s_{k}^{j+1} & =\left\langle f^{j+1}, \varphi_{j+1, k}\right\rangle \\
& =\sum_{l \in \mathbb{Z}} s_{l}^{j}\left\langle\varphi_{j l}, \varphi_{j+1, k}\right\rangle+\sum_{l \in \mathbb{Z}} d_{l}^{j}\left\langle\psi_{j l}, \psi_{j+1, k}\right\rangle \\
& =\sum_{l \in \mathbb{Z}} h_{k-2 l} s_{l}^{j}+\sum_{l \in \mathbb{Z}} g_{k-2 l} d_{l}^{j} .
\end{aligned}
$$

Если обозначить $(\widetilde{a} b)_{k}:=\sum_{l \in \mathbb{Z}} a_{k-2 l} b_{l}$, то $s^{j+1}=\widetilde{h} s^{j}+\widetilde{g} d^{j}$ и схематично процесс восстановления вьглядит так:

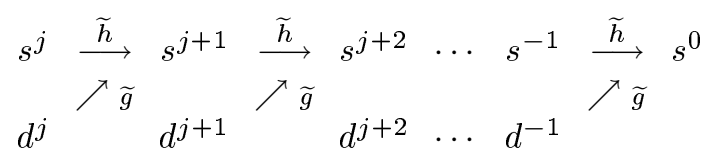

Важнейшей чертой изложенного алгоритма разложения и восстановления является его быстрота. Например, для системы Хаара имеем следующее. Пусть исходная информация состояла из $2^{N}$ чисел $\left\{s_{k}^{0}\right\}_{k=0}^{2^{N}-1}$. Тогда на первом шаге вычисляется $2^{N-1}$ вспомогательных чисел $\left\{s_{k}^{-1}\right\}_{k=0}^{2^{N-1}-1}: s_{k}^{-1}=\left(s_{2 k}^{0}+s_{2 k+1}^{0}\right) / \sqrt{2}$ и $2^{N-1}$ коэффищиентов $\left\{d_{k}^{-1}\right\}_{k=0}^{2^{N-1}-1}: d_{k}^{-1}=\left(s_{2 k}^{0}-s_{2 k+1}^{0}\right) \sqrt{2}$. На каждом следуюшем шаге количество вспомогательных чисел и коэффициентов уменьшается в два раза. Количество операций во всем алгоритме разложения равно $2 \cdot 2^{N}\left(\frac{1}{2}+\frac{1}{4}+\cdots\right)=2 \cdot 2^{N}$. Для более сложных всплесковых базисов вычисления вспомогательных чисел (условно говоря, “средних") и коэффищиентов ("разностей”) требуют более чем два предыдуших числа, но рассуждения о количестве коэффициентов на каждом слое остаются в силе. Если "обобшенные средние" и "разности" используют $K$ предыдуших чисел, то обшее число операций равно $2 K N$ ( $K N$ - умножений, $K N$ - сложений).

Следует отметить, что быстрьй алгоритм разложения и восстановления по всплесковому базису (коротко, быстрое всплесковое преобразование (БВП)) был известен в цифровой обработке сигналов под названием полосовая фильтрация с точным восстановлением (subband filtering scheme with exact reconstruction). Эта схема была предложена до появления теории всплесков в работах: [27]-[29]. 
18. Полуортогональные сплайн-всплески с компактным носителем.

Пусть $\varphi$ - масштабируюшая функция с компактным носителем, порождающая КМА $\left\{V_{j}\right\}_{j \in \mathbb{Z}}$ в $L^{2}(\mathbb{R})$. В этом случае можно построить соответствуюший полуортогональньй всплеск $\psi$ с компактньп носителем. В этом разделе мы не предполагаем ортонормированность последовательности $\{\varphi(\cdot-k)\}_{k \in \mathbb{Z}}$ (дело в том, что ортогонализация (9.2) масштабируюшей функции с компактным носителем приводит к потере компактного носителя).

Пусть $\operatorname{supp} \varphi=[0, M], M \in \mathbb{N}$. Тогда $\varphi=\sum_{-M+1}^{2 M-1} h_{k} \varphi_{1 k}$. Для удобства будем рассматривать действительнозначные $\varphi$. Очевидно, что автокоррелящионная функция $\Phi(t):=\int_{\mathbb{R}} \varphi(x-t) \varphi(x) d x$ является четной функцией и $\operatorname{supp} \Phi=[-M, M]$. Пусть $n_{\varphi}$ - наибольшее целое, для которого $\Phi\left(n_{\varphi}\right) \neq 0$. Так как $\Phi$ - непрерьвная функция, то $\Phi(M)=0$ и $0 \leqslant n_{\varphi} \leqslant M-1$. Аналогично (15.6) получаем, что

$$
\sum_{l \in \mathbb{Z}}|\widehat{\varphi}(\omega+2 l \pi)|^{2}=E_{\varphi}\left(e^{-i \omega}\right),
$$

где $E_{\varphi}(z):=\sum_{k=-n_{\varphi}}^{n_{\varphi}} \Phi(k) z^{k}$. Полином $P_{\varphi}(z)$ назьвают полиномом Эйлера- $\Phi$ робениуса для масштабируюшей функции $\varphi$.

Построение полуортогонального всплескового базиса аналогично построению ортогонального (см. раздел 11). Прежде всего охарактеризуем функции $\psi \in W_{0}$, где $V_{0} \oplus W_{0}=V_{1}, W_{0} \perp V_{0}$. Так как $W_{0} \subset V_{1}$, то $\psi=\sum_{k} r_{k} \varphi_{1 k}$. В образах Фурье имеем

$$
\widehat{\psi}(\omega)=R\left(e^{-i \omega / 2}\right) \widehat{\varphi}(\omega / 2),
$$

где $R(z)=\frac{1}{\sqrt{2}} \sum_{k \in \mathbb{Z}} r_{k} z^{k}-2 \pi$-периодическая функция из $L^{2}[0,2 \pi]$. Аналогично для $\varphi$ :

$$
\varphi=\sum_{k} p_{k} \varphi_{1 k}, \quad \widehat{\varphi}(\omega)=P\left(e^{-i \omega / 2}\right) \widehat{\varphi}(\omega / 2), \quad P(z)=\frac{1}{\sqrt{2}} \sum_{k \in \mathbb{Z}} p_{k} z^{k}
$$

Пусть $z=e^{-i \omega / 2}$. Применяя тождество Планшереля, имеем

$$
\begin{aligned}
\langle\varphi(\cdot-l), \psi(\cdot)\rangle & =\frac{1}{2 \pi} \int_{\mathbb{R}} \widehat{\varphi}(\omega) e^{-i l \omega} \overline{\widehat{\psi}(\omega)} d \omega \\
& =\frac{1}{2 \pi} \int_{\mathbb{R}}|\widehat{\varphi}(\omega / 2)|^{2} P(z) \overline{R(z)} e^{-i l \omega} d \omega \\
& =\frac{1}{2 \pi} \int_{0}^{4 \pi}\left\{\sum_{k \in \mathbb{Z}}|\widehat{\varphi}(\omega / 2+2 \pi k)|^{2}\right\} P(z) \overline{R(z)} e^{-i l \omega} d \omega \\
& =\frac{1}{2 \pi} \int_{0}^{4 \pi} E_{\varphi}(z) P(z) \overline{R(z)} e^{-i l \omega} d \omega \\
& =\frac{1}{2 \pi} \int_{0}^{2 \pi}\left\{E_{\varphi}(z) P(z) \overline{R(z)}+E_{\varphi}(-z) P(-z) \overline{R(-z)}\right\} e^{-i l \omega} d \omega .
\end{aligned}
$$

Откуда следует, что $\psi \in W_{0}$ тогда и только тогда, когда

$$
E_{\varphi}(z) P(z) \overline{R(z)}+E_{\varphi}(-z) P(-z) \overline{R(-z)}=0, \quad|z|=1 .
$$


Если предположить, что коэффициенты $R$ действительны, то нетрудно указать общее решение (18.1):

$$
R(z)=z K\left(z^{2}\right) P\left(-\frac{1}{z}\right) E_{\varphi}(-z), \quad|z|=1,
$$

где $K(z)$ - произвольная квадратично-суммируемая функция на единичной окружности $|z|=1$. Здесь использовано то, что $E_{\varphi}\left(z^{-1}\right)=E_{\varphi}(z)$ при $|z|=1$, так как $\Phi(-k)=\Phi(k)$.

Если $\varphi$ имеет компактный носитель, то $P$ и $E_{\varphi}$ являются полиномами Лорана. Из (18.2) следует, что $R$ будет тоже полиномом Лорана, если $K$ - полином Лорана.

Теорема 18.1. Полуортогональный всплеск с минимальным компактным носителем, соответствующий, , определяется двухмасштабным соотношением

$$
\psi:=\sum_{k} q_{k} \varphi_{1, k}
$$

или, в образах Фурье,

$$
\widehat{\psi}(\omega):=Q\left(e^{-i \omega / 2}\right) \widehat{\varphi}(\omega / 2),
$$

где $Q(z):=\frac{1}{\sqrt{2}} q_{k} z^{k}=c z^{2 N+1} P(-1 / z) E_{\varphi}(-z)$. Здесь $N-$ произвольное челое, $c$ - произвольная ненулевая константа. В частности, если положить $N$ равным иелой части $\left(M+n_{\varphi}\right) / 2$, то $Q$ будет алгебраическим полиномом и $\psi:=$ $\sum_{k=0}^{M+2 n_{\varphi}} q_{k} \varphi_{1, k}$

Последовательность $\{\psi(\cdot-k)\}_{k \in \mathbb{Z}}$ образует базис Рисса в $W_{0}$, a $\left\{\psi_{j, k}(t):=\right.$ $\left.2^{j / 2} \psi(2 t-k)\right\}_{j, k \in \mathbb{Z}}$ - полуортогональный базис в $L^{2}(\mathbb{R})$.

Применим эту теорему к $B$-сплайну $\varphi=N^{m}, m \in \mathbb{N}$. В этом случае $M=m$, $n_{\varphi}=m-1$. Полагая $N=m-1, c=-1$, имеем

$$
\begin{aligned}
Q_{m}(z) & =-z^{2 m-1} P\left(-\frac{1}{z}\right) E(-z) \\
& =-z^{2 m-1}\left(\frac{z-1}{2 z}\right)^{m} \sum_{k=-m+1}^{m-1}(-1)^{k} N^{2 m}(m+k) z^{k} \\
& =\left(\frac{1-z}{2}\right)^{m} \sum_{k=0}^{2 m-2}(-1)^{k} N^{2 m}(k+1) z^{k}=\frac{1}{\sqrt{2}} \sum_{k=0}^{3 m-2} q_{m, k} z^{k},
\end{aligned}
$$

где

$$
q_{m, k}=\frac{(-1)^{k}}{2^{m-\frac{1}{2}}} \sum_{l=0}^{m} N^{2 m}(k-l+1), \quad k=0, \ldots, 3 m-2 .
$$

Здесь использованы легко проверяемые соотношения:

$$
N^{m}(t)=\sum_{k=0}^{m} 2^{-m+1}\left(\begin{array}{c}
m \\
k
\end{array}\right) N^{m}(2 t-k), \quad E_{N^{m}}(z)=\sum_{-m+1}^{m-1} N^{2 m}(m+k) z^{k} .
$$




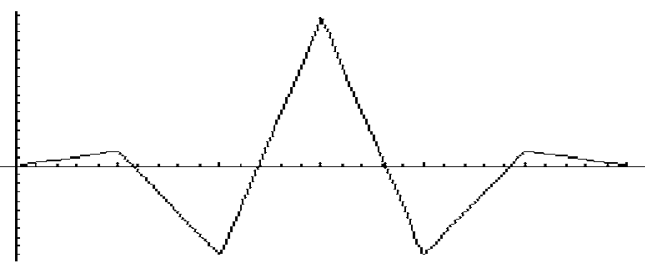

Рис. 22. График $\psi^{2}(t)$

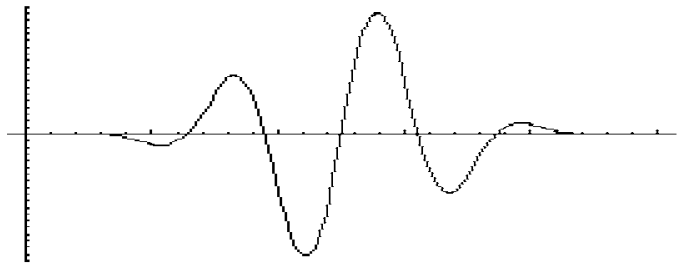

Рис. 23. График $\psi^{3}(t)$

Окончательно, полуортогональный сплайн-всплеск порядка $m$ равен

$$
\psi^{m}(t)=\sum_{k=0}^{3 m-2} \sqrt{2} q_{m, k} N^{m}(2 t-k), \quad \operatorname{supp} \psi^{m}=[0,2 m-1] .
$$

19. Регулярные KMA в $L^{2}\left(\mathbb{R}^{n}\right)$. Перейдем от рассмотрения одномерных KMA к многомерным. Следующее определение обобщает определение 11.1.

ОПРЕДЕлЕНИЕ 19.1. Кратномасштабньй анализ (KMA) - это последовательность $\left\{V_{j}\right\}_{j \in \mathbb{Z}}$ замкнутых подпространств $L^{2}\left(\mathbb{R}^{n}\right)$, удовлетворяющая следуюшим свойствам:

$$
\begin{gathered}
V_{j \in \mathbb{Z}} \subset V_{j+1} ; \\
\bigcap_{j \in \mathbb{Z}} V_{j}=\{0\} ; \\
f \in V_{j} \Leftrightarrow f\left(2^{-j} \cdot\right) \in V_{0} ; \\
f \in V_{0} \Leftrightarrow f(\cdot-k) \in V_{0} \text { для любого } k \in \mathbb{Z}^{n} ;
\end{gathered}
$$

существует функция $g \in V_{0}$ такая, что последовательность

$$
\{g(\cdot-k)\}_{k \in \mathbb{Z}^{n}} \text { образует базис Рисса в } V_{0} \text {. }
$$

Напомним, что последовательность $\left\{g_{0 k}(\cdot):=g(\cdot-k)\right\}_{k \in \mathbb{Z}^{n}}$ является базисом Рисса в $V_{0}$, если $\left[g_{0 k}\right]_{k \in \mathbb{Z}^{n}}=V_{0}$ и сушествуют две константы $A>0, B>0$ такие, что

$$
A\left(\sum_{k \in \mathbb{Z}^{n}}\left|c_{k}\right|^{2}\right)^{1 / 2} \leqslant\left\|\sum_{k \in \mathbb{Z}} c_{k} g_{0 k}\right\|_{L^{2}(\mathbb{R})} \leqslant B\left(\sum_{k \in \mathbb{Z}^{n}}\left|c_{k}\right|^{2}\right)^{1 / 2}
$$


для любой последовательности чисел $\left\{c_{k}\right\}_{k \in \mathbb{Z}^{n}}$.

Эффективность использования всплесковых базисов в функциональных пространствах, отличных от $L^{2}\left(\mathbb{R}^{n}\right)$, зависит от регулярности соответствующего KMA.

ОПРеДЕЛЕНИЕ 19.2. КМА $\left\{V_{j}\right\}_{j \in \mathbb{Z}^{n}}$ называется $r$-регулярньм $(r \in \mathbb{N})$, если функция $g(x)$ в (19.6) может быть выбрана так, что

$$
\left|\partial^{\alpha} g(x)\right| \leqslant C_{m}(1+|x|)^{-m}
$$

для любого целого $m \in \mathbb{N}$ и для любого мультииндекса $\alpha=\left(\alpha_{1}, \ldots, \alpha_{n}\right)$, удовлетворяющего $|\alpha| \leqslant r$.

Следуюшая теорема показывает, как перейти от базиса Рисса к каноническому ортонормированному базису (см. для сравнения одномерньй случай в теоремах 9.1-9.3).

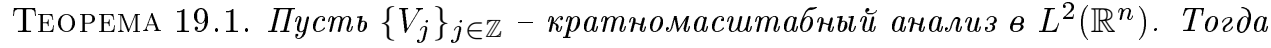
существуют две константы $c_{2} \geqslant c_{1}>0$ такие, что для почти всех $\omega \in \mathbb{R}^{n}$ имеем

$$
c_{1} \leqslant\left(\sum_{k \in \mathbb{Z}^{n}}|\widehat{g}(\omega+2 k \pi)|^{2}\right)^{1 / 2} \leqslant c_{2} .
$$

Далее, если $\varphi \in L^{2}\left(\mathbb{R}^{n}\right)$ определена в образах Фурье

$$
\widehat{\varphi}(\omega)=\widehat{g}(\omega)\left(\sum_{k \in \mathbb{Z}^{n}}|\widehat{g}(\omega+2 k \pi)|^{2}\right)^{-1 / 2},
$$

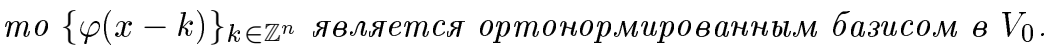

Наконеи, пусть последовательность $\{f(x-k)\}_{k \in \mathbb{Z}^{n}}$ ортонормирована, где функция $f \in V_{0}$. Тогда эта последовательность является ортонормированныц базисом в $V_{0}$ и $\widehat{f}(\omega)=\theta(\omega) \widehat{\varphi}(\omega)$, где $\theta(\omega) \in C^{\infty}\left(\mathbb{R}^{n}\right),|\theta(\omega)|=1$ почти всюду и $\theta(\omega+2 k \pi)=\theta(\omega)$ для любого $k \in \mathbb{Z}^{n}$.

Следуюшая теорема показывает, что ортогонализация (19.9) сохраняет регулярность КМА.

ТеОРема 19.2. Пусть $\left\{V_{j}\right\}_{j \in \mathbb{Z}}-r$-регулярный кратномаситабный анализ в $L^{2}\left(\mathbb{R}^{n}\right)$. Тогда функция $\varphi \in V_{0}$, определенная в (19.9), удовлетворяет оченке

$$
\left|\partial^{\alpha} \varphi(x)\right| \leqslant C_{m}(1+|x|)^{-m}
$$

для любого челого $m \in \mathbb{N}$ и для любого мультииндекса $\alpha \in \mathbb{N}^{n}$, удовлетворяющего $|\alpha| \leqslant r$.

Покажем, как получить КМА в $L^{2}\left(\mathbb{R}^{2}\right)$, используя одномерный KMA $\left\{\mathscr{V}_{j}\right\}_{j \in \mathbb{Z}}$ в $L^{2}(\mathbb{R})$. Определим $V_{j} \subset L^{2}\left(\mathbb{R}^{2}\right)$ как замыкание в $L^{2}\left(\mathbb{R}^{2}\right)$-норме алгебраического тензорного произведения $\mathscr{V}_{j} \otimes \mathscr{V}_{j}$. Полученньй KMA назьвают сепарабельным. Если многомерньй KMA нельзя получить описанньм способом, то его назьвают несепарабельным. 
В сепарабельном случае ортонормированньй базис в $V_{0}$ состоит из произведений $\varphi(x-k) \varphi(y-k),(k, l) \in \mathbb{Z}^{2}$. Другими словами, полагая $\varphi(x, y):=\varphi(x) \varphi(y)$, получаем, что ортонормированный базис в $V_{0}$ является орбитой функции $\varphi$ под действием $\mathbb{Z}^{2}$.

Пусть $\mathscr{W}_{0}$ - ортогональное дополнение $\mathscr{V}_{0}$ до $\mathscr{V}_{1}$. Тогда

$$
V_{1}=V_{0} \oplus \overline{\mathscr{V}_{0} \otimes \mathscr{W}_{0}} \oplus \overline{\mathscr{W}_{0} \otimes \mathscr{V}_{0}} \oplus \overline{\mathscr{W}_{0} \otimes \mathscr{W}_{0}}
$$

Действительно, $V_{1}=\overline{\left(\mathscr{V}_{0} \oplus \mathscr{W}_{0}\right) \otimes\left(\mathscr{V}_{0} \oplus \mathscr{W}_{0}\right)}$ и достаточно воспользоваться дистрибутивностью тензорного произведение по отношению к сложению. Пусть $W_{0}$ обозначает ортогональное дополнение $V_{0}$ до $V_{1}$. Тогда $W_{0}=W_{0,1} \oplus W_{1,0} \oplus W_{1,1}$, где $W_{0,1}=\overline{\mathscr{V}_{0} \otimes \mathscr{W}_{0}}, W_{1,0}=\overline{\mathscr{W}_{0} \otimes \mathscr{V}_{0}}, W_{1,1}=\overline{\mathscr{W}_{0} \otimes \mathscr{W}_{0}}$.

Для получения ортонормированного базиса в $W_{0}$ надо взять объединение последовательностей $\varphi(x-k) \psi(y-l), \psi(x-k) \varphi(y-l)$ и $\psi(x-k) \psi(y-l), k, l \in \mathbb{Z}^{2}$, которые являются ортонормированньми базисами в $W_{0,1}, W_{1,0}$ и $W_{1,1}$.

Проблема построения всплескового базиса на основе $\mathrm{KMA} \mathrm{в} L^{2}\left(\mathbb{R}^{n}\right)$ в общем случае является более сложной. Однако в [30] доказана

Теорема 19.3. Пусть $\left\{V_{j}\right\}_{j \in \mathbb{Z}}-r$-регулярный кратномасштабный анализ в $L^{2}\left(R^{n}\right)$. $W_{j}$ - ортогональное дополнение $V_{j}$ до $V_{j+1}$. Тогда существует $q:=2^{n}-1$ функций $\psi_{1}, \ldots, \psi_{q}$ из $V_{1}$ со следующими свойствами:

$$
\left|\partial^{\alpha} \psi_{l}(x)\right| \leqslant C_{N}(1+|x|)^{-N}
$$

для любого мультииндекса $\alpha \in \mathbb{N}^{n} c|\alpha| \leqslant r$, любого $x \in \mathbb{R}^{n}$ и любого $N \geqslant 1$

$\left\{\psi_{l}(x-k), 1 \leqslant l \leqslant q, k \in \mathbb{Z}^{n}\right\}$ является ортонормированным базисом в $W_{0}$.

СлЕДСТВИЕ 19.1. Функции $2^{n j / 2} \psi_{l}\left(2^{j} x-k\right), 1 \leqslant l \leqslant q, k \in \mathbb{Z}^{n}, j \in \mathbb{Z}$, образуют ортонормированный базис в $L^{2}\left(\mathbb{R}^{n}\right)$.

ЗАмЕчАНИЕ 19.1. Не известен метод построения несепарабельного многомерного KMA $\left\{V_{j}\right\}_{j \in \mathbb{Z}}$ в $L^{2}\left(\mathbb{R}^{n}\right)$, у которого всплесковьй базис состоит из компактных функций. Дело в том, что алгоритм К. Грошенига (K. Gröchenig) даже при применении к компактной масштабирующей функции $\varphi$ не дает всплесков с компактным носителем. Очевидно, что сепарабельные многомерные всплески, полученные на основе всплесков Добеши, имеют компактньй носитель.

20. Неравенства Бернштейна. Регулярность масштабируюшей функции (19.7) позволяет рассматривать $\mathrm{KMA}\left\{V_{j}\right\}_{j \in \mathbb{Z}}$ не только в $L^{2}\left(\mathbb{R}^{n}\right)$, но и в других функциональных пространствах, например, в $L^{p}\left(\mathbb{R}^{n}\right), p \in[1, \infty]$.

ЛЕмма 20.1. Пусть ортогональная масштабирующая функиия $\varphi$ удовлетворяет (19.7). Тогда существуют две константы $c_{2}>c_{1}>0$ такие, что для любого $p \in[1, \infty]$ и любой конечной суммы $f(x)=\sum_{k} \alpha(k) \varphi(x-k)$ выполнень неравенства

$$
c_{1}\|f\|_{p} \leqslant\left(\sum_{k}|\alpha(k)|^{p}\right)^{1 / p} \leqslant c_{2}\|f\|_{p} .
$$


ДокАЗАТЕЛЬСтво. Начнем с крайних случаев. При $p=\infty$

$$
|f(x)| \leqslant \sum_{k}|\alpha(k)||\varphi(x-k)| \leqslant \sup _{k}|\alpha(k)| C(\varphi),
$$

где $C(\varphi):=\sup _{x \in \mathbb{R}^{n}} \sum_{k \in \mathbb{Z}}|\varphi(x-k)|$. В другую сторону воспользуемся тем, что $\alpha(k)=\int_{\mathbb{R}^{n}} f(x) \bar{\varphi}(x-k) d x$, поэтому $|\alpha(k)| \leqslant\|f\|_{\infty}\|\varphi\|_{1}$.

Случай $p=1$ разбирается так же:

$$
\begin{gathered}
\int_{\mathbb{R}^{n}}|f(x)| d x \leqslant \int_{\mathbb{R}^{n}} \sum_{k}|\alpha(k)||\varphi(x-k)| d x \leqslant \sum_{k}|\alpha(k)|\|\varphi\|_{1} ; \\
\sum_{k}|\alpha(k)| \leqslant \int_{\mathbb{R}^{n}}|f(x)| \sum_{k}|\varphi(x-k)| d x \leqslant C(\varphi)\|f\|_{1} .
\end{gathered}
$$

Рассмотрим общий случай. Пусть $q-$ сопряженньй показатель к $p$, т.е. $1 / p+1 / q=1$. Запишем $|\varphi(x-k)|=|\varphi(x-k)|^{1 / p}|\varphi(x-k)|^{1 / q}$, что дает

$$
\begin{aligned}
|f(x)| & \leqslant \sum_{k}|\alpha(k)||\varphi(x-k)| \\
& \leqslant\left(\sum_{k}|\alpha(k)|^{p}|\varphi(x-k)|\right)^{1 / p}\left(\sum_{k}|\varphi(x-k)|\right)^{1 / q} \\
& \leqslant C(\varphi)\left(\sum_{k}|\alpha(k)|^{p}\right)^{1 / p},
\end{aligned}
$$

откуда следует левая часть неравенства (20.1). Для доказательства правой части используем то, что $\alpha(k)=\int_{\mathbb{R}^{n}} f(x) \bar{\varphi}(x-k) d x$, поэтому

$$
|\alpha(k)| \leqslant\left(\int_{\mathbb{R}^{n}}|f(x)|^{p}|\varphi(x-k)| d x\right)^{1 / p}\|\varphi\|_{1}^{1 / q} .
$$

Определим $V_{0}(p)$ как пересечение $V_{0} \cap L^{p}\left(\mathbb{R}^{n}\right)$ для $1 \leqslant p \leqslant 2$ и как замыкание $V_{0}$ по $L^{p}\left(\mathbb{R}^{n}\right)$-норме для $2 \leqslant p<\infty$. По лемме $20.1 f \in V_{0}(p)$ тогда и только тогда, когда $f(x)=\sum_{k \in \mathbb{Z}^{n}} \alpha(k) \varphi(x-k)$, где $\alpha(k) \in l^{p}\left(\mathbb{Z}^{n}\right)$.

Определим $V_{0}(\infty)$ как векторное пространство, чьи элементы могут быть записаны как $f(x)=\lim f_{m}(x)$, где предел является равномерньм на компактах и где $f_{m} \in V_{0}$ и $\sup _{m \geqslant 0}\left\|f_{m}\right\|_{\infty}<\infty$. Другими словами, $f \in V_{0}(\infty)$ тогда и только тогда, когда $f(x)=\sum_{k \in \mathbb{Z}^{n}} \alpha(k) \varphi(x-k)$, где $\alpha(k) \in l^{\infty}\left(\mathbb{Z}^{n}\right)$.

Наконец, определим $V_{j}(p): f(\cdot) \in V_{j}(p) \Leftrightarrow f\left(2^{-j} \cdot\right) \in V_{0}(p)$. Очевидно, что $V_{j}(p)$ вложено в $L^{p}\left(\mathbb{R}^{n}\right)$. 
ТеОрема 20.1 [2, с. 32]. Пусть $V_{j}, j \in Z,-r$-регулярный кратномасштабный анализ в $L_{2}\left(\mathbb{R}^{n}\right)$. Тогда существует константа $C$ такая, что для $1 \leqslant p \leqslant \infty$, $j \in \mathbb{Z}, f \in V_{j}(p) u|\alpha| \leqslant r$ выполнено неравенство

$$
\left\|\partial^{\alpha} f\right\|_{p} \leqslant C 2^{|\alpha| j}\|f\|_{p}
$$

ДокАЗАТЕЛЬство. Заменой переменной все сводится к случаю $j=0$. Пусть $f(x)=\sum_{k} \alpha(k) \varphi(x-k)$. Тогда $\left|\partial^{\alpha} f(x)\right| \leqslant \sum_{k}|\alpha(k)|\left|\partial^{\alpha} \varphi(x-k)\right|$. Повторение рассуждений доказательства левого неравенства (20.1) дает $\left\|\partial^{\alpha} f\right\|_{p} \leqslant C\left(\sum_{k}|\alpha(k)|^{p}\right)^{1 / p}$, что можно оценить, используя правое неравенство (20.1).

Напомним, что классическое неравенство Бернштейна утверждает, что для любого $\alpha \in \mathbb{N}^{n}\left\|\partial^{\alpha} f\right\|_{p} \leqslant R^{|\alpha|}\|f\|_{p}$, где $f$ - произвольная функция из $L^{p}\left(\mathbb{R}^{n}\right), 1 \leqslant p \leqslant \infty$, чье преобразование Фурье имеет носитель в шаре $|\omega| \leqslant R$.

Таким образом, теорема 20.1 показывает, что по своим дифференциальньм свойствам элементы $V_{j}$ близки к целым функциям экспоненциального типа $2^{j}$, их преобразование Фурье сосредоточено в полосе $\left[-2^{j}, 2^{j}\right]$.

21. Регулярные КМА и полиномы. Пусть $\left\{V_{j}\right\}_{j \in \mathbb{Z}}-r$-регулярньй кратномасштабный анализ в $L_{2}\left(\mathbb{R}^{n}\right),\left\{P_{j}: L^{2}(\mathbb{R}) \rightarrow V_{j}\right\}_{j \in \mathbb{Z}}-$ ортогональные проекторы. Очевидно, что

$$
P_{0} f(x)=\int_{\mathbb{R}^{n}} E(x, y) f(y) d y
$$

где $E(x, y):=\sum_{k \in \mathbb{Z}} \varphi(x-k) \bar{\varphi}(y-k), \varphi$ - ортогональная масштабируюшая функция. Для произвольного $j \in \mathbb{Z}$

$$
P_{j} f(x)=\int_{\mathbb{R}^{n}} E_{j}(x, y) f(y) d y,
$$

где $E_{j}(x, y):=2^{n j} E\left(2^{j} x, 2^{j} y\right)$. Перечислим простейшие свойства ядер.

ЛЕмма 21.1.

$$
\left|\partial_{x}^{\alpha} \partial_{y}^{\beta} E(x, y)\right| \leqslant C_{m}(1+|x-y|)^{-m}
$$

для любьх $m \in \mathbb{N},|\alpha| \leqslant r,|\beta| \leqslant r ;$

$$
\begin{gathered}
E(x+k, y+k)=E(x, y), \quad k \in \mathbb{Z}^{n} ; \\
\lim _{j \rightarrow \infty}\left\|P_{j} f-f\right\|_{L^{2}(\mathbb{R})}=0 .
\end{gathered}
$$

Для доказательства последнего свойства в функциональных пространствах, отличных от $L^{2}\left(\mathbb{R}^{n}\right)$, нам потребуется следующий фундаментальньй результат, показьваюший, что полиномы степени не вьше $r$ инвариантны относительно проекторов $P_{j}$ $r$-регулярного КМА (точная формулировка в следствии 21.1 ниже). 
ТеОРема 21.1 [2, с. 33]. Для любого мультииндекса $\alpha \in \mathbb{N}^{n}$ с порядком $|\alpha| \leqslant r$ выполняется равенство

$$
\int_{\mathbb{R}^{n}} E(x, y) y^{\alpha} d y=x^{\alpha}
$$

СХемА ДОКАЗАТЕЛЬСТВА. В доказательстве существенную роль играет вложение $V_{0} \subset V_{j}, j \in \mathbb{N}$, которое на язьке проекторов преврашается в равенство $P_{0}=P_{j} P_{0}$, или, в терминах ядер:

$$
E(x, y)=2^{n j} \int_{\mathbb{R}^{n}} E\left(2^{j} x, 2^{j} u\right) E(u, y) d u
$$

Рассмотрим сначала случай $r=0$. Надо доказать, что

$$
\int_{\mathbb{R}^{n}} E(x, y) d y=1 \quad \forall x \in \mathbb{R}^{n}
$$

Свойство (21.2) имеет вид:

$$
|E(x, y)| \leqslant C_{m}\left(1+|x-y|^{-m}\right) \quad \forall m \in \mathbb{N} .
$$

Пусть $\mu_{0}(x):=\int_{\mathbb{R}^{n}} E(x, y) d y$. В силу $(21.3) \mu_{0}$ является $\mathbb{Z}^{n}$-периодической функцией:

$$
\mu_{0}(x+k)=\mu_{0}(x) \quad \forall k \in \mathbb{Z}^{n} .
$$

Равенство (21.6) получается предельным переходом из (21.5) при помоши следующего результата.

ЛЕмма $21.2\left[2\right.$, с. 34]. Для любого $y \in \mathbb{R}^{n}$ и для почти всех $x \in \mathbb{R}^{n}$ имеем

$$
\lim _{j \rightarrow \infty}\left\{2^{n j} \int_{\mathbb{R}^{n}} E\left(2^{j} x, 2^{j} u\right) E(u, y) d u-\mu_{0}\left(2^{j} x\right) E(x, y)\right\}=0 .
$$

Доказательство этой леммы основано на свойствах точек Лебега.

Из (21.9) с помощью (21.5) получаем, что для почти всех $x \in \mathbb{R}^{n}$

$$
\lim _{j \rightarrow \infty}\left(1-\mu_{0}\left(2^{j} x\right)\right) E(x, y)=0 \quad \forall y \in \mathbb{R}^{n} .
$$

Умножая (21.10) на $f \in V_{0}$ и интегрируя, имеем

$$
\lim _{j \rightarrow \infty} \mu_{0}\left(2^{j} x\right) f(x)=f(x) \quad \forall f \in V_{0}
$$

Заменой переменных получаем то же самое для $f \in V_{j_{0}}, j_{0} \geqslant 0$. Так как $\mu_{0}$ принадлежит $L_{\infty}\left(\mathbb{R}^{n}\right)$, сходимость в $(21.11)$ имеет место в $L_{2}\left(\mathbb{R}^{n}\right)$. 
Из (19.2) следует, что (21.11) имеет место для любой $f \in L_{2}\left(\mathbb{R}^{n}\right)$. В частности, полагая $f=\kappa_{[0,1]^{n}}$ и используя периодичность $\mu_{0}($ см. $(21.8))$, имеем

$$
\int_{[0,1]^{n}}\left|1-\mu_{0}(x)\right|^{2} d x=\int_{[0,1]^{n}}\left|1-\mu_{0}\left(2^{j} x\right)\right|^{2} d x \rightarrow 0 \text { при } j \rightarrow \infty .
$$

Откуда следует, что $\mu_{0}(x)=1$.

Для $r=0$ результат можно доказать проще, однако приведенное доказательство без больших изменений переносится на $r \geqslant 1$.

Разложим $E(u, y)$ в ряд Тейлора по степеням $u-x$ для того, чтобы оценить

$$
2^{n j} \int E\left(2^{j} x, 2^{j} u\right) E(u, y) d u
$$

Имеем

$$
E(u, y)=\sum_{0 \leqslant|\alpha|<r} \frac{(u-x)^{\alpha}}{\alpha !} \partial_{x}^{\alpha} E(x, y)+R(u, x, y)
$$

где

$$
\begin{gathered}
R(u, x, y):=\sum_{|\alpha|=r} \frac{(u-x)^{\alpha}}{\alpha !} S_{\alpha}(u, x, y), \\
S_{\alpha}(u, x, y):=r \int_{0}^{1} \partial_{x}^{\alpha} E(t u+(1-t) x, y)(1-t)^{r-1} d t .
\end{gathered}
$$

Сделаем замену $u=x+2^{-j} s$, которая преврашает $S_{\alpha}(u, x, y)$ в

$$
S_{\alpha}^{(j)}(s, x, y)=r \int_{0}^{1} \partial_{x}^{\alpha} E\left(x+t 2^{-j} s, y\right)(1-t)^{r-1} d t .
$$

Обозначим

$$
\mu_{\alpha}(x):=\frac{1}{\alpha !} \int_{\mathbb{R}^{n}} E(x, u)(u-x)^{\alpha} d u .
$$

Функция $\mu_{\alpha}(x)$ является непрерьвной и $\mathbb{Z}^{n}$-периодической (см. (21.8)). Подставляя $(21.13),(21.14)$ в $(21.12)$ и в $(21.5)$, получим, что

$$
\begin{aligned}
& 2^{n j} \int_{\mathbb{R}^{n}} E\left(2^{j} x, 2^{j} u\right) E(u, y) d u \\
& =\sum_{0 \leqslant \alpha<r} 2^{-j|\alpha|} \mu_{\alpha}\left(2^{j} x\right) \partial_{x}^{\alpha} E(x, y) \\
& \quad+r 2^{-j r} \sum_{|\alpha|=r} \int_{\mathbb{R}^{n}} \int_{0}^{1} E\left(2^{j} x, 2^{j} x+s\right) \frac{s^{\alpha}}{\alpha !} \partial_{x}^{\alpha} E\left(x+t 2^{-j} s, y\right)(1-t)^{r-1} d t d s \\
& \quad=E(x, y) .
\end{aligned}
$$


Доказательство того, что $\mu_{\alpha}(x)=0$, проводится индукцией по $p:=|\alpha| \in[1, r]$. Разберем случай $p=1$. В $(21.15) E(x, y)$ сокрашается, так как $\mu_{0}(x)=1$. Поэтому

$$
\sum_{1 \leqslant|\alpha| \leqslant r-1} 2^{-j|\alpha|} \mu_{\alpha}\left(2^{j} x\right) \partial_{x}^{\alpha} E(x, y)+O\left(2^{-j r}\right)=0,
$$

где оценка $O$ равномерна по $x$ и $y$. Умножим $(21.16)$ на $2^{j}$ и перейдем к пределу:

$$
\lim _{j \rightarrow \infty} \sum_{|\alpha|=1} \mu_{\alpha}\left(2^{j} x\right) \partial_{x}^{\alpha} E(x, y)=0
$$

Теперь мы повторяем анализ, проделанньй для случая $r=0$. Умножим (21.17) на $f \in V_{0}$ и проинтегрируем:

$$
\lim _{j \rightarrow \infty} \sum_{|\alpha|=1} \mu_{\alpha}\left(2^{j} x\right) \partial^{\alpha} f=0
$$

Пусть $g$ - непрерывная $\mathbb{Z}^{n}$-периодическая функция. Умножим (21.18) на произведение $g\left(2^{j} x\right) h(x)$, где $h \in D\left(\mathbb{R}^{n}\right)$, и проинтегрируем. Перейдем к пределу по $j \rightarrow \infty$ и воспользуемся хорошо известной леммой.

ЛЕмма 21.3. Если $и(x) \in L^{\infty}\left(\mathbb{R}^{n}\right)$ является $\mathbb{Z}^{n}$-периодической функиией и $v(x) \in L^{1}\left(\mathbb{R}^{n}\right), m o$

$$
\lim _{N \rightarrow \infty} \int_{\mathbb{R}^{n}} u(N x) v(x) d x=\left(\int u\right) \int_{\mathbb{R}^{n}} v(x) d x,
$$

где

$$
\int u=\int_{0}^{1} \cdots \int_{0}^{1} u\left(x_{1}, \ldots, x_{n}\right) d x_{1} \cdots d x_{n} .
$$

Окончательно получим, что

$$
\sum_{|\alpha|=1}\left(\int g \mu_{\alpha}\right)\left\langle\partial^{\alpha} f, h\right\rangle=0
$$

Полагая $c_{\alpha}=\int g \mu_{\alpha}$, из (21.20) получаем, что векторное поле $\sum_{|\alpha|=1} c_{\alpha} \partial^{\alpha}$ аннулирует любую функцию из $V_{0}$. Заменой переменной устанавливается, что аннигиляция имеет место для любой функшии из $V_{j}, j \in \mathbb{N}$. Учитывая (19.1), получаем это свойство (в смысле обобщенных функций) для всех $f \in L^{2}\left(\mathbb{R}^{n}\right)$. Таким образом, $c_{\alpha}=0$ для любой функции $g$, что означает, что $\mu_{\alpha}=0$ при $|\alpha|=1$.

Для $p=|\alpha|<r$ доказательство проходит без каких-либо изменений. Мы получаем, что $\mu_{\alpha}=0$ при $|\alpha|<r$.

Остается разобраться со случаем $|\alpha|=r$. Равенство (21.15) преврашается в

$$
r \sum_{|\alpha|=r} \int_{\mathbb{R}^{n}} \int_{0}^{1} E\left(2^{j} x, 2^{j} x+s\right) \frac{s^{\alpha}}{\alpha !} \partial_{x}^{\alpha} E\left(x+t 2^{-j} s, y\right)(1-t)^{r-1} d t d s=0 .
$$


Воспользуемся тем, что $\partial_{x}^{\alpha} E(x, y) \in L^{\infty}(d x)$ для любого фиксированного $y$. Почти все точки $x \in \mathbb{R}^{n}$ являются точками Лебега рассматриваемой функции. Если $x-$ точка Лебега, то в (21.21) $\partial_{x}^{\alpha} E\left(x+t 2^{-j} s, y\right)$ можно заменить на $\partial_{x}^{\alpha} E(x, y)$. Таким образом, предел по $j$

$$
\begin{aligned}
S_{j}(x, y) & :=r \sum_{|\alpha|=r} \int_{\mathbb{R}^{n}} \int_{0}^{1} E\left(2^{j} x, 2^{j} x+s\right) \frac{s^{\alpha}}{\alpha !} \partial_{x}^{\alpha} E(x, y)(1-t)^{r-1} d t d s \\
& =r \sum_{|\alpha|=r} \int_{\mathbb{R}^{n}} E\left(2^{j} x, 2^{j} x+s\right) \frac{s^{\alpha}}{\alpha !} \partial_{x}^{\alpha} E(x, y) d s \int_{0}^{1}(1-t)^{r-1} d t \\
& =\sum_{|\alpha|=r} \mu_{\alpha}\left(2^{j} x\right) \partial_{x}^{\alpha} E(x, y)
\end{aligned}
$$

равен 0 для любого $y \in \mathbb{R}^{n}$ и для почти всех $x \in \mathbb{R}^{n}$. Функции $S_{j}(x, y)$ удовлетворяют равномерной оценке $\left|S_{j}(x, y)\right| \leqslant C_{m}(1+|x-y|)^{-m}$. Умножая на $f \in V_{0}$ и интегрируя, находим, что $\sum_{|\alpha|=r} \mu_{\alpha}\left(2^{j} x\right) \partial^{\alpha} f$ сходится к 0 почти всюду и в $L^{2}\left(\mathbb{R}^{n}\right)$, так как можно применить теорему Лебега. Так же как и в случае $s<r$, заключаем, что все коэффициенты $\mu_{\alpha}$ обнуляются при $|\alpha|=r$.

$$
\text { Итак, доказано, что } \int_{\mathbb{R}^{n}} E(x, y)(x-y)^{\alpha} d y=0 \text { для } 1 \leqslant|\alpha| \leqslant r \text { и } \int_{\mathbb{R}^{n}} E(x, y) d y=1 .
$$

Отсюда следует, что $\int_{\mathbb{R}^{n}} E(x, y) y^{\alpha} d y=x^{\alpha}$ при $|\alpha| \leqslant r$. Действительно, запишем

$$
\frac{y^{\alpha}}{\alpha !}=\sum_{\beta+\gamma=\alpha} \frac{(y-x)^{\beta}}{\beta !} \frac{x^{\gamma}}{\gamma !}
$$

и, после интегрирования по $y$, все члены в правой части обнуляются, за исключением $\beta=0$ и $\gamma=\alpha$.

Теорема 21.1 может быть переформулирована, если расширить область определения проекторов $P_{j}$ на объединение пространств $L^{2}\left(\mathbb{R}^{n},(1+|x|)^{-m} d x\right), m \in \mathbb{N}$. Такое расширение возможно, так как ядра проекторов $P_{j}$ убывают быстрее любой степени при $|y-x|$, стремящемся к бесконечности. Пространство образов теперь будет отличаться от $V_{j}$, но будет полным по норме $L^{2}\left(\mathbb{R}^{n},(1+|x|)^{-m} d x\right)$.

СЛЕДСТВИЕ 21.1. Пусть $V_{j}-r$-регулярный кратномасштабный анализ в $L_{2}\left(\mathbb{R}^{n}\right)$ и пусть $P_{j}: L^{2}\left(\mathbb{R}^{n}\right) \rightarrow V_{j}$ - ортогональный проектор на $V_{j}$. Тогда $P_{j}(M)=M$ для любого полинома $M$ степени меньше или равной $r$.

Значение теоремы 21.1 состоит в том, что она устанавливает сходство проекторов $P_{j}$ с операторами свертки. Последние коммутируют с операторами частичного дифференцирования, тогда как операторы $P_{j}$ коммутируют приближенно с $\partial^{\alpha}$ при $|\alpha| \leqslant r$.

Зафиксируем функцию $g \in D\left(\mathbb{R}^{n}\right)$ с интегралом, равньм 1 . Обозначим через $g_{j}$ функцию $2^{n j} g\left(2^{j} x\right)$ и через $G_{j}$ - оператор свертки с $g_{j}, j \in \mathbb{Z}$. Теорема 21.1 позволяет сравнить $\partial^{\alpha} P_{j}$ с $G_{j} \partial^{\alpha}$ для мультииндексов $\alpha$ с $|\alpha| \leqslant r$. 
ТеОРема 21.2. Пусть $V_{j}, j \in \mathbb{Z},-$-регулярный кратномасштабный анализ в $L^{2}\left(\mathbb{R}^{n}\right)$. Тогда для любого мультииндекса $\alpha$ c $|\alpha| \leqslant r$ существуют функиии $R^{(\alpha, \beta)}(x, y) \in L^{\infty}\left(\mathbb{R}^{n}, \mathbb{R}^{n}\right)$, заиндексированнье мультииндексами $\beta$ с $|\beta|=|\alpha| u$ удовлетворяющие неравенствам

$$
\left|R^{(\alpha, \beta)}(x, y)\right| \leqslant C_{m}(1+|x-y|)^{-m}
$$

для любого $m \in \mathbb{N}$. Эти функиии удовлетворяют такжсе равенству

$$
\int_{\mathbb{R}^{n}} R^{(\alpha, \beta)}(x, y) d y=0
$$

тождественно по $x$ и определяют для любого $j \in \mathbb{Z}$ операторь

$$
\mathrm{R}_{j}^{(\alpha, \beta)} f(x):=2^{n j} \int_{\mathbb{R}^{n}} R^{(\alpha, \beta)}\left(2^{j} x, 2^{j} y\right) f(y) d y
$$

для которых верно следующее тождество:

$$
\partial^{\alpha} P_{j}=G_{j} \partial^{\alpha}+\sum_{|\beta|=|\alpha|} \mathrm{R}_{j}^{(\alpha, \beta)} \partial^{\beta},
$$

əде $\partial^{\alpha}=\left(\partial / \partial x_{1}\right)^{\alpha_{1}} \cdots\left(\partial / \partial x_{n}\right)^{\alpha_{n}}$.

ДокАЗАТЕЛЬСтво. Ясно, что (21.25) достаточно доказать для $j=0$. Общий случай получается заменой переменных.

Зафиксировав $x$, рассмотрим $\partial_{x}^{\alpha} E(x, y)$ как функцию по $y$. По теореме 21.1 имеем $\int_{\mathbb{R}^{n}} \partial_{x}^{\alpha} E(x, y) y^{\beta} d y=0$ при $|\beta| \leqslant|\alpha|$ и $\beta \neq \alpha$, тогда как $\int_{\mathbb{R}^{n}} \partial_{x}^{\alpha} E(x, y) y^{\alpha} d y=\alpha !$.

Заметим теперь, что ядро $g(x-y)$ оператора $G:=G_{0}$ имеет такие же свойства: $\int_{\mathbb{R}^{n}} \partial_{x}^{\alpha} g(x-y) y^{\beta} d y=0$ при $|\beta| \leqslant|\alpha|$ и $\beta \neq \alpha, \int_{\mathbb{R}^{n}} \partial_{x}^{\alpha} g(x-y) y^{\alpha} d y=\alpha !$.

Рассмотрим разность $R^{\alpha}(x, y):=\partial_{x}^{\alpha} E(x, y)-\partial_{x}^{\alpha} g(x-y)$, для которой

$$
\int_{\mathbb{R}^{n}} R^{\alpha}(x, y) y^{\beta} d y=0 \text { при }|\beta| \leqslant|\alpha| .
$$

Рассмотрим $f^{\alpha}(x, y):=R^{\alpha}(x, x+y)=\partial_{x}^{\alpha} E(x, x+y)-\partial_{x}^{\alpha} g(-y)$ как функцию от $y$ при фиксированном $x$. Эта функция принадлежит пространству $S_{r}\left(\mathbb{R}^{n}\right)$, которое определяется следуюшим образом.

ОПРЕДЕЛЕНИЕ 21.1. $S_{r}\left(\mathbb{R}^{n}\right)$ - это векторное пространство функций $f$, удовлетворяющих неравенствам $\left|\partial^{\alpha} f(x)\right| \leqslant C_{m}(1+|x|)^{-m}$ для любого $m \in \mathbb{N}$ и любого мультииндекса $\alpha \in \mathbb{N}^{n}$ с $|\alpha| \leqslant r$. 
Лемма 21.4. Пусть $0 \leqslant s \leqslant r$. Если $f$ принадлежит $S_{r}\left(\mathbb{R}^{n}\right)$ и удовлетворяет условиям $\int_{\mathbb{R}^{n}} f(x) x^{\alpha} d x=0$ для любого $\alpha \in \mathbb{N}^{n} c|\alpha| \leqslant r$, то

$$
f(x)=\sum_{|\alpha|=s} \partial^{\alpha} f_{\alpha}(x)
$$

где $f_{\alpha} \in S_{r}\left(\mathbb{R}^{n}\right) u \int_{\mathbb{R}^{n}} f_{\alpha}(x) d x=0$ для любого $\alpha \in \mathbb{N}^{n} c|\alpha|=s$.

Доказательство леммы проводится индукцией по размерности $n$. При $n=1$ $f_{s}$ является первообразной для $f$ порядка $s$, которая стремится к 0 на бесконечности.

Применим лемму к функции $f^{\alpha}(x, y)$, которую будем рассматривать как функцию по $y$, зависяшую от параметра $x$. Получаем, что

$$
\partial_{x}^{\alpha} E(x, y)=(-1)^{|\alpha|} \partial_{y}^{\alpha} g(x-y)+\sum_{|\beta|=|\alpha|} \partial_{y}^{\beta} R^{(\alpha, \beta)}(x, y)
$$

где $\left|R^{(\alpha, \beta)}(x, y)\right| \leqslant C_{m}(1+|x-y|)^{-m}$ для любых $m \in \mathbb{N}$. Более того,

$$
\int_{\mathbb{R}^{n}} R^{(\alpha, \beta)}(x, y) d y=0
$$

для любого $x$. Интегрирование по частям показьвает, что ядро оператора $G_{0} \partial^{\alpha}$ совпадает с $(-1)^{|\alpha|} \partial_{y}^{\alpha} g(x-y)$.

ЗАмЕчАНИЕ 21.1. Функции $f_{\alpha}$ из леммы 21.4 линейно и непрерывно зависят от $f$. Например, если $f$ является непрерьвной функцией некоторых параметров, то то же будет верно для функций $f_{\alpha}$. Это означает, что функции $R^{(\alpha, \beta)}$ будут непрерьвными по всем переменным, если масштабирующая функция $\varphi$ принадлежит $C^{r}\left(\mathbb{R}^{n}\right)$.

ЗАмечАниЕ 21.2. Аналогичньми рассуждениями доказьвается, что

$$
P_{j+1}-P_{j}=2^{-j r} \sum_{|\beta|=r} \mathrm{R}_{j}^{(\beta)} \partial^{\beta}
$$

где $\mathrm{R}_{j}^{(\beta)}$ определяются ядрами $2^{n j} R^{(\beta)}\left(2^{j} x, 2^{j} y\right)$ и $R^{(\beta)}$ удовлетворяют свойствам, аналогичным свойствам $R^{(\alpha, \beta)}$.

\section{2. КМА в пространствах Соболева.}

Теорема 22.1. Пусть $r \in \mathbb{N} u V_{j}, j \in \mathbb{Z}$, является $r$-регулярным кратномасштабным анализом в $L_{2}\left(\mathbb{R}^{n}\right)$. Если $f$ принадлежит пространству Соболева $W_{2}^{s}\left(\mathbb{R}^{n}\right) u-r \leqslant s \leqslant r, \operatorname{mo~} P_{j}(f)$ сходится $\kappa f$ в $W_{2}^{s}\left(\mathbb{R}^{n}\right)$-норме.

В доказательстве этой теоремы используется следующая лемма. 
Лемма 22.1. Пусть $K(x, y)$ - функиия из $L^{\infty}\left(\mathbb{R}^{n} \times \mathbb{R}^{n}\right)$, удовлетворяющая неравенству $|K(x, y)| \leqslant C(1+|x-y|)^{-n-1}, u$

$$
T_{\lambda}: L^{p}\left(\mathbb{R}^{n}\right) \rightarrow L^{p}\left(\mathbb{R}^{n}\right), \quad 1 \leqslant p \leqslant \infty, \quad \lambda>0
$$

операторье с ядрами $\lambda^{n} K(\lambda x, \lambda y), T_{\lambda} f(x)=\lambda^{n} \int_{\mathbb{R}^{n}} K(\lambda x, \lambda y) f(y) d y$. Ecли

$$
\int_{\mathbb{R}^{n}} K(x, y) d y=0
$$

тождественно по х $и 1 \leqslant p<\infty$, то

$$
\lim _{\lambda \rightarrow \infty}\left\|T_{\lambda}(f)\right\|_{p}=0 \quad \text { для } \quad f \in L^{p}
$$

Если $\int_{\mathbb{R}^{n}} K(x, y) d y=1$ тождественно по $x$ и $1 \leqslant p<\infty$, то

$$
\lim _{\lambda \rightarrow \infty}\left\|T_{\lambda}(f)-f\right\|_{p}=0 \quad \text { для } \quad f \in L^{p} .
$$

При $p=\infty$ предположим в дополнение, что $f$ является равномерно непрерывной и что $K(x, y)$ является непрерывной функцией по $x$ при каждом фиксированном у. Тогда, если $\int_{\mathbb{R}^{n}} K(x, y) d y=1 u f \in L^{\infty}\left(\mathbb{R}^{n}\right)$, mо

$$
\lim _{\lambda \rightarrow \infty}\left\|T_{\lambda}(f)-f\right\|_{\infty}=0
$$

ДоказАтельство. Во-первых, заметим, что норма оператора $T_{\lambda}: L^{p}\left(\mathbb{R}^{n}\right) \rightarrow$ $L^{p}\left(\mathbb{R}^{n}\right)$ является конечной и не зависит от $\lambda$.

Если $1 \leqslant p<\infty$, то достаточно рассмотреть плотное подмножество в $L_{p}\left(\mathbb{R}^{n}\right)$. Пусть $f$ - непрерьвная функция с компактным носителем. Тогда $T_{\lambda} f(x)$ является $O\left(|x|^{-n-1}\right)$ при $|x| \rightarrow \infty$ равномерно по $\lambda \geqslant 1$. Так как $\int_{\mathbb{R}^{n}} K(x, y) d y=0$, то

$$
T_{\lambda} f(x)=\lambda^{n} \int_{\mathbb{R}^{n}} K(\lambda x, \lambda y) f(y) d y=\lambda^{n} \int_{\mathbb{R}^{n}} K(\lambda x, \lambda y)(f(y)-f(x)) d y,
$$

что меншше, чем

$$
C \int_{\mathbb{R}^{n}}(1+|y|)^{-n-1} w\left(\lambda^{-1}|y|, f\right) d y=: \epsilon(\lambda)
$$

где $w$ - модуль непрерьвности $f$. Из теоремы Лебега о доминируемой сходимости следует, что $\lim _{\lambda \rightarrow \infty} \epsilon(\lambda)=0$. Таким образом, функции $T_{\lambda} f(x)$ сходятся к 0 равномерно по $x$ и равномерно по $\lambda$ являются $O\left(|x|^{-n-1}\right)$ при $|x| \rightarrow \infty$. Значит, они сходятся к 0 в $L^{p}\left(\mathbb{R}^{n}\right)$-норме.

Второй случай леммы, когда $\int_{\mathbb{R}^{n}} K(x, y) d y=1$, является следствием первого. Пусть $g$ - непрерьвная функция с компактньм носителем, чей интеграл равен 1. Заменим $K(x, y)$ на $R(x, y):=K(x, y)-g(x-y)$ и применим первый случай. 
ДоКАЗАТЕЛЬСТВо ТЕОРЕмЫ 22.1. Пусть $s=r$. Надо доказать, что для $f \in$ $W_{2}^{s}\left(\mathbb{R}^{n}\right)$ и $|\alpha| \leqslant r$

$$
\lim _{j \rightarrow \infty}\left\|\partial^{\alpha} P_{j}(f)-\partial^{\alpha} f\right\|_{2}=0
$$

По теореме 21.2 имеем $\partial^{\alpha} P_{j}=G_{j} \partial^{\alpha}+\sum_{|\beta|=|\alpha|} \mathrm{R}_{j}^{(\alpha, \beta)} \partial^{\beta}$, поэтому достаточно применить лемму 22.1.

Если $s$ не целое и $0<s<r$, то заметим, что операторы $P_{j}: W_{2}^{s} \rightarrow W_{2}^{s}$ равномерно ограничены. Это следует из крайних случаев $s=0, s=r$ и следующей интерполящионной теоремы.

УТВЕРЖДЕНИЕ 22.1. Любой непрерывный оператор $T: L^{2} \rightarrow L^{2}$, чье сужение на пространство Соболева $W_{2}^{r}$ является непрерывным линейным оператором из $W_{2}^{r}$ в $W_{2}^{r}$, является также непрерывным оператором из $W_{2}^{s}$ в $W_{2}^{s}$ для $0<s<r$. Если нормы $T$ как оператора в $L^{2}$ u $W_{2}^{r}$ обозначить $C_{0}$ и $C_{1}$ соответственно, то норма $T$ как оператора в $W_{2}^{s}, 0<s<r$, не превосходит $\max \left(C_{0}, C_{1}\right)$.

Из равномерной ограниченности операторов $P_{j}$ и плотности $W_{2}^{r}\left(\mathbb{R}^{n}\right)$ в $W_{2}^{s}\left(\mathbb{R}^{n}\right)$ следует утверждение теоремы.

Для доказательства теоремы 22.1 при $-r \leqslant s \leqslant 0$ нужно сначала определить $P_{j}$ на $W_{2}^{s}\left(\mathbb{R}^{n}\right)$. Заметим, что $L^{2}\left(\mathbb{R}^{n}\right)$ плотно в $W_{2}^{s}\left(\mathbb{R}^{n}\right)$. Если $f$ и $g$ принадлежат $L_{2}\left(\mathbb{R}^{n}\right)$, то $\left(P_{j}(f), g\right)=\left(f, P_{j}(g)\right)$. Теперь мы предположим, что $f$ принадлежит $W_{2}^{s}\left(\mathbb{R}^{n}\right)$, где $-r \leqslant s \leqslant 0$, и что $f_{m}$ - это последовательность функций $L^{2}\left(\mathbb{R}^{n}\right)$, сходяшаяся к $f$ в $W_{2}^{s}\left(\mathbb{R}^{n}\right)$-норме. Тогда для $t=-s$ и $g \in W_{2}^{t}\left(\mathbb{R}^{n}\right)$ имеем

$$
\left(P_{j}\left(f_{m}\right), g\right)=\left(f_{m}, P_{j}(g)\right) \rightarrow\left(f, P_{j}(g)\right) \text { при } m \rightarrow \infty
$$

Поэтому мы определяем $P_{j}(f) \in W_{2}^{s}\left(\mathbb{R}^{n}\right)$ следуюшим образом:

$$
\left(P_{j}(f), g\right)=\left(f, P_{j}(g)\right) .
$$

Так как $P_{j}^{2}=P_{j}$, то $\left(P_{j}(f), P_{j}(g)\right)=\left(P_{j}(f), g\right)$.

По двойственности операторы $P_{j}: W_{2}^{s}\left(\mathbb{R}^{n}\right) \rightarrow W_{2}^{s}\left(\mathbb{R}^{n}\right)$ равномерно ограничены. Кроме того, $L^{2}\left(\mathbb{R}^{n}\right)$ плотно в $W_{2}^{s}\left(\mathbb{R}^{n}\right)$ и $P_{j}(f)$ сходится к $f$ для $f \in L^{2}\left(\mathbb{R}^{n}\right)$. Из этого следует, что $P_{j}(f)$ сходится к $f$ в $W_{2}^{s}\left(\mathbb{R}^{n}\right)$-норме. Стандарным способом доказывается, что $\lim _{j \rightarrow \infty} P_{j}(f)=f$ для распределений $f$, принадлежаших $W_{2}^{s}\left(\mathbb{R}^{n}\right)$ при $-r \leqslant s<0$.

ЗАмечАниЕ 22.1. Теорему 22.1 можно обобшить на пространства $W_{p}^{s} \mathrm{c}-r \leqslant s \leqslant r$ и $1<p<\infty$. Для функций и распределений $f \in W_{p}^{s}$ проекции $P_{j}(f)$ сходятся к $f$ в $W_{p}^{s}$-норме.

Случай $p=\infty$ требует отдельного рассмотрения. Определим пространство $W_{\infty}^{m}$, $m \in \mathbb{N}$, условием, что $\partial^{\alpha} f$ принадлежат $L^{\infty}\left(\mathbb{R}^{n}\right)$ для любых $\alpha$ с $|\alpha| \leqslant m$. Тогда для $f \in W_{\infty}^{m}, 0 \leqslant m \leqslant r$, последовательность $P_{j}(f)$ равномерно ограничена в $W_{\infty}^{m}$ и слабо сходится к $f$, т.е.

$$
\int P_{j}(f) g d x \rightarrow \int f g d x \quad \forall g \in S\left(\mathbb{R}^{n}\right)
$$


Можно заменить $W_{\infty}^{m}\left(\mathbb{R}^{n}\right)$ на пространство $C_{\infty}^{m}\left(\mathbb{R}^{n}\right)$, определяемое условием, что все производные $\partial^{\alpha} f,|\alpha| \leqslant m$, ограничены и равномерно непрерывны на $\mathbb{R}^{n}$. Тогда для любой функции $f \in C_{\infty}^{m}\left(\mathbb{R}^{n}\right)$ проекции $P_{j}(f)$ сходятся к $f$ в $C_{\infty}^{m}\left(\mathbb{R}^{n}\right)$-норме при $0 \leqslant m<r$. Если масштабируюшая функция $\varphi$ принадлежит $C_{\infty}^{m}\left(\mathbb{R}^{n}\right)$ и быстро убывает на бесконечности вместе со всеми производными порядка $|\alpha| \leqslant r$, то сходимость имеет место и при $m=r$.

Если $\varphi \in C^{m}\left(\mathbb{R}^{n}\right)$ и имеет компактный носитель, то $P_{j}(f)$ сходятся к $f \in C^{m}\left(\mathbb{R}^{n}\right)$ в $C^{m}\left(\mathbb{R}^{n}\right)$-норме.

23. Операторы $Q_{j}=P_{j+1}-P_{j}$. Пусть $V_{j}, j \in \mathbb{Z},-r$-регулярный $\mathrm{KMA} \mathrm{в} L^{2}\left(\mathbb{R}^{n}\right)$ и $W_{j}$ - ортогональное дополнение $V_{j}$ до $V_{j+1}$. Ортогональный проектор из $L^{2}\left(\mathbb{R}^{n}\right)$ на $W_{j}$ равен $Q_{j}=P_{j+1}-P_{j}$. Ясно, что $f(\cdot) \in W_{0}$ тогда и только тогда, когда $f\left(2^{j} \cdot\right) \in W_{j}$. Кроме того,

$$
L^{2}\left(\mathbb{R}^{n}\right)=\bigoplus_{-\infty}^{\infty} W_{j}
$$

так как объединение $V_{j}$ плотно в $L^{2}\left(\mathbb{R}^{n}\right)$, а пересечение $V_{j}$ равно $\{0\}$. Любую функцию $f \in L^{2}\left(\mathbb{R}^{n}\right)$ можно разложить в ортогональный ряд

$$
f=\sum_{-\infty}^{\infty} Q_{j}(f)
$$

Покажем, что классические нормы пространств функций и распределений легко вычисляются с использованием этого разложения.

Распространим разложение $(23.1)$ на $L^{p}\left(\mathbb{R}^{n}\right), 1 \leqslant p \leqslant \infty$. Если $1 \leqslant p \leqslant 2$, то $W_{j}(p)$ определим как пересечение $W_{j}$ с $L^{p}\left(\mathbb{R}^{n}\right)$, что согласовано с определением $V_{j}(p)$ (см. раздел 20). Тогда $V_{j+1}(p)=V_{j}(p)+W_{j}(p)$, где сумма прямая, но не ортогональная. Операторы $P_{j}$ и $Q_{j}$, проектируюшие $V_{j+1}(p)$ на $V_{j}(p)$ и $W_{j}(p)$ соответственно, являются непрерьвными в $L^{p}\left(\mathbb{R}^{n}\right)$ (см. лемму 22.1). Кроме того, при $1<p \leqslant 2$ для любой функции $f \in L^{p}\left(\mathbb{R}^{n}\right)\left\|P_{j} f\right\|_{p} \rightarrow 0$ при $j \rightarrow-\infty$. Таким образом, функция $f \in L^{p}\left(\mathbb{R}^{n}\right)$ может быть единственным образом записана в виде

$$
f=\sum_{-\infty}^{\infty} q_{j}, \quad q_{j} \in W_{j}(p)
$$

где частные суммы сходятся к $f$ в $L_{p}\left(\mathbb{R}^{n}\right)$ при $1<p \leqslant 2$.

В случае $2 \leqslant p<\infty$ пространства $W_{j}(p)$ определяются как замыкание $W_{j}$ в $L^{p}\left(\mathbb{R}^{n}\right)$. Тогда $(23.3)$ распространяется на $L^{p}\left(\mathbb{R}^{n}\right), 2 \leqslant p<\infty$.

Для $p=\infty W_{j}(p)$ - это замыкание $W_{j}$ в $L^{\infty}\left(\mathbb{R}^{n}\right)$ с топологией $\sigma\left(L^{\infty}, L^{1}\right)$.

Следуюший результат дополняет и уточняет неравенства Бернштейна. 
ТеОрема 23.1. Пусть $V_{j}, j \in \mathbb{Z},-$-регулярный кратномасштабный анализ в $L^{2}\left(\mathbb{R}^{n}\right)$. Тогда существуют две константы $C_{2} \geqslant C_{1}>0$ такие, что для любого целого $s \in \mathbb{N}, s \in[0, r]$, для любого $p \in[1, \infty]$ и любой функиии $f \in W_{0}(p)$

$$
C_{1}\|f\|_{p} \leqslant \sum_{|\alpha|=s}\left\|\partial^{\alpha} f\right\|_{p} \leqslant C_{2}\|f\|_{p}
$$

Eсли $s$ не иелое $и 0<s<r$, mо

$$
C_{1}\|f\|_{p} \leqslant\left\|\Lambda^{s} f\right\|_{p} \leqslant C_{2}\|f\|_{p}
$$

əде $\Lambda=(-\Delta)^{1 / 2}$.

ДокАЗАТЕльство. Правая часть (23.4) полностью совпадает с (20.2). Для доказательства левой части обозначим через $Q(x, y)$ ядро проектора $Q_{0}=P_{1}-P_{0}$. Тогда очевидно, что $f(x)=\int_{\mathbb{R}^{n}} Q(x, y) f(y) d y$, если $f \in W_{0}$. С одной стороны, $|Q(x, y)| \leqslant C_{m}(1+|x-y|)^{-m}$ для любого $m \in \mathbb{N}$. C другой, $Q(x, y)=2^{n} E(2 x, 2 y)-$ $E(x, y)$, где $E(x, y)$ - ядро $P_{0}$, следовательно, по теореме 21.1

$$
\int_{\mathbb{R}^{n}} Q(x, y) y^{\alpha} d y=0 \text { при }|\alpha| \leqslant r .
$$

Используя лемму 21.4 , получим, что $Q(x, y)=\sum_{|\beta|=s} \partial_{y}^{\beta} Q_{\beta}(x, y)$, где функции $Q_{\beta}$ также удовлетворяют условиям $\left|Q_{\beta}(x, y)\right| \leqslant C_{m}^{\prime}(1+|x-y|)^{-m}$ для любого $m \in \mathbb{N}$. Таким образом,

$$
f(x)=\int_{\mathbb{R}^{n}} Q(x, y) f(y) d y=(-1)^{s} \sum_{|\beta|=s} \int_{\mathbb{R}^{n}} Q_{\beta}(x, y) \partial^{\beta} f(y) d y .
$$

Так как ядра $Q_{\beta}(x, y)$ определяют ограниченные операторы в $L^{p}\left(\mathbb{R}^{n}\right)$ при $1 \leqslant p \leqslant \infty$ (см. доказательство леммы 22.1), то левая часть (23.4) доказана.

Пусть $s-$ не целое и $m \in \mathbb{Z}$ такое, что $m<s<m+1$. Для $Q_{0}$ имеют место два представления $Q_{0}=\sum_{|\beta|=m} Q_{\beta} \partial^{\beta}=\sum_{|\beta|=m+1} Q_{\beta} \partial^{\beta}$, которые приводят к равенству $Q_{0}=G \Lambda^{s}$, где $G$ - оператор, который ограничен во всех $L_{p}\left(\mathbb{R}^{n}\right), 1 \leqslant p \leqslant \infty$. Действительно, определим оператор свертки $U$, соответствуюший мультипликатору $u(\xi) \in D\left(\mathbb{R}^{n}\right)$, который радиален, равен 1 при $|\xi| \leqslant 1 / 2$ и равен 0 при $|\xi| \geqslant 1$. Пусть $V:=I-U$. Тогда

$$
\begin{aligned}
Q_{0} & =Q_{0} U+Q_{0} V=\sum_{|\beta|=m+1} Q_{\beta} \partial^{\beta} \Lambda^{-s} U \Lambda^{s}+\sum_{|\beta|=m} Q_{\beta} \partial^{\beta} \Lambda^{-s} V \Lambda^{s} \\
& =\left(\sum_{|\beta|=m+1} Q_{\beta} \partial^{\beta} \Lambda^{-s} U+\sum_{|\beta|=m} Q_{\beta} \partial^{\beta} \Lambda^{-s} V\right) \Lambda^{s}=: G \Lambda^{s} .
\end{aligned}
$$

Непрерьвность $G$ в $L^{p}\left(\mathbb{R}^{n}\right)$ следует из непрерывности операторов $\partial^{\beta} \Lambda^{-s} U,|\beta|=$ $m+1$, и $\partial^{\beta} \Lambda^{-s} V,|\beta|=m$, в $L^{p}\left(\mathbb{R}^{n}\right)$, которые являются операторами свертки, соответствуюшими мультипликаторам $\xi^{\beta}|\xi|^{-s} u(\xi),|\beta|=m+1$, и $\xi^{\beta}|\xi|^{-s}(1-u(\xi))$, $|\beta|=m$, (с точностью до степени $i$ ). Эти функции являются преобразованиями Фурье интегрируемых функций, что завершает доказательство. 
СлЕДСТВИЕ 23.1. Если $1 \leqslant p \leqslant \infty u f$ принадлежит $W_{j}(p)$, то

$$
C_{1} 2^{j s}\|f\|_{p} \leqslant \sum_{|\alpha|=s}\left\|\partial^{\alpha} f\right\|_{p} \leqslant C_{2} 2^{j s}\|f\|_{p}
$$

при иелом $s \in[0, r]$.

В общем случае действительного $s \in[0, r]$

$$
C_{1} 2^{j s}\|f\|_{p} \leqslant\left\|\Lambda^{s} f\right\|_{p} \leqslant C_{2} 2^{j s}\|f\|_{p}
$$

ДокАЗАТЕльство. Для доказательства достаточно сделать замену переменных в (23.4) и (23.5).

Заметим, что при $j \in \mathbb{N} \Lambda^{s}$ можно заменить в $(23.7)$ на $(I-\Delta)^{s / 2}$. Ясно, что при $1 \leqslant p \leqslant \infty\left\|\Lambda^{s} f\right\|_{p} \leqslant C(s, n)\left\|(I-\Delta)^{s / 2} f\right\|_{p}$. Достаточно сравнить преобразования Фурье $\Lambda^{s} f$ и $(I-\Delta)^{s / 2} f$. С другой стороны, $\left\|(I-\Delta)^{s / 2} f\right\|_{p} \leqslant C^{\prime}(s, n)\left\|\left(I+\Lambda^{s}\right) f\right\|_{p}$ при $1 \leqslant p \leqslant \infty$.

УТВЕРЖДЕНИЕ 23.1. Если $-r \leqslant s \leqslant r, 1 \leqslant p \leqslant \infty u f \in W_{j}(p)$ для $j \in \mathbb{N}, m o$

$$
C_{1}^{\prime} 2^{j s}\|f\|_{p} \leqslant\left\|(I-\Delta)^{s / 2} f\right\|_{p} \leqslant C_{2}^{\prime} 2^{j s}\|f\|_{p}
$$

где $C_{2}^{\prime}>C_{1}^{\prime}>0-$ константы.

ДоКАЗАТЕльство. В доказательстве нуждается только случай $-r \leqslant s<0$, так как случай $0 \leqslant s \leqslant r$ рассмотрен в следствии 23.1. Замена переменной $x \mapsto 2^{j} x$ сводит доказательство к следуюшим неравенствам

$$
C_{1}^{\prime}\|f\|_{p} \leqslant\left\|(\epsilon I-\Delta)^{s / 2} f\right\|_{p} \leqslant C_{2}^{\prime}\|f\|_{p}
$$

для $f \in W_{0}(p)$ и $\epsilon:=4^{-j}$.

Для доказательства правой части воспользуемся тождеством $I=U+V$ (см. доказательство теоремы 23.1). Оператор $(\epsilon I-\Delta)^{s / 2}$ разлагается на сумму $(\epsilon I-\Delta)^{s / 2} U$ и $(\epsilon I-\Delta)^{s / 2} V$. Второе выражение - это оператор свертки, соответствующий мультипликатору $\left(\epsilon+|\omega|^{2}\right)^{s / 2}(1-u(\omega))$. Так как $u(\omega)=1$ при $|\omega| \leqslant 1 / 2$, сингулярности первого множителя в нуле уничтожаются за счет второго. Таким образом, оператор $(\epsilon I-\Delta)^{s / 2} V$ равномерно ограничен на $L^{p}\left(\mathbb{R}^{n}\right)$ при $1 \leqslant p \leqslant \infty$.

Для изучения действия $(\epsilon I-\Delta)^{s / 2} U$ на $W_{0}(p)$ используем воспроизводяшее ядро $Q(x, y)$ подпространства $W_{0}$. Тогда $f(x)=\int_{\mathbb{R}^{n}} Q(x, y) f(y) d y$ для $f \in W_{0}$. Оператор $Q_{0}$ является самосопряженным и тождество $Q_{0}=\sum_{|\beta|=r} Q_{\beta} \partial^{\beta}$ приводит к $Q_{0}=\sum_{|\beta|=r} \partial^{\beta} \widetilde{Q}_{\beta}$, где $\widetilde{Q}_{\beta}$ равномерно ограничены в $L^{p}\left(\mathbb{R}^{n}\right)$ при $1 \leqslant p \leqslant \infty$. Таким образом, имеем

$$
(\epsilon I-\Delta)^{s / 2} U f=\sum_{|\beta|=r}\left((\epsilon I-\Delta)^{s / 2} U \partial^{\beta}\right) \widetilde{Q}_{\beta} f
$$

и операторы $(\epsilon I-\Delta)^{s / 2} U \partial^{\beta}: L^{p}\left(\mathbb{R}^{n}\right) \rightarrow L^{p}\left(\mathbb{R}^{n}\right)$ равномерно ограничены в $L^{p}\left(\mathbb{R}^{n}\right)$ при $|\beta|=r>|s|$ и $0 \leqslant \epsilon \leqslant 1$, так как соответствуюшие мультипликаторы равны 
$\left(\epsilon+|\omega|^{2}\right)^{s / 2} u(\omega) \omega^{\beta}$ (с точностью до степени $i$ ) и совпадают с преобразованием Фурье интегрируемых функций. Правая часть неравенства (23.9) доказана.

Пусть $t=-s$. Тогда $f=E_{0}(f)=E_{0}(\epsilon I-\Delta)^{t / 2}(\epsilon I-\Delta)^{s / 2} f$. Операторы $E_{0}(\epsilon I-\Delta)^{t / 2}$ равномерно ограничены в $L^{p}\left(\mathbb{R}^{n}\right)$ при $1 \leqslant p \leqslant \infty$, так как сопряженные к ним операторы равномерно ограничены в $L^{p}\left(\mathbb{R}^{n}\right)$ в силу теоремы 20.1 .

Охарактеризуем пространства Соболева $W_{2}^{s}\left(\mathbb{R}^{n}\right)$ в терминах разложения (23.2).

ТЕОРема 23.2. Если $V_{j}, j \in \mathbb{N},-r$-регулярный кратномасштабный анализ в $L_{2}\left(\mathbb{R}^{n}\right)$ и $s \in(-r, r)$, то функиия $f$ принадлежст пространству Соболева $W_{2}^{s}\left(\mathbb{R}^{n}\right)$ тогда и только тогда, когда $P_{0}(f) \in L^{2}\left(\mathbb{R}^{n}\right)$ u $\left\|Q_{j} f\right\|_{2}=\epsilon_{j} 2^{-j s}$ для всех $j \in \mathbb{N}$, где $\left\{\epsilon_{j}\right\}_{0}^{\infty} \in l^{2}(\mathbb{N})$. Более того, $W_{2}^{s}$-норма $f$ әквивалентна сумме $L^{2}$-нормы $P_{0}(f)$ и $l^{2}(\mathbb{N})$-нормы последовательности $\epsilon_{j}$.

ДокАЗАТЕЛЬСтво. Из теоремы 20.1 следует, что $V_{0} \subset W_{2}^{s}\left(\mathbb{R}^{n}\right)$. Поэтому для доказательства достаточности нужно показать, что сумма функций $q_{j} \in W_{j}$ c $\left\|q_{j}\right\|_{2}=$ $\epsilon_{j} 2^{-j s}$, где $\epsilon_{j} \in l^{2}(\mathbb{N})$, принадлежит $W_{2}^{s}\left(\mathbb{R}^{n}\right)$. Зафиксируем положительное $\epsilon$, для которого $|s|+2 \epsilon \leqslant r$. Пространство $W_{2}^{s}$ является гильбертовым со скалярньм произведением $\left\langle(I-\Delta)^{s / 2} u,(I-\Delta)^{s / 2} v\right\rangle$. Обозначим через $\|\cdot\|_{s}$ соответствующую норму в $W_{2}^{s}$. Заметим, что

$$
\left|\left\langle(I-\Delta)^{s / 2} u,(I-\Delta)^{s / 2} v\right\rangle\right|=\left\langle(I-\Delta)^{s / 2+\epsilon} u,(I-\Delta)^{s / 2-\epsilon} v\right\rangle \leqslant\|u\|_{s+2 \epsilon}\|v\|_{s-2 \epsilon} .
$$

Поэтому

$$
\left\|\sum_{0}^{\infty} q_{j}\right\|_{s}^{2} \leqslant 2 \sum_{j<k} \sum_{j}\left\|q_{j}\right\|_{s+2 \epsilon}\left\|q_{k}\right\|_{s-2 \epsilon}+\sum_{j}\left\|q_{j}\right\|_{s}^{2}
$$

Из утверждения 23.1 следует, что $\left\|q_{j}\right\|_{\sigma} \asymp 2^{j \sigma} 2^{-j s} \epsilon_{j}$ для $\sigma \in[-r, r]$. Поэтому

$$
\left\|q_{j}\right\|_{s+2 \epsilon}\left\|q_{k}\right\|_{s-2 \epsilon} \leqslant C \epsilon_{j} \epsilon_{k} 4^{\epsilon(j-k)}
$$

Ряд $\sum \sum_{j<k} \epsilon_{j} \epsilon_{k} 4^{-\epsilon|j-k|}$ сходится, так как свертка $l^{1}$-последовательности $\left\{4^{-\epsilon|j-k|}\right\}$ с $l^{2}$-последовательностью является $l^{2}$-последовательностью (далее неравенство Коши).

Необходимость условий $P_{0}(f) \in L^{2}\left(\mathbb{R}^{n}\right)$ и $\left\|Q_{j} f\right\|_{2} \leqslant \epsilon_{j} 2^{-j s}$ для принадлежности $f$ к $W_{2}^{s}$ следует из достаточности условий для $t=-s$ и соображений двойственности.

СлЕДСТВИЕ 23.2. Если $-r<s<r, f \in W_{2}^{s}\left(\mathbb{R}^{n}\right) u g \in W_{2}^{-r}\left(\mathbb{R}^{n}\right), m o$

$$
\langle f, g\rangle=\left\langle P_{0} f, P_{0} g\right\rangle+\sum_{0}^{\infty}\left\langle Q_{j} f, Q_{j} g\right\rangle
$$

причем ряд в правой части сходится абсолютно.

ДокаЗАТЕльство. Действительно, $\left\|Q_{j}(f)\right\|_{2} \leqslant \epsilon_{j} 2^{-j s}$ и $\left\|Q_{j} g\right\|_{2} \leqslant \eta_{j} 2^{j s}$, поэтому $\left\langle Q_{j}(f), Q_{j}(g)\right\rangle \leqslant \epsilon_{j} \eta_{j}$ и ряд $\sum_{0}^{\infty} \epsilon_{j} \eta_{j}$ сходится абсолютно. 
24. Пространства Бесова. Если в определении 2.8 пространств Бесова положить $g_{j}:=f_{j+1}-f_{j}$, то

$$
f=f_{0}+\sum_{0}^{\infty} g_{j}
$$

где $\partial^{\alpha} g_{j} \leqslant \varepsilon_{j} 2^{(m-s) j}$ при $|\alpha|=m$ и $\left\|g_{j}\right\| \leqslant \varepsilon_{j} 2^{-j s}$. Очевидно, что верно и обратное.

Пусть $V_{j}, j \in \mathbb{Z},-r$-регулярный $\mathrm{KMA} \mathrm{в}^{2}\left(\mathbb{R}^{n}\right), P_{j}: L^{2}\left(\mathbb{R}^{n}\right) \rightarrow V_{j}$ - ортогональный проектор, $Q_{j}=P_{j+1}-P_{j}$.

УТВЕРЖДЕНИЕ 24.1. Пусть $0<s<r u f \in L^{p}\left(\mathbb{R}^{n}\right)$. Функиия $f$ принадлежит пространству Бесова $B_{p}^{s, q}\left(\mathbb{R}^{n}\right)$ тогда и только тогда, когда последовательность $\left\{2^{j s}\left\|Q_{j}(f)\right\|_{p}\right\}_{j \in \mathbb{N}}$ принадлежит $l^{q}(\mathbb{N})$. Более того, норма $f$ в $B_{p}^{s, q}\left(\mathbb{R}^{n}\right)$ әквивалентна сумме $l^{q}$-нормы этой последовательности и $\left\|P_{0}(f)\right\|_{p}$.

ДокАЗАТЕЛЬСтво. Достаточность следует из теоремы 20.1.

Необходимость. Из (24.1) следует, что $Q_{j}(f)=Q_{j}\left(f_{0}\right)+\sum_{k=0}^{\infty} Q_{j}\left(g_{k}\right)$. Для $k \geqslant j$ $\left\|Q_{j}\left(g_{k}\right)\right\|_{p} \leqslant C\left\|g_{k}\right\|_{p}$, так как операторы $Q_{j}: L^{p} \rightarrow L^{p}$ равномерно ораничены при $1 \leqslant p \leqslant \infty$. Для $k<j$ в силу замечания $21.2 Q_{j}=2^{-j r} \sum_{|\alpha|=r} \mathrm{R}_{j}^{(\alpha)} \partial^{\alpha}$. Поэтому $\left\|Q_{j}\left(g_{k}\right)\right\|_{p} \leqslant C \varepsilon_{k} 2^{(r-s) k} 2^{-r j}$. Окончательно,

$$
\left\|Q_{j}(f)\right\|_{p} \leqslant C 2^{-r j} \sum_{k=0}^{j} \varepsilon_{k} 2^{(r-s) k}+C \sum_{k=j+1}^{\infty} \varepsilon_{k} 2^{-j s} \leqslant C^{\prime} \widetilde{\varepsilon}_{j} 2^{-s k},
$$

где $\widetilde{\varepsilon}_{j}:=\sum_{k} \varepsilon_{k} 2^{-(r-s)|j-k|}$ принадлежит $l^{q}$ как свертка $l^{q}$-последовательности с $l^{1}$-последовательностью.

По аналогии с пространствами Соболева определение пространств Бесова можно распространить на неположительные $s$.

ОПРЕДЕЛЕНИЕ 24.1. Пусть $s \leqslant 0,|s|<r \in \mathbb{N}$. Будем говорить, что $f \in B_{p}^{s, q}\left(\mathbb{R}^{n}\right)$, если $P_{0} f \in L^{p}\left(\mathbb{R}^{n}\right)$ и $\left\|Q_{j} f\right\|_{p} \leqslant \varepsilon_{j} 2^{-j s}$, где $\left\{\varepsilon_{j}\right\}_{j=0}^{\infty} \in l^{q}(\mathbb{N})$.

Легко видеть, что при $s^{\prime}=-s, s^{\prime}>0,1 / p+1 / p^{\prime}=1,1 / q+1 / q^{\prime}=1$ двойственность между распределениями $f \in B_{p}^{s, q}\left(\mathbb{R}^{n}\right)$ и пробными функциями $g \in B_{p^{\prime}}^{s^{\prime}}, q^{\prime}\left(\mathbb{R}^{n}\right)$ определяется следуюшим образом:

$$
\langle f, g\rangle=\left\langle P_{0} f, P_{0} g\right\rangle+\sum_{0}^{\infty}\left\langle Q_{j} f, Q_{j} g\right\rangle .
$$

Если $p>1$ и $q>1$, то $B_{p}^{s, q}\left(\mathbb{R}^{n}\right)$ - двойственное пространство к $B_{p^{\prime}}^{s^{\prime}} q^{\prime}\left(\mathbb{R}^{n}\right)$ и его определение не зависит от выбора КМА. Для $p=1$ или $q=1$ независимость определения от КМА проверяется непосредственно.

Используя утверждение 24.1, нетрудно показать, что

$$
\mathbb{C}^{r}\left(\mathbb{R}^{n}\right)=B_{\infty}^{r, \infty}\left(\mathbb{R}^{n}\right)=\left(B_{1}^{-r, 1}\right)^{*}\left(\mathbb{R}^{n}\right)
$$


УТВЕРЖДЕНИЕ 24.2. Пусть $V_{j}, j \in \mathbb{Z},-r$-регулярный кратномасштабный анализ в $L^{2}\left(\mathbb{R}^{n}\right), r>s$. Тогда $f \in \mathbb{C}^{s}\left(\mathbb{R}^{n}\right)$ тогда и только тогда, когда $P_{0} f \in$ $L^{\infty}\left(\mathbb{R}^{n}\right)$ u $\left\|Q_{j} f\right\|_{\infty} \leqslant C 2^{-j s}$ для некоторой константы $C$.

\section{5. Проекторы $P_{j}$ и псевдодифференциальные операторы.}

ОПРЕДЕЛЕНИЕ 25.1. Пусть символ $\sigma(x, \omega)$ является функцией от $x \in \mathbb{R}^{n}$ и от $\omega \in \mathbb{R}^{n}$ (иногда дополнительно требуют, чтобы $\omega \neq 0$ ). Псевдодифференциальньй оператор (ПДО) $T$ определяется посредством формального правила

$$
T\left(e^{i x \cdot \omega}\right)=\sigma(x, \omega) e^{i x \cdot \omega} .
$$

ОПРЕДЕлЕниЕ 25.2. Класс Хермандера $S_{\rho, \delta}^{0}$ определяется условием

$$
\left|\partial_{\omega}^{\alpha} \partial_{x}^{\beta} \sigma(x, \omega)\right| \leqslant C(\alpha, \beta)(1+|\omega|)^{\rho|\beta|-\delta|\alpha|},
$$

где случай $\rho=\delta=1$ исключается из рассмотрения.

Произвольная функция из $S\left(\mathbb{R}^{n}\right)$ равна $f(x)=\frac{1}{(2 \pi)^{n}} \int_{\mathbb{R}^{n}} e^{i x \cdot \omega} \hat{f}(\omega) d \omega$, поэтому для линейного $T$ получаем, что

$$
T f(x)=\frac{1}{(2 \pi)^{n}} \int_{\mathbb{R}^{n}} e^{i x \cdot \omega} \sigma(x, \omega) \hat{f}(\omega) d \omega .
$$

Формула (25.3) имеет смысл для $\sigma(x, \omega) \in L^{\infty}\left(\mathbb{R}^{n} \times \mathbb{R}^{n}\right)$.

Пусть $V_{j}, j \in \mathbb{Z},-r$-регулярный $\mathrm{KMA} \mathrm{в} L^{2}\left(\mathbb{R}^{n}\right), \varphi$ - ортогональная масштабируюшая функция, $P_{j}: L^{2}\left(\mathbb{R}^{n}\right) \rightarrow V_{j}$ - ортогональные проекторы.

УТВЕРЖДЕНИЕ 25.1. Символ проектора $P_{j}$ равен $\sigma\left(2^{j} x, 2^{-j} \omega\right)$, где

$$
\sigma(x, \omega):=\sum_{k \in \mathbb{Z}^{n}} e^{2 \pi i k \cdot x} \widehat{\varphi}(\omega+2 k \pi) \overline{\widehat{\varphi}(\omega)}
$$

ДоКАЗАТЕЛЬСТво. Пусть $\sigma(x, \omega)$ символ $P_{0}$. Легко видеть, что для любого $a \in \mathbb{R}^{n}$ $\sigma(x-a, \omega)$ является символом оператора $R_{a} P_{0} R_{a}^{-1}$, где $R_{a} f(x):=f(x-a)$. Так как оператор $P_{0}$ коммутирует со сдвигами $R_{k}, k \in \mathbb{Z}^{n}$, его символ $\sigma(x, \omega)$ является $\mathbb{Z}^{n}$-периодической функцией по $x$. Заметим, что

$$
\sum_{k \in \mathbb{Z}^{n}} e^{2 \pi i k \cdot x} \widehat{\varphi}(\omega+2 k \pi)=\sum_{l \in \mathbb{Z}^{n}} e^{-i \omega \cdot(x+l)} \varphi(x+l)
$$

так KaK

$$
\begin{aligned}
\int_{[0,1]^{n}} e^{-2 \pi i k \cdot x}\left(\sum_{l \in \mathbb{Z}^{n}} e^{-i \omega \cdot(x+l)} \varphi(x+l)\right) d x \\
=\int_{\mathbb{R}^{n}} e^{-2 \pi i k \cdot x} e^{-i \omega \cdot x} \varphi(x) d x=\widehat{\varphi}(\omega+2 \pi k) .
\end{aligned}
$$


Таким образом, (25.4) переписывается в виде

$$
\sigma(x, \omega)=\left(\sum_{l \in \mathbb{Z}^{n}} e^{-i \omega \cdot(x+l)} \varphi(x+l)\right) \overline{\widehat{\varphi}(\omega)} .
$$

Для доказательства (25.5) воспользуемся тождеством $P_{0}\left(e^{i x \cdot \omega}\right)=\sigma(x, \omega) e^{i x \cdot \omega}$. Так как $E_{0}(x, y):=\sum_{k \in \mathbb{Z}} \varphi(x-k) \overline{\varphi(y-k)}$, то

$$
\begin{aligned}
P_{0}\left(e^{i x \cdot \omega}\right) & =\sum_{k \in \mathbb{Z}} \varphi(x-k) \int_{\mathbb{R}^{n}} \bar{\varphi}(y-k) e^{i y \cdot \omega} d y \\
& =\sum_{k \in \mathbb{Z}} \varphi(x-k) e^{i(k-x) \cdot \omega} \overline{\widehat{\varphi}(\omega)} e^{i x \cdot \omega}=\sigma(x, \omega) e^{i x \cdot \omega} .
\end{aligned}
$$

Так как производные $\varphi$ порядка не выше $r$ быстро убьвают, то

$$
\left|\partial_{\omega}^{\alpha} \partial_{x}^{\beta} \sigma(x, \omega)\right| \leqslant C(\alpha)
$$

для любого $\alpha \in \mathbb{N}^{n}$ и любого $\beta \in \mathbb{N}^{n},|\beta| \leqslant r$.

Исследуем, какие свойства символа $\sigma(x, \omega)$ следуют из теоремы 21.1. Вычислим $P_{0}\left(x^{\alpha}\right)$, используя (25.3). Для этого приблизим $x^{\alpha}$ функциями из класса Шварца. Рассмотрим для любого $\epsilon>0$ функцию $f_{\epsilon}(x)=x^{\alpha} e^{-\epsilon|x|^{2}}$, преобразование Фурье которой равно $i^{|\alpha|} \partial_{\omega}^{\alpha} g_{\epsilon}(\omega)$, где $g_{\epsilon}(\omega):=(\pi / \varepsilon)^{n / 2} e^{-|\omega|^{2} / 4 \varepsilon}$. Тогда

$$
P_{0} f_{\epsilon}(x)=\frac{i^{|\alpha|}}{(2 \pi)^{n}} \int e^{i x \cdot \omega} \sigma(x, \omega) \partial_{\omega}^{\alpha} g_{\epsilon}(\omega) d \omega .
$$

Интегрируя по частям, имеем

$$
P_{0} f_{\epsilon}(x)=\frac{(-i)^{|\alpha|}}{(2 \pi)^{n}} \int \partial_{\omega}^{\alpha}\left(e^{i x \cdot \omega} \sigma(x, \omega)\right) g_{\epsilon}(\omega) d \omega
$$

Так как ядро $E(x, y)$ оператора $P_{0}$ есть $O\left(|x-y|^{-m}\right)$ на бесконечности для любого $m \in \mathbb{N}$, по теореме Лебега получаем, что $P_{0}\left(x^{\alpha}\right)=\lim _{\epsilon \downarrow} P_{0}\left(f_{\epsilon}\right)(x)$. Переходя к пределу в (25.7), имеем

$$
x^{\alpha}=\left.(-i)^{|\alpha|} \partial_{\omega}^{\alpha}\left(e^{i x \cdot \omega} \sigma(x, \omega)\right)\right|_{\omega=0}
$$

Равенство (25.8) вьполнено для любых $\alpha \in \mathbb{N}^{n}$ с $|\alpha| \leqslant r$. Поэтому $\sigma(x, 0)=1$ и $\left.\partial_{\omega}^{\alpha} \sigma(x, \omega)\right|_{\omega=0}=0$ при $1 \leqslant|\alpha| \leqslant r$.

Учитывая $(25.4)$, имеем $\sigma(x, 0)=\sum_{k \in \mathbb{Z}^{n}} e^{2 \pi i k \cdot x} \widehat{\varphi}(0+2 k \pi) \overline{\widehat{\varphi}(0)}=1$, откуда следует, что

$$
\widehat{\varphi}(2 k \pi)=0 \quad \forall k \neq 0 .
$$

По индукции получим, что $\left(\partial^{\alpha} \widehat{\varphi}\right)(2 k \pi)=0 \forall k \neq 0,|\alpha| \leqslant r$.

Так как $\varphi(x) \leqslant C_{m}(1+|x|)^{-m}$ для любого $m \in \mathbb{N}$, то ряд $\sum_{k \in \mathbb{Z}^{n}}|\widehat{\varphi}(\omega+2 k \pi)|^{2}$ и все его производные равномерно сходятся на компактах. Из теоремы 9.1 следует 
УТВЕРЖДЕНИЕ 25.2.

$$
|\widehat{\varphi}(\omega)|^{2}=1+O\left(|\omega|^{2 r+2}\right) \quad \text { npu } \quad|\omega| \rightarrow 0 .
$$

Всегда можно скорректировать $\varphi$ таким образом, чтобы скорректированная функция $\widetilde{\varphi}$ удовлетворяла условиям

$$
\int_{\mathbb{R}^{n}} \widetilde{\varphi}(x) d x=1 \quad \text { и } \int_{\mathbb{R}^{n}} x^{\alpha} \widetilde{\varphi}(x) d x=0
$$

при $1 \leqslant|\alpha| \leqslant r$, сохраняя при этом свойство ортогональности $\{\widetilde{\varphi}(\cdot-k)\}_{k \in \mathbb{Z}}$ и быстрого убывания на бесконечности производных $\partial^{\alpha} \widetilde{\varphi}$ при $|\alpha| \leqslant r$. Для этого умножим сначала $\widehat{\varphi}$ на константу, по модулю равную 1 , для того, чтобы $\widehat{\widetilde{\varphi}}(0)=1$. Далее, пусть $\alpha(\omega)$ - аргумент $\widehat{\varphi}(\omega)$ в окрестности 0 , т.е. $\widehat{\varphi}(\omega)=|\widehat{\varphi}(\omega)| e^{i \alpha(\omega)}$ для $|\omega|<\delta$, где $\alpha(0)=0$ и $\alpha(\omega)$ - бесконечно дифференцируемая функция на $|\omega|<\delta$. Пусть действительная, бесконечно дифференцируемая функция $\beta(\omega)$ является $2 \pi$-периодическим продолжением $\alpha(\omega)$ на $\mathbb{R}^{n}$. Определим $\widehat{\widetilde{\varphi}}(\omega)$ как $e^{-i \beta(\omega)} \widehat{\varphi}(\omega)$. Тогда $\widehat{\widetilde{\varphi}}(\omega)=|\widehat{\varphi}(\omega)|$ в окрестности 0 и вьполнено (25.11).

УТВЕРЖДЕНИЕ 25.3. Всплески $\psi_{1}, \ldots, \psi_{q}, q:=2^{n}-1$, определенные в теореме 19.3, удовлетворяют условиям

$$
\int_{\mathbb{R}^{n}} x^{\alpha} \psi_{l}(x) d x=0
$$

$\partial \Omega_{\text {я }}|\alpha| \leqslant r \quad u 1 \leqslant l \leqslant q$.

ДоКАЗАТЕЛЬСТВо. Пусть $\widehat{\varphi}(\omega)=m_{0}(\omega / 2) \widehat{\varphi}(\omega / 2), \widehat{\psi}_{l}(\omega)=m_{l}(\omega / 2) \widehat{\varphi}(\omega / 2)$, где $m_{l}$ - бесконечно дифференцируемые $2 \pi \mathbb{Z}^{n}$-периодические функции. Аналогично одномерному случаю доказьвается, что $\left|m_{0}(\omega)\right|^{2}+\left|m_{1}(\omega)\right|^{2}+\cdots+\left|m_{q}(\omega)\right|^{2}=1$, откуда следует, что $|\widehat{\varphi}(\omega)|^{2}+\left|\widehat{\psi}_{1}(\omega)\right|^{2}+\cdots+\left|\widehat{\psi}_{q}(\omega)\right|^{2}=|\widehat{\varphi}(\omega / 2)|^{2}$. Из (25.10) следует, что $\widehat{\psi}_{l}(\omega)=O\left(|\omega|^{r+1}\right), 1 \leqslant l \leqslant q$. Так как $\psi_{l}$ быстро убывают на бесконечности, их преобразование Фурье бесконечно дифференцируемо. Значит, $\partial^{\alpha} \psi_{l}(\omega)=0$ при $\omega=0$ и $1 \leqslant l \leqslant q$, откуда следует $(25.12)$.

ПримеР. Применим (25.4) к КМА Мейера (см. раздел 14). Так как supp $\widehat{\varphi}^{M}=$ $[-4 \pi / 3,4 \pi / 3]$, то в $(25.4)$ всего три не равных нулю члена:

$$
\sigma(t, \omega)=e^{-2 \pi i t} \widehat{\varphi}(\omega-2 \pi) \widehat{\varphi}(\omega)+(\widehat{\varphi}(\omega))^{2}+e^{2 \pi i t} \widehat{\varphi}(\omega+2 \pi) \widehat{\varphi}(\omega) .
$$

Пусть $S_{j}$ - оператор свертки, соответствующий мультипликатору $\left(\widehat{\varphi}\left(2^{-j} \omega\right)\right)^{2}$. Обозначим

$$
\eta(\omega):=\widehat{\varphi}(\omega-2 \pi) \widehat{\varphi}(\omega), \quad \theta(\omega):=\widehat{\varphi}(\omega+2 \pi) \widehat{\varphi}(\omega) .
$$

Ясно, что $\operatorname{supp} \eta=[2 \pi / 3,4 \pi / 3], \eta(2 \pi-\omega)=\eta(\omega), \operatorname{supp} \theta=[-4 \pi / 3,-2 \pi / 3]$, $\theta(-2 \pi-\omega)=\theta(\omega)$. Пусть $\Delta_{j}^{+}$и $\Delta_{j}^{-}-$операторы свертки, соответствуюшие мультипликаторам $\eta\left(2^{-j} \omega\right)$ и $\theta\left(2^{-j} \omega\right)$ соответственно. Пусть $M_{j}$ - оператор поточечного умножения на $e^{2 \pi i 2^{j} t}$. Из (25.13) следует, что проекторы КМА Мейера имеют вид

$$
P_{j}=S_{j}+M_{j} \Delta_{j}^{-}+M_{j}^{-1} \Delta_{j}^{+}
$$


26. Всплесковая характеризация пространств Гёльдера $\mathbb{C}^{s}$, Соболева $W_{2}^{s}$ и Бесова $B_{p}^{s, q}$. Всплесковые ряды обладают следуюшими преимушествами:

- всплески, локализованные в области регулярности функции, имеют пренебрежимо маленькие коэффициенты, а всплески, локализованные около особенностей функции, наоборот, имеют большие коэффициенты (эта особенность выражена тем ярче, чем больше нулевых моментов у всплеска);

- всплесковые коэффициенты позволяют вычислять, с точностью до эквивалентности, норму функции в большинстве функциональных пространств.

Пусть $\left\{\psi_{l}\right\}_{l=0}^{2^{n}-1}-$ функции, определенные в теореме 19.3. Многомерный всплесковьй ряд имеет вид

$$
f(x)=\sum_{\lambda \in \Lambda}\left\langle f, \psi_{\lambda}\right\rangle \psi_{\lambda}(x)
$$

где $\Lambda:=\left\{\lambda=(l, j, k): 1 \leqslant l \leqslant 2^{n}-1, k \in \mathbb{Z}^{n}, j \in \mathbb{Z}\right\}, \psi_{\lambda}(x):=2^{n j / 2} \psi_{l}\left(2^{j} x-k\right)$.

В пространствах, отличных от $L^{2}\left(\mathbb{R}^{n}\right)$, использование (26.1) бывает затруднительным. Например, в $L^{\infty}\left(\mathbb{R}^{n}\right)$ для $f \equiv 1(26.1)$ преврашается в $1=0$. Двойственный пример: пусть $f \in D\left(\mathbb{R}^{n}\right)$ и $\int_{\mathbb{R}^{n}} f(x) d x=1$. Так как $\int_{\mathbb{R}^{n}} \psi_{l}(x) d x=0$, то ряд (26.1) не сходится в $L^{1}\left(\mathbb{R}^{n}\right)$.

Поэтому вместо (26.1) удобнее пользоваться рядом по сдвигам масштабирующей функции $\varphi$ и всплескам, сжатым в $2^{j}$ раз с $j \geqslant 0$ :

$$
f(x)=\sum_{k \in \mathbb{Z}^{n}}\left\langle f, \varphi_{0, k}\right\rangle \varphi_{0, k}(x)+\sum_{\lambda \in \cup_{j} \geqslant 0}\left\langle f, \psi_{\lambda}\right\rangle \psi_{\lambda}(x),
$$

где $\Lambda_{j}:=\left\{\lambda=(l, j, k): 1 \leqslant l \leqslant 2^{n}-1, k \in \mathbb{Z}^{n}\right\}$.

Ряд (26.2) хорошо представляет функции из пространств, которые характеризуются некоторым локальным условием и некоторьм глобальным условием роста на бесконечности.

Проиллюстрируем использование (26.1) и (26.2) на примере пространств Соболева $W_{2}^{s}\left(\mathbb{R}^{n}\right)$ (определение 2.6), Гёльдера $\mathbb{C}^{s}\left(\mathbb{R}^{n}\right)$ (определение 2.9 ) и Бесова $B_{p}^{s, q}$ (определение 2.8). Будем рассматривать всплески, полученные из $r$-регулярного КМА. При этом порядок распределения, для которого вычисляются коэффициенты, предполагается строго меншшим $r$. Равенство (26.1) понимается как равенство интегралов от обеих частей равенства, умноженных на функцию из $C^{r}\left(\mathbb{R}^{n}\right)$ с компактным носителем.

Теорема 26.1. Распределение $f$ принадлежит $W_{2}^{s}\left(\mathbb{R}^{n}\right)$ c $s \in(-r, r)$ тогда и только тогда, когда всплесковые коэффициенты $\alpha(\lambda):=\left\langle f, \psi_{\lambda}\right\rangle$ удовлетворяют условиям М

$$
\sum_{j<0} \sum_{\lambda \in \Lambda_{j}}|\alpha(\lambda)|^{2}+\sum_{j \geqslant 0} \sum_{\lambda \in \Lambda_{j}} 4^{j s}|\alpha(\lambda)|^{2}<\infty .
$$

Функиия $f$ принадлежит $\mathbb{C}^{s}\left(\mathbb{R}^{n}\right)$ c $s \in(0, r)$ тогда и только тогда, когда существуют две константы $C_{0}$ и $C_{1}$ такие, что коэффициенты $\beta(k):=\left(f, \varphi_{0, k}\right)$, $k \in \mathbb{Z}^{n}, u \alpha(\lambda):=\left(f, \psi_{\lambda}\right), \lambda \in \Lambda_{j}, j>0$, удовлетворяют условиям $|\beta(k)| \leqslant C_{0}$, $|\alpha(\lambda)| \leqslant C_{1} 2^{-n j / 2} 2^{-j s}$. 
ДокАЗАТЕльство. В случае пространств Соболева теорема следует из теоремы 23.2 и того, что $\left\{\psi_{\lambda}\right\}_{\lambda \in \Lambda_{j}}$ ортонормированный базис в $W_{j}$, поэтому

$$
\left\|Q_{j} f\right\|_{2}^{2}=\sum_{\lambda \in \Lambda_{j}}\left|\left\langle f, \psi_{\lambda}\right\rangle\right|^{2}
$$

Для пространств Гёльдера результат следует из утверждения 24.2 и того, что $\|f\|_{\infty}$ и $\sup _{\lambda \in \Lambda_{0}}\left|\left\langle f, \psi_{\lambda}\right\rangle\right|$ эквивалентны на $W_{0}$. Действительно, $\left|\left\langle f, \psi_{\lambda}\right\rangle\right| \leqslant\left\|\psi_{\lambda}\right\|_{1}\|f\|_{\infty}=$ $C\|f\|_{\infty}$ и $\sup _{\lambda \in \Lambda_{0}}\left|\psi_{\lambda}(x)\right| \leqslant C^{\prime}$ в силу (19.6), откуда $|f(x)| \leqslant C^{\prime} \sup _{\lambda \in \Lambda_{0}}\left|\left\langle f, \psi_{\lambda}\right\rangle\right|$.

ЗАмЕчАниЕ 26.1. Пространство $\mathbb{C}^{s}$ нельзя охарактеризовать, используя только модули коэффициентов ряда (26.1). Это связано с невозможностью охарактеризовать таким способом $L^{\infty}\left(\mathbb{R}^{n}\right)$. Например, при $x \in \mathbb{R}$ модули коэффищиентов всплесков Мейера (см. (14.1)) для $\log |x|$ и для $x /|x|$ асимптотически совпадают. Это связано с тем, что преобразование Гильберта $H$ отображает $x /|x|$ в $\log |x|$, а всплески Мейера $\psi_{j, k}^{M}$ преобразуются в совершенно аналогичные всплески $\widetilde{\psi}_{j, k}^{M}$, где $\widetilde{\psi}^{M}(t):=$ $\left(H \psi^{M}\right)(t)=\frac{1}{2 \pi} \int_{\mathbb{R}} \sin ((t-1 / 2) \xi) \operatorname{sign}(\xi) \sin (\lambda(\xi)) d \xi$.

ЗАмечАниЕ 26.2. Пространство Соболева $W_{2}^{s}$ можно охарактеризовать при помощи ряда (26.2): $f \in W_{2}^{s},-r<s<r$, тогда и только тогда, когда $\sum_{k \in \mathbb{Z}}|\beta(k)|^{2}<\infty$ и $\sum_{j \geqslant 0} \sum_{\lambda \in \Lambda_{j}} 4^{j s}|\alpha(\lambda)|^{2}<\infty$.

Дело в том, что $\sum_{k \in \mathbb{Z}}|\beta(k)|^{2}=\sum_{j<0} \sum_{\lambda \in \Lambda_{j}}|\alpha(\lambda)|^{2}<\infty$, так как $V_{0}-$ прямая сумма пространств $W_{j}, j<0$.

Аналогично лемме 20.1 доказывается

УТВЕРЖДЕНИЕ 26.1. Пусть $p \in[1, \infty]$. Существуют константы $C^{\prime}>C>0$ такие, что для любой конечной сум⿻ы $f(x)=\sum_{\lambda \in \Lambda_{j}} \alpha(\lambda) \psi_{\lambda}(x) \in W_{j}$

$$
C\|f\|_{p} \leqslant 2^{n j\left(\frac{1}{2}-\frac{1}{p}\right)}\left(\sum_{\lambda \in \Lambda_{j}}|\alpha(\lambda)|^{p}\right)^{1 / p} \leqslant C^{\prime}\|f\|_{p} .
$$

Из утверждений 24.1 и 26.1 следует

ТЕОРема 26.2. Функиия

$$
f(x)=\sum_{k \in \mathbb{Z}^{n}} \beta(k) \varphi(x-k)+\sum_{j \geqslant 0} \sum_{\lambda \in \Lambda_{j}} \alpha(\lambda) \psi_{\lambda}(x)
$$

принадлежкт $B_{p}^{s, q}$ тогда и только тогда, когда $\{\beta(k)\}_{k \in \mathbb{Z}^{n}} \in l^{p}\left(\mathbb{Z}^{n}\right) u$

$$
\left(\sum_{\lambda \in \Lambda_{j}}|\alpha(\lambda)|^{p}\right)^{1 / p}=2^{-n j\left(\frac{1}{2}-\frac{1}{p}\right)} 2^{-j s} a_{j}
$$

əде $\left\{a_{j}\right\}_{j \in \mathbb{N}} \in l^{q}(\mathbb{N})$. 
27. Всплесковая характеризация пространств $H^{1}\left(\mathbb{R}^{n}\right)$ и ВМО. Пусть $\left\{\psi_{l}\right\}_{l=0}^{2^{n}-1}$ - всплески, определенные в теореме $19.3, r \geqslant 1, \Lambda:=\{\lambda=(l, j, k): 1 \leqslant l \leqslant$ $\left.2^{n}-1, k \in \mathbb{Z}^{n}, j \in \mathbb{Z}\right\}, \psi_{\lambda}(x):=2^{n j / 2} \psi_{l}\left(2^{j} x-k\right)$.

ТЕОрема 27.1. Всплески $\left\{\psi_{\lambda}\right\}_{\lambda \in \Lambda}$ образуют безусловный базис в вещественном классе Харди $H^{1}\left(\mathbb{R}^{n}\right)$.

СХемА ДОКАЗАТЕЛЬСТВА. Сначала рассмотрим всплески с компактным носителем. Не ограничивая общности, можно считать, что $\int_{[0,1]^{n}}\left|\psi_{l}(x)\right|^{2} d x \neq 0,1 \leqslant l<2^{n}$ (этого всегда можно добиться за счет соответствуюшего сдвига). Тогда сушествуют константы $c>0, \gamma>0$ и кубы $A_{l} \subset[0,1)^{n}$ такие, что $\left|\psi_{l}(x)\right| \geqslant c$, если $x \in A_{l}$, и $\left|A_{l}\right| \geqslant \gamma$

Если $\lambda=(l, j, k), 1 \leqslant l \leqslant 2^{n}-1, k \in \mathbb{Z}^{n}, j \in \mathbb{Z}$, то по определению $Q(\lambda):=$ $Q(j, k):=\left\{x \in \mathbb{R}^{n}: 2^{j} x-k \in[0,1)^{n}\right\}$ и $R(\lambda):=\left\{x \in \mathbb{R}^{n}: 2^{j} x-k \in A_{l}\right\}$.

Пусть $f(x)=\sum_{\lambda \in \Lambda} \alpha(\lambda) \psi_{\lambda}(x)$. Утверждение теоремы следует из того, что $\|f\|_{H^{1}\left(\mathbb{R}^{n}\right)}$ эквивалентна следуюшим величинам:

$$
\begin{gathered}
\left\|\left(\sum_{\lambda \in \Lambda}|\alpha(\lambda)|^{2}\left|\psi_{\lambda}(x)\right|^{2}\right)^{1 / 2}\right\|_{L^{1}\left(\mathbb{R}^{n}\right)} ; \\
\left\|\left(\sum_{\lambda \in \Lambda}|\alpha(\lambda)|^{2}|Q(\lambda)|^{-1} \kappa_{R(\lambda)}(x)\right)^{1 / 2}\right\|_{L^{1}\left(\mathbb{R}^{n}\right)} ; \\
\left\|\left(\sum_{\lambda \in \Lambda}|\alpha(\lambda)|^{2}|Q(\lambda)|^{-1} \kappa_{Q(\lambda)}(x)\right)^{1 / 2}\right\|_{L^{1}\left(\mathbb{R}^{n}\right)}
\end{gathered}
$$

Здесь $|e|$ - мера Лебега множества $e$.

В доказательстве используется атомарное описание $H^{1}\left(\mathbb{R}^{n}\right)$.

ОПРЕДЕЛЕНИЕ 27.1. Атомом в $H^{1}\left(\mathbb{R}^{n}\right)$ назьвают функцию $a(x)$ из $L^{2}\left(\mathbb{R}^{n}\right)$, для которой сушествует шар $B \subset \mathbb{R}^{n}$ такой, что выполнены три свойства

$$
\begin{gathered}
a(x)=0, \quad \text { если } x \notin B ; \\
\|a\|_{2} \leqslant|B|^{-1 / 2} ; \\
\int_{B} a(x) d x=0 .
\end{gathered}
$$

ТЕОРема 27.2 [8]. Функиия $f \in L^{1}\left(\mathbb{R}^{n}\right)$ принадлежит $H^{1}\left(\mathbb{R}^{n}\right)$ тогда и только тогда, когда существует последовательность атомов $a_{j}(x)$ и последовательность чисел $\lambda_{j}$ такие, что

$$
\sum_{0}^{\infty}\left|\lambda_{j}\right|<\infty \quad u \quad f(x)=\sum_{0}^{\infty} \lambda_{j} a_{j}(x) .
$$


Оказьвается возможным сгруппировать члены всплескового ряда

$$
f(x)=\sum_{\lambda \in \Lambda} \alpha(\lambda) \psi_{\lambda}(x)
$$

так, что частные суммы $\sum_{\lambda \in \Lambda(l, m, r)} \alpha(\lambda) \psi_{\lambda}(x)$, где $\{\Lambda(l, m, r)\}$ - дизъюнктное разбиение $\Lambda$, образуют атомарное представление $f$. Множества $\Lambda(l, m, r)$ определяются следующим образом. Пусть

$$
\sigma_{l}(x):=\left(\sum_{Q \in \Omega}|\alpha(l, Q)|^{2}|Q|^{-1} \kappa_{R(l, Q)}(x)\right)^{1 / 2}
$$

где $\Omega$ - совокупность всех двоичных кубов, $\alpha(l, Q)=\alpha(l, j, k)$ для $Q=Q(j, k)$, $R(l, Q):=R(l, j, k)$. Пусть $E(l, m):=\left\{x: \sigma_{l}(x)>2^{m}\right\}, m \in \mathbb{Z}$. Ясно, что

$$
\sum_{m \in \mathbb{Z}} 2^{m}|E(l, m)| \leqslant 2 \int_{\mathbb{R}^{n}} \sigma_{l}(x) d x .
$$

Обозначим через $\Omega(l, m)$ совокупность двоичных кубов $Q$, для которых $|Q \cap E(l, m)| \geqslant$ $\beta|Q|$, где $\beta \in(0, \gamma)$ - фиксированная постоянная. Пусть $\{A(l, m, r)\}_{r}-$ совокупность всех максимальных по включению кубов в $\Omega(l, m)$. Легко видеть, что

$$
\left|\bigcup_{r} A(l, m, r)\right|<\frac{1}{\beta}|E(l, m)| \text {. }
$$

Наконец, $\Lambda(l, m, r)$ состоит из тех $\lambda=(l, j, k) \in \Lambda$, для которых $Q(\lambda) \in \Omega(l, m) \backslash$ $\Omega(l, m+1)$ и $Q(\lambda) \subset A(l, m, r)$. Нетрудно показать, что

$$
\sum_{\lambda \in \Lambda(l, m, r)}|\alpha(\lambda)|^{2} \leqslant \frac{1}{\gamma-\beta} 4^{m+1}|A(l, m, r)| .
$$

Пусть $b(l, m, r):=|A(l, m, r)|^{1 / 2}\left(\sum_{\lambda \in \Lambda(l, m, r)}|\alpha(\lambda)|^{2}\right)^{1 / 2}, \widetilde{\alpha}(\lambda):=(b(l, m, r))^{-1} \alpha(\lambda)$ для $\lambda \in \Lambda(l, m, r)$ и $\widetilde{\alpha}(\lambda):=0$ в противном случае. Из (27.9), (27.10) и (27.8) следует, что для любого $l, 1 \leqslant l \leqslant 2^{n}-1$,

$$
\begin{aligned}
\sum_{m \in \mathbb{Z}} \sum_{r} b(l, m, r) & \leqslant \frac{2}{(\gamma-\beta)^{1 / 2}} \sum_{m \in \mathbb{Z}} 2^{m}\left|\bigcup_{r} A(l, m, r)\right| \\
& \leqslant \frac{2}{\beta(\gamma-\beta)^{1 / 2}} \sum_{m \in \mathbb{Z}} 2^{m}|E(l, m)| \leqslant \frac{4}{\beta(\gamma-\beta)^{1 / 2}}\left\|\sigma_{l}\right\|_{1} .
\end{aligned}
$$

Так как мы пока рассматриваем всплески с компактным носителем, то сушествует $d \in \mathbb{N}$ такое, что $\operatorname{supp} \psi_{\lambda} \subset d Q(\lambda)$, где $d Q$ обозначает куб с тем же центром и в $d$ раз большей, чем у $Q$, длиной стороны. Функци $a(l, m, r, x):=$ $d^{-n / 2} \sum_{\lambda \in \Lambda(l, m, r)} \widetilde{\alpha}(\lambda) \psi_{\lambda}(x)$ являются атомами в $H^{1}\left(\mathbb{R}^{n}\right)$. Действительно, 
$\operatorname{supp} a(l, m, r, x) \subset d Q(l, m, r)$ и $\|a(l, m, r, \cdot)\|_{2} \leqslant d^{-n / 2}\left(\sum_{\lambda \in \Lambda(l, m, r)}|\widetilde{\alpha}(\lambda)|^{2}\right)^{1 / 2} \leqslant$ $|d Q(l, m, r)|^{-1 / 2}$. Поэтому ряд, определяюший $a(l, m, r, x)$, сходится в $L^{1}\left(\mathbb{R}^{n}\right)$, и почленное интегрирование дает последнее свойство атома $\int_{\mathbb{R}^{n}} a(l, m, r, x) d x=0$ в силу (25.12).

Таким образом, доказано, что из конечности (27.2) следует возможность атомарного представления функщии $f$.

Покажем, что из атомарного представления $f$ следует интегрируемость функции

$$
S f(x):=\left(\sum_{\lambda \in \Lambda}\left|\left\langle f, \psi_{\lambda}\right\rangle\right|^{2}\left|\psi_{\lambda}(x)\right|^{2}\right)^{1 / 2} .
$$

Ясно, что для этого достаточно проверить выполнение этого свойства для произвольного атома в $H^{1}\left(\mathbb{R}^{n}\right)$. Пусть $f$ - атом, $B$ - соответствующий шар с центром в $x_{0}$ и радиусом $r>0$. Пусть $C$ - некоторая константа, значение которой будет определено ниже. Разобьем $\mathbb{R}^{n}$ на центральньй шар $\widetilde{B}:=\left\{x:\left|x-x_{0}\right| \leqslant C r\right\}$ и двоичные оболочки $R_{m}:=\left\{x: 2^{m} C r \leqslant\left|x-x_{0}\right|<2^{m+1} C r\right\}, m \in \mathbb{N}$. Тогда

$$
\int_{\mathbb{R}^{n}} S f(x) d x=\int_{\widetilde{B}} S f(x) d x+\sum_{0}^{\infty} \int_{R_{m}} S f(x) d x .
$$

Первый интеграл оценивается при помощи неравенства Коши:

$$
\int_{\widetilde{B}} S f(x) d x \leqslant|\widetilde{B}|^{1 / 2}\|S f\|_{2}=C^{n / 2}|B|^{1 / 2}\|f\|_{2} \leqslant C^{n / 2} .
$$

Для оценки остальных интегралов заметим, что $\left\langle f, \psi_{\lambda}\right\rangle=0$, если $d Q(\lambda)$ не пересекается с $B$. Обозначим через $\Lambda^{m}$ совокупность тех $\lambda$, для которых $d Q(\lambda)$ пересекается как с $B$, так и с $R_{m}$. Если константа $C$ достаточно большая, то существует $c>0$ такое, что из $\lambda \in \Lambda^{m}$ следует неравенство $2^{-j} \geqslant c r 2^{m}$, где $2^{-j}$ - длина стороны куба $Q(\lambda)$. Из регулярности всплесков $\psi_{\lambda}$ и того, что $\int_{\mathbb{R}^{n}} f(x) d x=0$, следует, что $\left|\left\langle f, \psi_{\lambda}\right\rangle\right| \leqslant C 2^{n j / 2} 2^{j} r$. Поэтому при $x \in R_{m}$

$$
S f(x) \leqslant C^{\prime} 2^{-m(n+1)} r^{-n} \text { и } \sum_{0}^{\infty} \int_{R_{m}} S f(x) d x<\infty .
$$

Если всплески не имеют компактного носителя, то вместо атомарной характеризации $H^{1}\left(\mathbb{R}^{n}\right)$ используется молекулярная.

ОПРЕДЕЛЕНИЕ 27.2. Пусть $s>n$. Молекулой с центром в $x_{0}$ и шириной $d>0$ называют функцию $f$, удовлетворяющую трем условиям:

$$
\begin{gathered}
\int_{\mathbb{R}^{n}} f(x) d x=0 \\
\int_{\mathbb{R}^{n}}|f(x)|^{2}(1+|x|)^{s} d x<\infty \\
\left(\int_{\mathbb{R}^{n}}|f(x)|^{2}\left(1+\frac{\left|x-x_{0}\right|}{d}\right)^{s} d x\right)^{1 / 2} \leqslant d^{-n / 2} .
\end{gathered}
$$


Нетрудно проверить, что в теореме 27.2 атомы можно заменить на молекуль.

В случае всплесков с некомпактным носителем ранее определенные функции $a(l, m, r, x):=d^{-n / 2} \sum_{\lambda \in \Lambda(l, m, r)} \widetilde{\alpha}(\lambda) \psi_{\lambda}(x)$, не будучи атомами, являются молекулами.

Пространство ВМО $\left(\mathbb{R}^{n}\right)$ является сопряженным к $H^{1}\left(\mathbb{R}^{n}\right)$.

ТЕОРема 27.3. Если $f(x)$ принадлежит $\mathrm{BMO}\left(\mathbb{R}^{n}\right)$, то всплесковые коэффичиенты $\alpha(\lambda):=\left\langle f, \psi_{\lambda}\right\rangle$ удовлетворяют условию Карлесона

$$
\sum_{Q(\lambda) \subset Q}|\alpha(\lambda)|^{2} \leqslant C|Q|
$$

для любого двоичного куба $Q$.

Обратно, если коэффициенты $\alpha(\lambda), \lambda \in \Lambda$, удовлетворяют (27.12), то ряд $\sum_{\lambda \in \Lambda} \alpha(\lambda) \psi_{\lambda}(x)$ сходится в слабой топологии $\sigma\left(\mathrm{BMO}, H^{1}\right) \kappa$ функиии из ВМО.

ДокАЗАТЕльство. Для упрощения вькладок предполагаем, что всплески $\psi_{l}$, $1 \leqslant l \leqslant 2^{n}-1$, имеют компактньй носитель. Общий случай рассматривается аналогично.

Необходимость. Пусть $\operatorname{supp} \psi_{\lambda} \subset d Q(\lambda)$ ( $d Q$ обозначает куб с тем же центром и в $d$ раз большей, чем у $Q$, длиной стороны). Зафиксируем произвольньй двоичньй куб $Q$. Представим $f \in$ ВМО в виде $f=f_{1}+f_{2}+f_{d Q}$, где $f_{d Q}=\frac{1}{|d Q|} \int_{d Q} f(x) d x$, $f_{1}(x)=f(x)-f_{d Q}$ при $x \in d Q$ и $f_{1}(x)=0$ при $x \notin d Q$. Ясно, что $\left\langle f_{2}, \psi_{\lambda}\right\rangle=0$, если $Q(\lambda) \subset Q$. Учитывая (25.12), получаем, что $\left\langle f, \psi_{\lambda}\right\rangle=\left\langle f_{1}, \psi_{\lambda}\right\rangle$ при $Q(\lambda) \subset Q$. Поэтому

$$
\sum_{Q(\lambda) \subset Q}|\alpha(\lambda)|^{2} \leqslant \sum_{\lambda \in \Lambda}\left|\left\langle f_{1}, \psi_{\lambda}\right\rangle\right|^{2}=\left\|f_{1}\right\|_{2}^{2} \leqslant d^{n}\|f\|_{\mathrm{BMO}}^{2}|Q| .
$$

Достаточность. Пусть вьполнено условие (27.12). Рассмотрим шар $B$ радиуса $r$ с центром в $x_{0}$. Выберем $q \in \mathbb{Z}$ так, чтобы $2^{-q} \leqslant r<2^{-q+1}$. Легко видеть, что при $j \geqslant q$ из того, что $d Q(\lambda) \cap B \neq \varnothing$ и длина стороны $Q(\lambda)$ равна $2^{-j}$, следует, что $Q(\lambda) \subset M B$, где константа $M$ зависит только от $d$. Пусть $\Lambda_{1,1}:=\{\lambda=$ $(l, j, k) \in \Lambda: j \geqslant q, Q(\lambda) \subset M B\}, \Lambda_{1,2}:=\left\{\lambda=(l, j, k) \in \Lambda: j \geqslant q, \lambda \notin \Lambda_{1,1}\right\}$, $f_{1,1}:=\sum_{\lambda \in \Lambda_{1,1}} \alpha(\lambda) \psi_{\lambda}, f_{1,2}:=\sum_{\lambda \in \Lambda_{1,2}} \alpha(\lambda) \psi_{\lambda}$. Тогда функция $f_{1,2}$ равна нулю на $B$, а для $f_{1,1}$ имеем оценку $\left\|f_{1,1}\right\|_{2}^{2} \leqslant \sum_{Q(\lambda) \subset M B}|\alpha(\lambda)|^{2} \leqslant C|B|$.

Рассмотрим $j<q$. Для каждого такого $j$ только $M^{n}$ всплесков $\psi_{\lambda}$ с длиной стороны $Q(\lambda)$, равной $2^{-j}$, не равны нулю тождественно на $B$. Для каждого такого всплеска в силу (19.6) имеем $\left|\psi_{\lambda}(x)-\psi_{\lambda}\left(x_{0}\right)\right| \leqslant C 2^{j} 2^{n j / 2}\left|x-x_{0}\right|$. Из (27.12) следует, что $|\alpha(\lambda)| \leqslant C|Q(\lambda)|^{1 / 2}=C 2^{-n j / 2}$. Из всего сказанного получаем, что при $x \in B$

$$
\begin{aligned}
\mid \sum_{\{\lambda=(l, j, k) \in \Lambda: j \leqslant q\}} \alpha(\lambda) \psi_{\lambda}(x) & -\sum_{\{\lambda=(l, j, k) \in \Lambda: j \leqslant q\}} \alpha(\lambda) \psi_{\lambda}\left(x_{0}\right) \mid \\
& \leqslant C M^{n} \sum_{j<q} 2^{j}\left|x-x_{0}\right|=C M^{n} 2^{q}\left|x-x_{0}\right| \leqslant 2 C M^{n} .
\end{aligned}
$$


28. Всплесковая характеризация пространств $L^{p}\left(\mathbb{R}^{n}\right)$ и $W_{p}^{s}\left(\mathbb{R}^{n}\right)$. Пусть $\psi_{\lambda}, \lambda \in \Lambda,-$ всплески, построенные на основе $r$-регулярного KМА при помоши теоремы 19.3, $r \geqslant 1$. Используемые в дальнейшем обозначения определены в предыдушем разделе.

ТеОрема 28.1. Пусть $1<p<\infty$. Всплесковый ряд $\sum_{\lambda \in \Lambda} \alpha(\lambda) \psi_{\lambda}(x)$ принадлежит $L^{p}\left(\mathbb{R}^{n}\right)$ тогда и только тогда, когда

$$
\left\|\left(\sum_{\lambda \in \Lambda}|\alpha(\lambda)|^{2}\left|\psi_{\lambda}(x)\right|^{2}\right)^{1 / 2}\right\|_{p}<\infty .
$$

Кроме того, норма

$$
\left\|\left(\sum_{\lambda \in \Lambda}|\alpha(\lambda)|^{2}\left|\psi_{\lambda}(x)\right|^{2}\right)^{1 / 2}\right\|_{p}
$$

әквивалентна норме

$$
\left\|\left(\sum_{\lambda \in \Lambda}|\alpha(\lambda)|^{2}|Q(\lambda)|^{-1} \kappa_{Q(\lambda)}(x)\right)^{1 / 2}\right\|_{p}
$$

ДокАЗАТЕльство. Пусть $\epsilon:=\{\epsilon(\lambda)= \pm 1\}_{\lambda \in \Lambda}-$ произвольная расстановка знаков. Определим линейный оператор $T_{\epsilon}: L^{2}\left(\mathbb{R}^{n}\right) \mapsto L^{2}\left(\mathbb{R}^{n}\right)$, полагая $T_{\epsilon} \psi_{\lambda}=\epsilon(\lambda) \psi_{\lambda}$. Легко видеть, что операторы $T_{\epsilon}$ являются операторами Кальдерона-Зигмунда (определение 2.5) с ядрами

$$
K_{\epsilon}(x, y)=\sum_{\lambda \in \Lambda} \epsilon(\lambda) \alpha(\lambda) \psi_{\lambda}(x) \bar{\psi}_{\lambda}(y)
$$

удовлетворяюшими в силу (19.11) оценкам $\left|K_{\epsilon}(x, y)\right| \leqslant C|x-y|^{-n}$ и

$$
\left|\frac{\partial K_{\epsilon}}{\partial x_{j}}\right|+\left|\frac{\partial K_{\epsilon}}{\partial y_{j}}\right| \leqslant C|x-y|^{-n-1}
$$

равномерно по $\epsilon$. В силу теоремы 2.1 операторы $T_{\epsilon}$ равномерно ограничены в $L^{p}\left(\mathbb{R}^{n}\right)$. Теперь первое утверждение теоремы следует из неравенств Хинчина.

Второе утверждение следует из того, что линейный оператор $U: L^{2}\left(\mathbb{R}^{n}\right) \mapsto L^{2}\left(\mathbb{R}^{n}\right)$, определяемый равенствами $U\left(\psi_{l}^{H}\right)=\psi_{l}, 1 \leqslant l<2^{n}$, где $\psi_{l}^{H}$ - многомерные всплески Хаара, является изоморфизмом в $L^{p}\left(\mathbb{R}^{n}\right)$. Действительно, $U$ - изоморфизм двоичного $H^{1}\left(\mathbb{R}^{n}\right)$ и обычного $H^{1}\left(\mathbb{R}^{n}\right)$ (двоичное $H^{1}\left(\mathbb{R}^{n}\right)$ состоит из функций, допускающих атомарное представление по атомам с носителями, равными двоичным кубам, см. теорему 27.1). Очевидно, что $U$ - изометрия в $L^{2}\left(\mathbb{R}^{n}\right)$. Остается воспользоваться интерполящией между $H^{1}$ и $L^{2}$ (см. [31]). 
Теорема 28.2. Пусть $1<p<\infty u 0 \leqslant s<r$. Всплесковий ряд $\sum_{\lambda \in \Lambda} \alpha(\lambda) \psi_{\lambda}(x)$ принадлежит $W_{p}^{s}\left(\mathbb{R}^{n}\right)$ тогда и только тогда, когда

$$
\left(\sum_{\lambda \in \Lambda}|\alpha(\lambda)|^{2}\left(1+4^{j s}\right) 2^{n j} \kappa_{Q(\lambda)}(x)\right)^{1 / 2} \in L^{p}\left(\mathbb{R}^{n}\right) .
$$

Если $-r<s \leqslant 0$, то всплесковый ряд принадлежит $W_{p}^{s}\left(\mathbb{R}^{n}\right)$ тогда и только тогда, когда

$$
\left(\sum_{\lambda \in \Lambda}|\alpha(\lambda)|^{2}\left(1+4^{-j s}\right)^{-1} 2^{n j} \kappa_{Q(\lambda)}(x)\right)^{1 / 2} \in L^{p}\left(\mathbb{R}^{n}\right) .
$$

СХема ДокАЗАТЕЛЬСтвА. Сначала рассмотрим всплески Мейера. Для них преобразование Фурье $\widehat{\psi}_{l}$ равно нулю в окрестности нуля и имеет компактньй носитель. Поэтому для любого $\gamma \in \mathbb{R}$ можно определить функции $\psi_{l}^{\gamma}:=(-\Delta)^{\gamma / 2} \psi_{l}$, где $\Delta=\frac{\partial^{2}}{\partial x_{1}^{2}}+\frac{\partial^{2}}{\partial x_{2}^{2}}+\cdots+\frac{\partial^{2}}{\partial x_{n}^{2}}$. Ясно, что $\widehat{\psi}_{l}^{\gamma}(\xi)=|\xi|^{\gamma} \widehat{\psi}_{l}(\xi)$. Определим $\psi_{\lambda}^{\gamma}(x):=2^{n j / 2} \psi_{l}^{\gamma}\left(2^{j} x-k\right)$, где $\lambda=(l, j, k)$. Рассмотрим линейные операторы $D_{\gamma}$, определяемые формулами $D_{\gamma}\left(\psi_{\lambda}\right)=\psi_{\lambda}^{\gamma}$. Для любого $\gamma \in \mathbb{R}$ операторы $D_{\gamma}$ являются изоморфизмами в $L^{p}\left(\mathbb{R}^{n}\right)$. Дело в том, что $\psi_{\lambda}^{\gamma}, \lambda \in \Lambda$, образуют базис Рисса в $L^{2}\left(\mathbb{R}^{n}\right)$, откуда следует, что $D_{\gamma}$ - изоморфизм в $L^{2}\left(\mathbb{R}^{n}\right)$. Кроме того, ядро оператора $D_{\gamma}$ равно $\sum_{\lambda \in \Lambda} \psi_{\lambda}^{\gamma}(x) \bar{\psi}_{\lambda}(y)$ и удовлетворяет требованиям определения 2.5. Значит, по теореме $2.1 D_{\gamma}$ - непрерьвньй оператор в $L^{p}\left(\mathbb{R}^{n}\right)$. Обратньй оператор исследуется аналогично.

Норма $f$ в пространстве $W_{p}^{s}\left(\mathbb{R}^{n}\right)$ эквивалентна $\|f\|_{p}+\left\|(-\Delta)^{s} / 2 f\right\|_{p}$. Поэтому для оценки $W_{p}^{s}\left(\mathbb{R}^{n}\right)$-нормы ряда $\sum_{\lambda \in \Lambda} \alpha(\lambda) \psi_{\lambda}(x)$ необходимо вычислить $L^{p}\left(\mathbb{R}^{n}\right)$-норму $(-\Delta)^{s / 2} \sum_{\lambda \in \Lambda} \alpha(\lambda) \psi_{\lambda}(x)=\sum_{\lambda \in \Lambda} \alpha(\lambda) 2^{j s} \psi_{\lambda}^{s}(x)$. Остается использовать изоморфизм $D_{s}$ в $L^{p}\left(\mathbb{R}^{n}\right)$ и теорему 28.1 .

В обшем случае $r$-регулярного КМА доказьвается, что функции $(-\Delta)^{-s / 2} \psi_{l}$, $1 \leqslant l<2^{n}$, имеют нулевое среднее и вместе со всеми производньми порядка $\leqslant r$ имеют порядок $O\left(|x|^{-n-r+s}\right)$ на бесконечности. Аналогично, функции $(-\Delta)^{s / 2} \psi_{l}$, $1 \leqslant l<2^{n}$, принадлежат классу Гёльдера $H^{r-s}$, убьвают на бесконечности как $O\left(|x|^{-n-r+s}\right)$ и имеют нулевое среднее. Эти свойства позволяют повторить рассуждения, использованные для всплесков Мейера.

Утверждение теоремы для $s<0$ получается по двойственности.

СлеДСтвиЕ 28.1. Ряд

$$
f(x)=\sum_{k \in \mathbb{Z}^{n}} \beta(k) \varphi(x-k)+\sum_{\{\lambda=(l, j, k) \in \Lambda, j \geqslant 0\}} \alpha(\lambda) \psi_{\lambda}(x)
$$

принадлежит $W_{p}^{s}\left(\mathbb{R}^{n}\right), 1<p<\infty,|s|<r$, тогда и только тогда, когда $\{\beta(k)\}_{k \in \mathbb{Z}^{n}} \in l^{p}\left(\mathbb{Z}^{n}\right) u$

$$
\left(\sum_{\{\lambda=(l, j, k) \in \Lambda, j \geqslant 0\}} 2^{n j} 4^{j s}|\alpha(\lambda)|^{2} \kappa_{Q(\lambda)}(x)\right)^{1 / 2} \in L^{p}\left(\mathbb{R}^{n}\right) .
$$


29. Периодические всплески. На основе всплескового базиса на прямой можно построить всплесковьй базис на отрезке $[0,1]$.

Пусть $\left\{V_{j}\right\}_{j \in \mathbb{Z}}-r$-регулярньй KMA в $L_{2}(\mathbb{R}), r \geqslant 1, V_{j}^{\infty}$ - замыкание $V_{j}$ в слабой топологии $\sigma\left(L^{\infty}, L^{1}\right)$. Пусть $T_{j}$ - подпространство функщий из $V_{j}^{\infty}$, имеюших период 1.

Лемма 29.1. Если $j \leqslant 0$, то $T_{j}$ совпадают и состоят только из постоянных функций. Если $j>0$, то размерность $T_{j}$ равна $2^{j}$.

ДокАЗАТЕЛЬство. Заметим, что константы принадлежат всем $V_{j}$, так как

$$
\sum_{k \in \mathbb{Z}} \varphi(t-k) \equiv 1
$$

Последнее следует из (25.9), так как

$$
\int_{-1}^{1} \sum_{k \in \mathbb{Z}} \varphi(t-k) e^{-2 \pi l t} d t=\int_{\mathbb{R}} \varphi(t) e^{-2 \pi l t} d t=\widehat{\varphi}(2 \pi l)=\delta_{l, 0}
$$

Так как $V_{j}^{\infty} \subset V_{j+1}^{\infty}$, то первое утверждение леммы будет доказано, если любая функция $f \in T_{0}$ является константой. Действительно, если $f$ 1-периодична, то последовательность коэффициентов $c_{k}:=\int_{\mathbb{R}} f(t) \overline{\varphi(t-k)} d t$ постоянна, а значит, и $f(t)=$ $\sum_{k \in \mathbb{Z}} c_{k} \varphi(t-k)$ равна константе.

Пусть $j>0$. Тогда любая функция $f \in T_{j}$ имеет вид $f(t)=\sum_{k=-\infty}^{\infty} c_{k} \varphi\left(2^{j} t-k\right)$, где $c_{k}:=2^{j} \int_{\mathbb{R}} f(t) \overline{\varphi\left(2^{j} t-k\right)} d t$. Из периодичности $f$ следует, что $c_{k+2^{j}}=c_{k}$. Очевидно, что верно и обратное. Таким образом, размерность $T_{j}$ равна $2^{j}$.

ЛЕмма 29.2. Обвединение $T_{j}, j \geqslant 0$, плотно в банаховом пространстве непрерывных на действительной оси периодических с периодом 1 функций.

ДокАЗАТЕЛьство. Ортогональный проектор $P_{j}: L^{2}(\mathbb{R}) \rightarrow V_{j}$ определяется формулами

$$
P_{j} f(t)=\int_{\mathbb{R}} E_{j}(t, s) f(s) d s
$$

где $E_{j}(t, s):=2^{j} E\left(2^{j} t, 2^{j} s\right)$ и $E(t, s):=\sum_{k=-\infty}^{\infty} \varphi(t-k) \varphi(s-k)$. Если $f-$ ограничена и равномерно непрерьвна на $\mathbb{R}$, то $\left\|f-P_{j}(f)\right\|_{\infty} \rightarrow 0$ при $j \rightarrow \infty$ (см. лемму 22.1). Остается заметить, что $P_{j}(f) \in T_{j}$ для любого $j \in \mathbb{N}$, если $f$ периодична с периодом 1.

ОПРЕДЕлЕнИЕ 29.1. Вложенная последовательность $\left\{T_{j}\right\}_{j \in \mathbb{N}}$, определенная выше, называется $r$-регулярньм $\mathrm{KMA} \mathrm{в} L^{2}(\mathbb{T})$, где $\mathbb{T}$ - отрезок $[0,1]$ с отождествленными концами.

Пусть $\varphi_{j}^{\text {per }}(t):=2^{j / 2} \sum_{k \in \mathbb{Z}} \varphi\left(2^{j}(t-k)\right)$.

Лемма 29.3. Для любого $j \in \mathbb{N}$ функиии $\varphi_{j}^{\mathrm{per}}\left(t-k 2^{-j}\right), 0 \leqslant k<2^{j}$, образуют ортонормированный базис в $T_{j}$. 
ДоказАТЕльство. Так как размерность $T_{j}$ равна $2^{j}$, то достаточно проверить ортонормированность функций $\varphi_{j}^{\text {per }}\left(t-k 2^{-j}\right), 0 \leqslant k<2^{j}$, в $L^{2}[0,1]$. Для этого необходимо вычислить

$$
2^{j} \sum_{k \in \mathbb{Z}} \sum_{l \in \mathbb{Z}} \int_{0}^{1} \varphi\left(2^{j} t-2^{j} k-m\right) \overline{\varphi\left(2^{j} t-2^{j} l-m^{\prime}\right)} d t .
$$

Сделаем замену переменных $t-k=2^{-j} t^{\prime}$, где $0 \leqslant t<1, k \in \mathbb{Z}$. Она приводит к выражению

$$
\sum_{q \in \mathbb{Z}} \int_{-\infty}^{\infty} \varphi\left(t^{\prime}-m\right) \overline{\varphi\left(t-2^{j} q-m^{\prime}\right)} d t .
$$

Если $0 \leqslant m<2^{j}, 0 \leqslant m^{\prime}<2^{j}$ и $m \neq m^{\prime}$, то каждый из интегралов равен нулю. Если $m=m^{\prime}$, то единственным не равным нулю интегралом является интеграл при $q=0$, которьй равен 1.

Пусть $U_{j}$ обозначает ортогональное дополнение $T_{j}$ до $T_{j+1}$ и пусть

$$
\psi_{j}^{\text {пер }}(t):=2^{j / 2} \sum_{k \in \mathbb{Z}} \psi\left(2^{j}(t-k)\right) .
$$

ЛЕмма 29.4. Для любого $j \in \mathbb{N}$ функиии $\psi_{j}^{\text {пер }}\left(t-k 2^{-j}\right), 0 \leqslant k<2^{j}$, образуют ортонормированный базис в $U_{j}$.

ДоКАЗАТЕЛЬСТВо аналогично доказательству леммы 29.3. Легко видеть, что функции $\psi_{j}^{\text {пер }}\left(t-k 2^{-j}\right)$ принадлежат $T_{j+1}$ и ортогональны к $T_{j}$. Кроме того, функции $\psi_{j}^{\text {пер }}\left(t-k 2^{-j}\right), 0 \leqslant k<2^{j}$, попарно ортогональны. Остается заметить, что размерность $U_{j}$ равна $2^{j}$, так как размерность $T_{j}$ равна $2^{j}$, а размерность $T_{j+1}$ равна $2^{j+1}$.

Таким образом,

$$
L^{2}(\mathbb{T})=T_{0} \oplus U_{0} \oplus U_{1} \oplus U_{2} \oplus \cdots,
$$

откуда следует, что постоянная функция 1 вместе с последовательностью функций $\left\{\psi_{j}^{\text {пер }}\left(t-k 2^{-j}\right)\right\}_{0 \leqslant k<2^{j}, j \in \mathbb{N}}$ образуют ОНБ в $L^{2}(\mathbb{T})$. Занумеруем эту последовательность лексикографически: $g_{0}(t) \equiv 1$; если $m=2^{j}+k, 0 \leqslant k<2^{j}$, то $g_{m}(t):=\psi_{j}^{\text {пер }}\left(t-k 2^{-j}\right)$

ТЕОРЕМА 29.1. Пусть $\left\{V_{j}\right\}_{j \in \mathbb{Z}}-r$-регулярный кратномасштабный анализ в $L^{2}(\mathbb{R}), r \geqslant 1$. Тогда последовательность $\left\{g_{m}\right\}_{m \in \mathbb{N}}$ является базисом Шаудера в $C(\mathbb{T}) \quad$ и $L^{1}(\mathbb{T})$. Она также является базисом Шаудера и в $C^{k}(\mathbb{T})$ при $0 \leqslant k<r$. Та же последовательность $\left\{g_{m}\right\}_{m \in \mathbb{N}}$ является безусловным базисом в пространстве Гёльдера $\mathbb{C}^{\alpha}$ при $0<\alpha<1$, в классе Зигмунда $\Lambda_{\star}=\mathbb{C}^{1}$ при $r \geqslant 2$, в пространствах $L^{p}$ c $1<p<\infty$, в пространстве Харди $H^{1}(\mathbb{T})$ и в его двойственном пространстве $\mathrm{BMO}(\mathbb{T})$.

ЗАмечАНИЕ 29.1. Отметим, что тригонометрическая система не является базисом Шаудера в $C(\mathbb{T})$ и в $L^{1}(\mathbb{T})$. В пространствах $L^{p}$ при $1<p<\infty$ она образует базис Шаудера, но не безусловный. 
ДоказАТельство. Так как $\bigcup T_{j}$ плотно в $C(\mathbb{T})$ (см. лемму 29.2), то для базисности $\left\{g_{m}\right\}_{m \in \mathbb{N}}$ в $C(\mathbb{T})$ достаточно доказать, что $\left\|\sum_{m=0}^{l}\left\langle f, g_{m}\right\rangle g_{m}\right\|_{\infty} \leqslant C\|f\|_{\infty}$ для любого $l \in \mathbb{N}$. Продолжим функцию $f \in C(\mathbb{T})$ по периодичности на всю прямую $\mathbb{R}$. Тогда $\sum_{m=0}^{2^{j}}\left\langle f, g_{m}\right\rangle g_{m}=P_{j} f$, причем операторы $P_{j}$ равномерно ограничены на $L^{\infty}(\mathbb{R})$, так как $\left\|P_{j}\right\|_{\infty}=\left\|P_{0}\right\|_{\infty}$. Пусть $2^{j} \leqslant l<2^{j+1}$. Заметим, что

$$
\left\|\sum_{m=2^{j}}^{l}\left\langle f, g_{m}\right\rangle g_{m}\right\|_{\infty} \leqslant\|f\|_{\infty}\left(\sup _{2^{j} \leqslant m<2^{j+1}}\left\|g_{m}\right\|_{1}\right)\left\|_{2^{j} \leqslant m<2^{j+1}}\left|g_{m}\right|\right\|_{\infty} .
$$

Легко видеть, что $\left\|g_{m}\right\|_{L^{1}(\mathbb{T})}=2^{-j / 2}\|\psi\|_{L^{1}(\mathbb{R})}$ при $2^{j} \leqslant m<2^{j+1}$. Кроме того,

$$
\begin{aligned}
\left\|\sum_{2^{j} \leqslant m<2^{j+1}}\left|g_{m}\right|\right\|_{\infty} & =\left\|\sum_{k \in \mathbb{Z}}\left|2^{j / 2} \psi\left(2^{j} \cdot-k\right)\right|\right\|_{\infty} \\
& =2^{j / 2}\left\|\sum_{k \in \mathbb{Z}}|\psi(\cdot-k)|\right\|_{\infty}=C 2^{j / 2} .
\end{aligned}
$$

Поэтому $\left\|\sum_{m=2^{j}}^{l}\left\langle f, g_{m}\right\rangle g_{m}\right\|_{\infty} \leqslant C\|f\|_{\infty}$.

Случай $C^{k}(\mathbb{T})$ разбирается аналогично с использованием теоремы 21.2.

Базисность в $L^{1}(\mathbb{T})$ получается по двойственности.

Из теоремы 26.1 следует, что

$$
\sum_{m=0}^{\infty} \alpha_{m} g_{m} \in \mathbb{C}^{s}(\mathbb{T}) \Longleftrightarrow \alpha_{m}=O\left(m^{-s-1 / 2}\right)
$$

из чего следует безусловная базисность в $\mathbb{C}^{s}(\mathbb{T})$.

Результат для пространств $L^{p}(\mathbb{T}), H^{1}(\mathbb{T}), \mathrm{BMO}(\mathbb{T})$ следует из теорем 28.1, 27.1 и 27.3 .

Сравним всплесковые ряды и ряды Фурье. Оказывается, что полные всплесковые ряды, имеюшие много больших коэффициентов, представляют собой патологические функции, тогда как всплесковые ряды хороших функций имеют мало больших коэффициентов. Это прямо противоположно ситуации с обычными рядами Фурье: для хороших функций они полные, а для патологических - лакунарные. Это объясняется тем, что всплесковый анализ носит локальньй характер. Большие коэффициенты имеют всплески, локализованные вблизи особенностей анализируемой функции. Вне особенностей анализируемая функция является бесконечно дифференщируемой, и соответствуюшие всплесковые коэффициенты являются пренебрежимо малыми за счет свойства (25.12).

Рассмотрим функцию $|\sin \pi t|^{-\alpha}$, где $0<\alpha<1$. Ее коэффициенты Фурье $c_{k}$, $k \in \mathbb{Z}$, имеют следуюшую ассимптотику $c_{k}=\gamma(\alpha)|k|^{-1+\alpha}+O\left(k^{-3+\alpha}\right)$, где $\gamma(\alpha) \neq 0$ (см. $\left[10\right.$, гл. 5].) Таким образом, особенность в 0 функщии $|\sin \pi t|^{-\alpha}$ влияет на все коэффициенты Фурье. Всплесковые коэффициенты подвергаются влиянию особенности, только если интервал $I_{m}:=\left[k 2^{-j},(k+1) 2^{-j}\right], m=2^{j}+k, 0 \leqslant k<2^{j}$, 


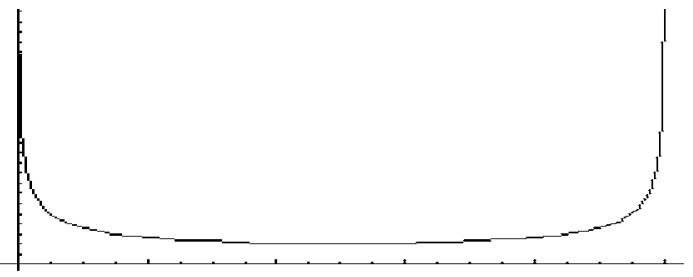

Рис. 24. График $|\sin \pi t|^{-1 / 2}$

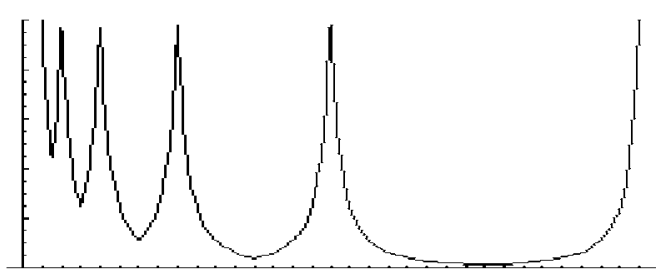

Рис. 25. Оценка модулей всплесковых коэффициентов

определяющий локализацию всплеска $g_{m}(t)$, находится близко к особенности. Более точно, для периодических всплесков, построенных на основе всплесков Мейера, имеем следующую оценку: если $2^{j} \leqslant m<2^{j+1}$ и $l:=\min \left(m-2^{j}, 2^{j+1}-m\right)$, то $\left|\alpha_{m}\right| \leqslant C_{N} 2^{j(\alpha-1 / 2)} /(1+l)^{N}$ для любого натурального $N$. Таким образом, большими будут только коэффициенты с номерами, близкими к $2^{j}, j \in \mathbb{N}$.

Рассмотрим патологические функции. Пусть $q>1, \lambda_{1}>0, \lambda_{k+1}>q \lambda_{k}, k \in \mathbb{N}$, $\sum_{0}^{\infty}\left|\alpha_{k}\right|<\infty$, но $\alpha_{k} \lambda_{k}$ не стремится к 0. Известно [10, гл. 2], что при этих предположениях ряд $\sum_{0}^{\infty} \alpha_{k} \cos \lambda_{k} t$ определяет непрерьвную нигде не дифференщируемую функцию.

Для всплесковых рядов имеем следуюший результат.

ТЕОРема 29.2. Пусть $\left\{g_{m}\right\}_{m \in \mathbb{N}}-$ периодические всплески, полученные из $r$-регулярного кратномасштабного анализа с $r \geqslant 2$. Пусть $f(t)=\sum_{0}^{\infty} \alpha_{m} g_{m}(t)-$ непрерывная функиия, дифференцируемая в точке $t_{0}$. Тогда для любого фиксированного $q \geqslant 1 \alpha_{m}=o\left(m^{-3 / 2}\right)$ при $m$, стремящемся $\kappa$ бесконечности и таком, что интерваль $q I_{m}$ содержат точку $t_{0}$. Здесь $I_{m}:=\left[k 2^{-j},(k+1) 2^{-j}\right]$ при $m=2^{j}+k, 0 \leqslant k<2^{j}$, a $q I_{m}$ - интервал с тем ж⿻е, что и у $I_{m}$, иентром и длиной $q / 2^{j}$.

ДокАЗАТЕльство. Результат легко следует из (19.11) и (25.12).

Из теорем 29.1 и 29.2 следует

СлЕДСТВИЕ 29.1. Пусть последовательность чисел $\alpha_{m}, m \geqslant 1$, удовлетворяет неравенствам $C_{1} m^{-3 / 2} \leqslant\left|\alpha_{m}\right| \leqslant C_{2} m^{-3 / 2}$ для двух констант $C_{2} \geqslant C_{1}>0$. Тогда функиия $f(t)=\sum_{0}^{\infty} \alpha_{m} g_{m}(t)$ принадлежсит классу Зигмунда $\Lambda_{\star}=\mathbb{C}^{1}$, но нигде не дифферениируема.

Функции Вейерштрасса являются частньм случаем следствия 29.1. 
Пусть $\psi^{\text {пер, }} M(t)$ - периодический всплеск Мейера. Прямые вычисления показывают, что $\sum_{-\infty}^{\infty} \psi^{M}(t-k)=-\sqrt{2} \cos 2 \pi t$. Поэтому функция

$$
f(t)=\sum_{j \in \mathbb{N}} \sum_{0}^{2^{j}-1} \alpha(j, k) \psi_{j}^{\text {пер }, M}\left(t-\frac{k}{2^{j}}\right)
$$

с коэффищиентами $\alpha(j, k)=\alpha(j)$, не зависящими от $k$, равна

$$
f(t)=-\sqrt{2} \sum_{0}^{\infty} 2^{j / 2} \alpha(j) \cos \left(2 \pi 2^{j} t\right) .
$$

Таким образом, полный всплесковьй ряд совпадает с лакунарньм рядом Фурье.

\section{СПИСОК ЛИТЕРАТУРЫ}

[1] Grossman A., Morlet J. Decomposition of Hardy functions into square integrable wavelets of constant shape // SIAM J. Math. Anal. 1984. V. 15. P. 723-736.

[2] Meyer Y. Ondelettes et operateurs. Paris: Hermann, 1990.

[3] Daubechies I. Ten lectures on wavelets // CBMS-NSF Regional Conference Series in Applied Mathematics. V. 61. Philadelphia: SIAM, 1992.

[4] Chui C. K. An Introduction to Wavelets. New York: Academic Press, 1992.

[5] Астафььева Н. М. Вейвлет-анализ: основы теории и примеры применения // УФН. 1996. T. 166. №11. C. 1145-1170.

[6] Новиков И.Я., Стечкин С. Б. Основные конструкции всплесков // Фундам. и прикл. матем. 1997. Т. 3. №4. С. 999-1028.

[7] Лузин Н. Н. Sur une propriété des fonctions à carre sommable // Bull. Calcutta Math. Soc. 1930. T. 20. C. 139-154.

[8] Coifman R. R., Weiss G. Extentions of Hardy spaces and their use in analysis // Bull. Amer. Math. Soc. 1977. V. 83. P. 569-645.

[9] Стейн И. Сингулярные интегралы и дифференциальные свойства функций. М.: Мир, 1973.

[10] Зигмунд А. Тригонометрические ряды. Т. 1, 2. М.: Мир, 1965.

[11] Calderon A. P. Commutators of singular integral operators // Proc. Nat. Acad. Sci. USA. 1965. V. 53. P. 1092-1099.

[12] Calderon A.P. Intermediate spaces and interpolation, the complex method // Studia Math. 1964. V. 24. P. 113-190.

[13] Gabor D. Theory of communication // J. Inst. Electr. Engrg. (London). 1946. V. 93. P. $429-457$.

[14] Ландау Л.Д., Лифшиц Е. М. Квантовая механика. М.: Наука, 1974.

[15] Лукашенко Т. П. Всплески на топологических группах // Докл. АН. 1993. Т. 332. № 1. C. $15-17$.

[16] Haar A. Zur theorie der orthogonalen functionensysteme // Math. Ann. 1910. V. 69. P. 331-371.

[17] Mallat S. Multiresolution approximation and wavelets // Trans. Amer. Math. Soc. 1989. V. 315. P. 69-88.

[18] Boor C. de, DeVore R., Ron A. On the construction of multivariate (pre)wavelets // Constructive Approximation. 1993. V. 9. № 2-3. P. 123-166.

[19] Whittaker J. T. Interpolatory function theory. Cambridge: Cambridge Univ. Press, 1935.

[20] Meyer Y. Principe d'incertitude, bases hilbertiennes et algebres d'operateurs // Semin. Bourbaki. 1985-86. №662.

[21] Battle G. A block spin construction of ondelettes, Part II: QFT connection // Commun. Math. Phys. 1988. V. 114. P. 93-102. 
[22] Lemarie P. G. Ondelettes à localisation exponentielle // J. Math. Pures Appl. 1988. V. 67. № 3. P. 227-236.

[23] Полиа Г., Сеге Г. Задачи и теоремы из анализа. Ч. 2. М.: Наука, 1978.

[24] Schoenberg I. J. Contributions to the problem of approximation of equidistant data by analytic functions. Parts A, B // Quart. Appl. Math. 1946. V. 4. P. 45-99, 112-141.

[25] Stromberg J. O. A modified Franklin system and higher order spline systems on $\mathbb{R}^{n}$ as unconditional bases for Hardy spaces // Conf. in honor of A. Zygmund, Vol. II. Belmont, CA: Wadsworth, 1983. P. 475-493.

[26] Daubechies I. Orthonormal basis of compactly supported wavelets // Commun. Pure Appl. Math. 1988. V. 46. P. 909-996.

[27] Smith M.J.T., Barnwell T.P. Exact reconstruction techniques for tree-structured subband coders // IEEE Trans. Acoust. Speech Signal Process. 1986. V. 34. P. 434-441.

[28] Mintzer F. Filters for distortion-free two-band multirate filter banks // IEEE Trans. Acoust. Speech Signal Process. 1985. V. 33. P. 626-630.

[29] Vetterli M. Filter banks allowing perfect reconstruction // Signal Process. 1986. V. 10. P. 219-244.

[30] Gröchenig K. Analyse multiéchelle et bases d'ondelettes // C. R. Acad. Sci. Paris. Sér. I. 1987. V. 305. P. 13-17.

[31] Fefferman C., Stein E. M. $H^{p}$ spaces of several variables // Acta Math. 1972. V. 129. P. 137-193.

Воронежский государственный университет;

Математический институт им. В. А. Стеклова РАН

Поступила в редакцию 28.07.1998 